NISTIR 7671

\title{
Mathematical and Computational Sciences Division
}

\section{Summary of Activities for Fiscal Year 2009}
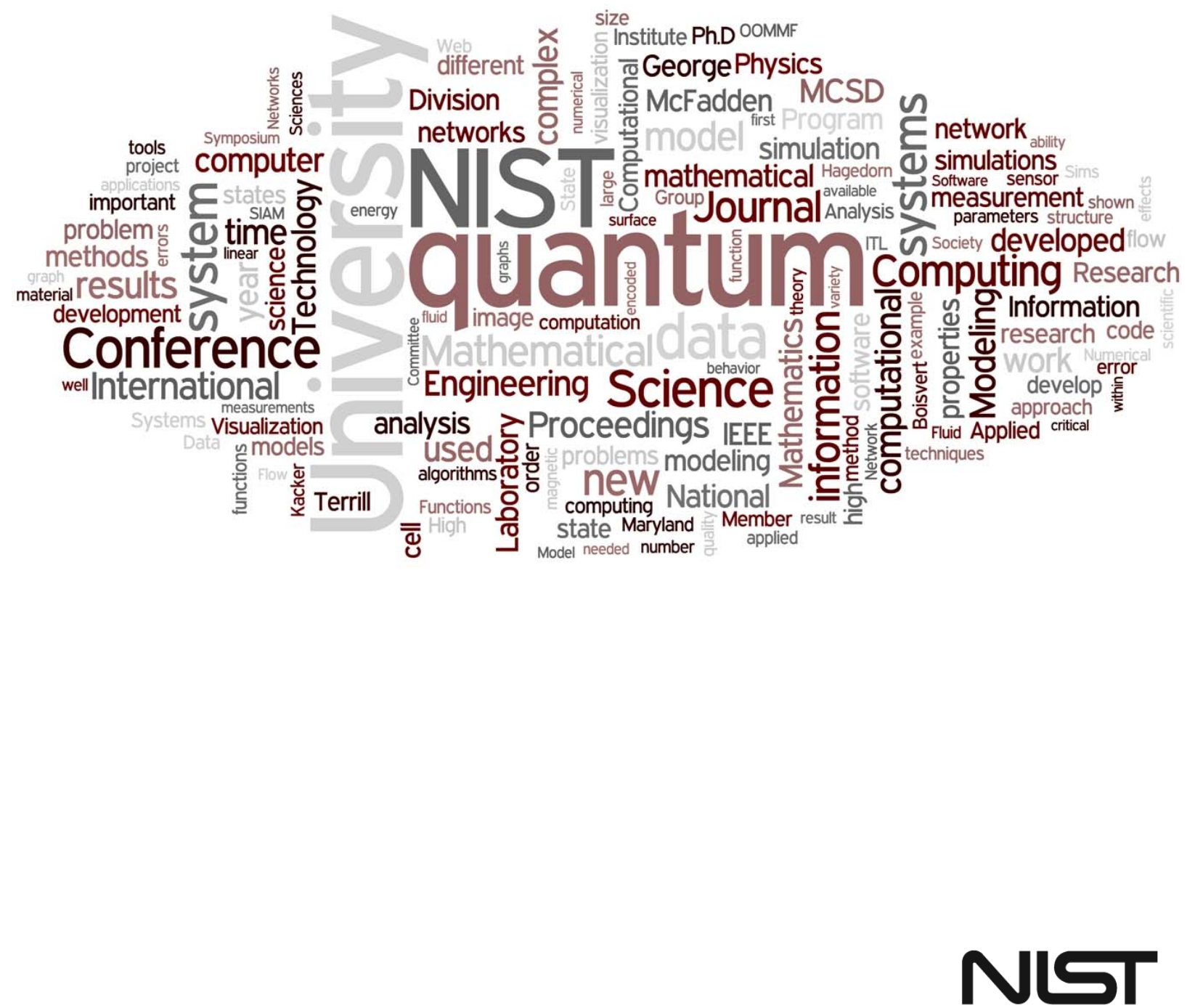

National Institute of Standards and Technology U.S. Department of Commerce 



\title{
Mathematical and Computational Sciences Division
}

\author{
Summary of Activities for Fiscal Year 2009
}

Ronald F. Boisvert, Editor Mathematical and Computational Sciences Division

Information Technology Laboratory

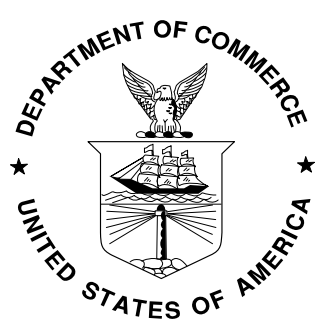

U.S. Department of Commerce Gary Locke, Secretary 


\section{$\underline{\text { Abstract }}$}

This report summarizes the technical work of the Mathematical and Computational Sciences Division (MCSD) of NIST's Information Technology Laboratory. Part I (Overview) provides a high-level overview of the Division's activities, including highlights of technical accomplishments during the previous year. Part II (Features) provides further details on ten projects of particular note this year. This is followed in Part III (Project Summaries) by brief synopses of all technical projects active during the past year. Part IV (Activity Data) provides listings of publications, technical talks, and other professional activities in which Division staff members have participated. The reporting period covered by this document is October 2008 through December 2009.

For further information, contact Ronald F. Boisvert, Mail Stop 8910, NIST, Gaithersburg, MD 208998910, phone 301-975-3812, email boisvert@nist.gov, or see the Division's web site at http://wWw.nist.gov/itl/math/index.cfm.

Cover Visualization: A “word cloud” summary of this report. See http://www.wordle.net/.

Acknowledgement: We are grateful to Robin Bickel for collecting the information and organizing the first draft of this report.

Disclaimer: Certain commercial entities, equipment, or materials may be identified in this document in order to describe an experimental procedure or concept adequately. Such identification is not intended to imply recommendation or endorsement by the National Institute of Standards and Technology, nor is it intended to imply that the entities, materials, or equipment are necessarily the best available for the purpose. 


\section{Contents}

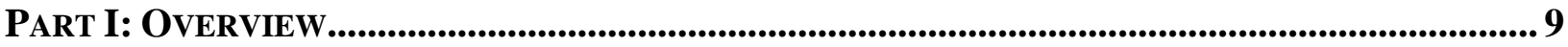

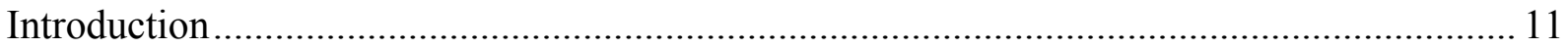

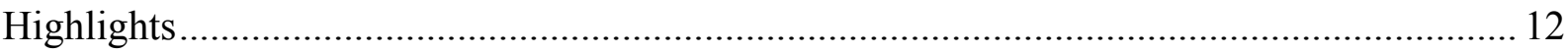

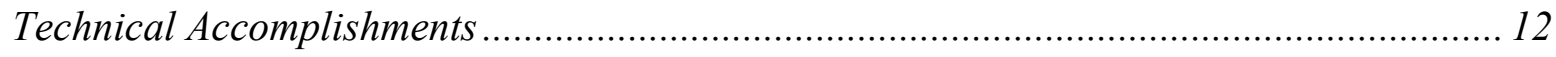

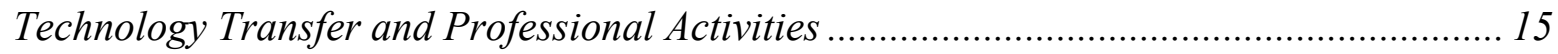

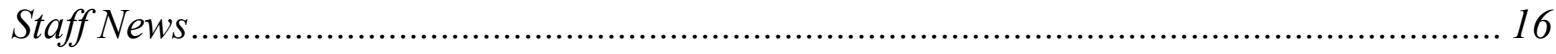

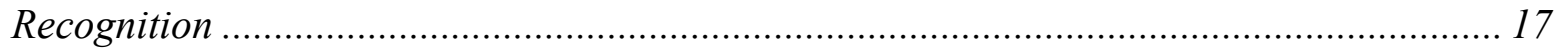

Restrictions on Transversal Encoded Quantum Gate Sets........................................................ 23

High Precision Calculations of Fundamental Properties of Few-electron Systems................... 25

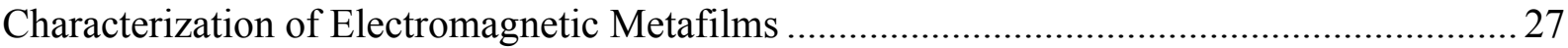

Recovering from Physically Impossible Reconstructions in Blind Deconvolution................... 29

A Quality Pre-Processor for Biological Cell Images ............................................................. 31

Computational Modeling of the Flow of Dense Suspensions.................................................... 33

Visualization of Radio Frequency Propagation for Body Area Networks................................. 35

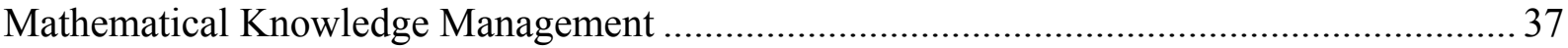

PART III: PROJECT SUMMARIES.......................................................................................................... 39

Information Technology Laboratory Programs .................................................................. 41

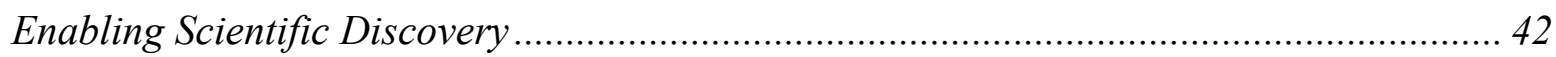

Recovering from Physically Impossible Reconstructions in Variational Blind

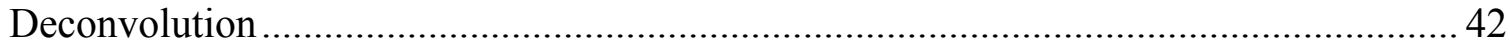

Computational Modeling of the Flow of Dense Suspensions ......................................... 42

Sparse Representations in High Dimensional Geometry ................................................. 42

Analysis of Diffusion and Stress in Binary Alloys ......................................................... 43

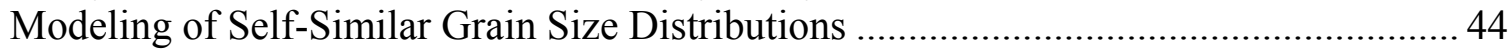

Modeling Fluid Flow in Materials Processing ................................................................... 44

Materials Data and Metrology for Applications to Machining Processes, Frangible

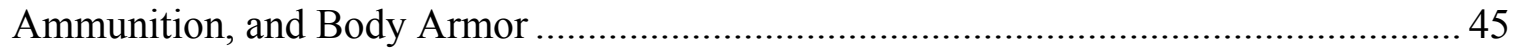

Boundary Slip Effects on the Stability of Spiral Poiseuille Flow ..................................... 46

Nanostructures, Nanooptics, and How to Squeeze the Light out of Quantum Dots......... 46

Computational Modeling and Visualization of Cement Paste Hydration and

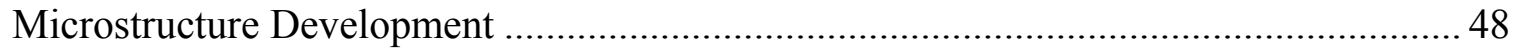

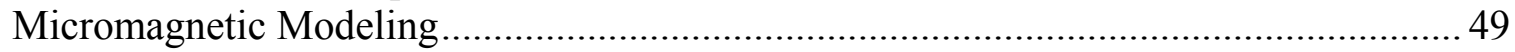

OOF: Finite Element Analysis of Material Microstructures............................................5 50

Fast Solvers for Models of Fluid Flow …………………............................................ 51

Parallel Adaptive Refinement and Multigrid Finite Element Methods .............................51 


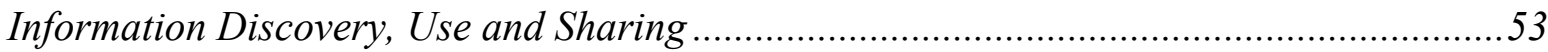

Mathematical Knowledge Management ......................................................................53

A Quality Pre-Processor for Biological Cell Images....................................................53

Estimating Volumes of Simulated Lung Cancer Nodules .................................................53

Standardization of Lung Cancer Growth Measurements................................................54

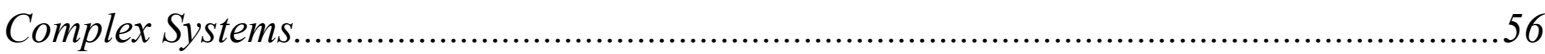

Methods for Characterizing Complex Networks .............................................................56

Standard Reference Data for Complex Network Research ………...................................56

Community Detection in Network Graphs ..............................................................58

Visualization of Large Complex Networks .................................................................58

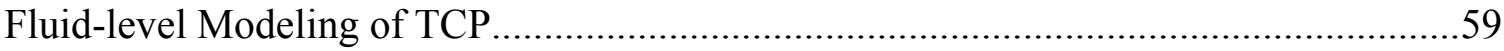

Analysis of a Distributed Protocol for Network Control..................................................59

Visualization of Possible Route Probability Distributions ...................................................60

Grid Network Simulation Using Piece-Wise Homogeneous Markov Chains...................61

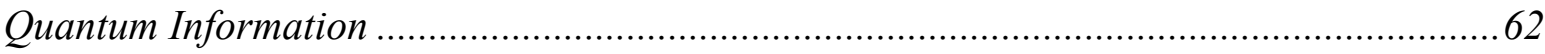

Restrictions on Transversal Encoded Quantum Gate Sets ...............................................62

Quantum Computing Theory ………………………..............................................62

Fault-tolerant Quantum Computation ........................................................................63

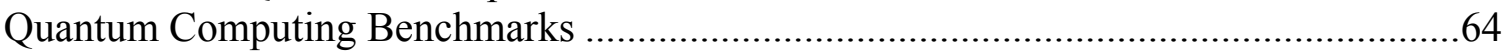

Quantum State Estimation Theory and Applications …………........................................64

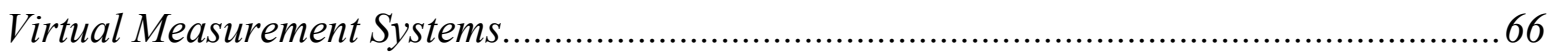

High Precision Calculations of Fundamental Properties of Few-electron Systems ..........66

Micromagnetic Modeling ……………………………............................................66

Uncertainty in Virtual Measurements from Quantum Chemistry Models .......................67

Pervasive Information Technology ..............................................................................6

Visualization of Radio Frequency Propagation for Body Area Networks ........................68

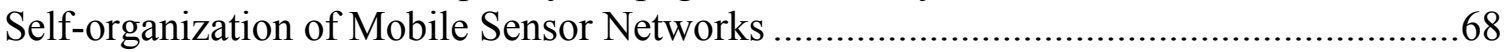

Trustworthy Information Systems ..........................................................................

Automated Combinatorial Testing for Software-systems (ACTS) …………………........70

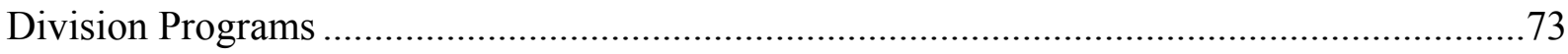

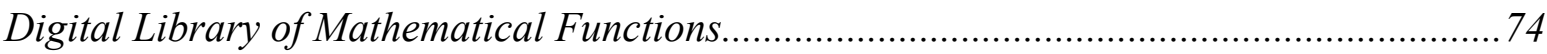

Digital Library of Mathematical Functions ......................................................................74

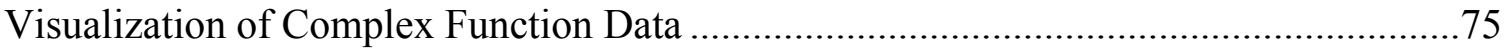

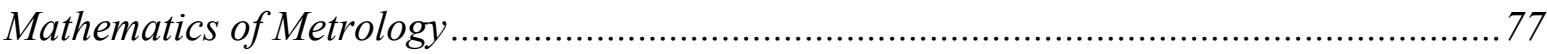

Multihazard Structural Optimization ................................................................................77

A Mathematical Model of Atmospheric Retention of Man-made $\mathrm{CO}_{2}$ Emissions ...........77

Simulation of Bioregulatory Networks Involved in Cell Cycle Control ...........................79

Modeling the Fluid Dynamics of Tear Films on the Cornea .............................................79

Modeling Equilibrium, Stability and Transport during Magnetic Fusion ...........................80

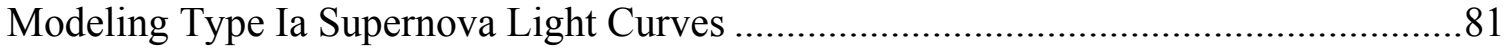

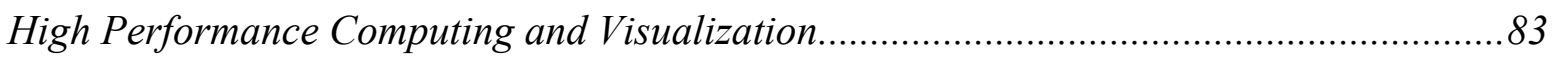

Visualizing Fire and the Resulting Deformation of Structures .........................................83

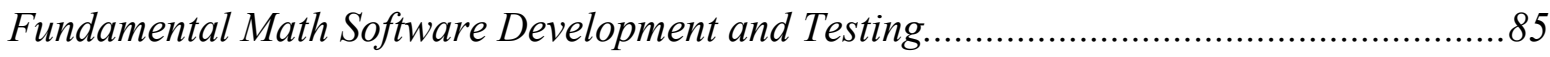

SciMark, a Web-based Benchmark for Numerical Computing in Java..............................85

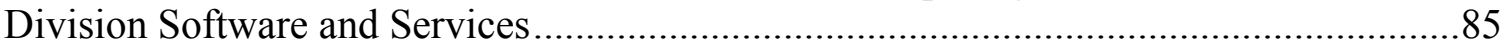




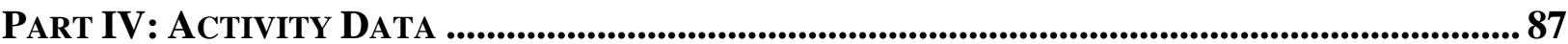

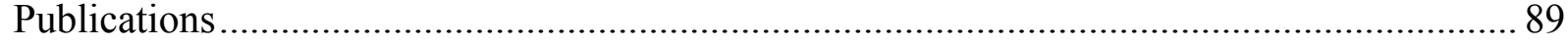

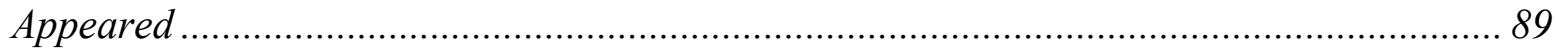

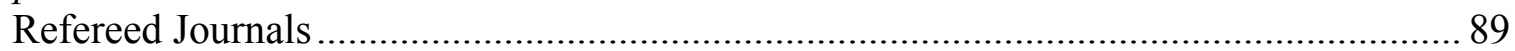

Journal of Research of NIST ................................................................................ 90

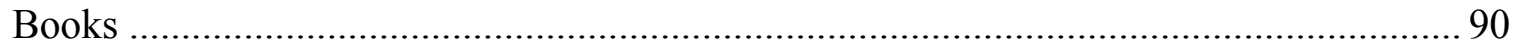

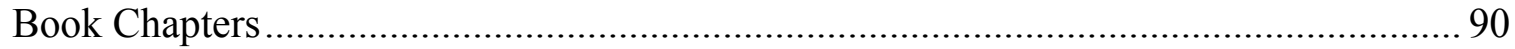

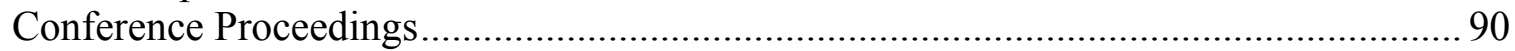

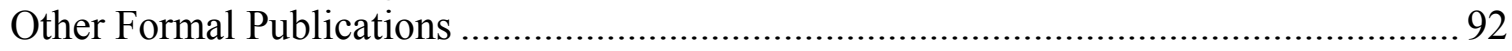

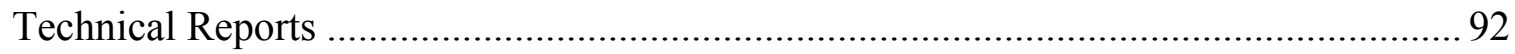

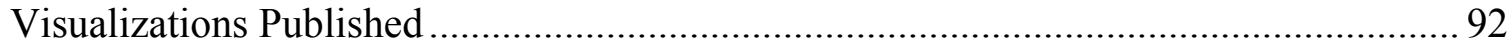

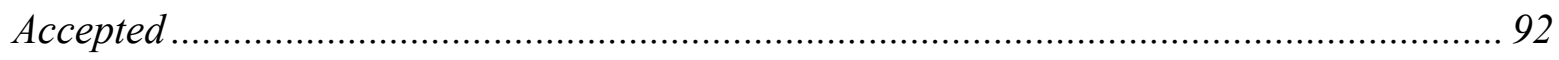

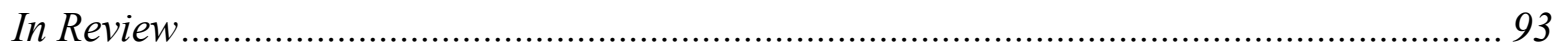

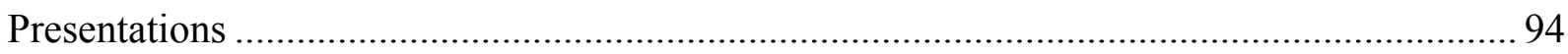

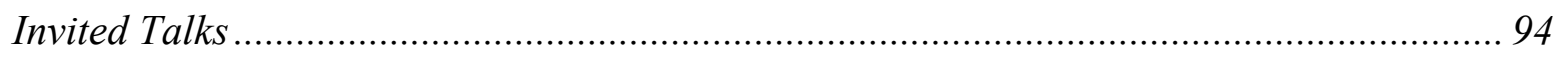

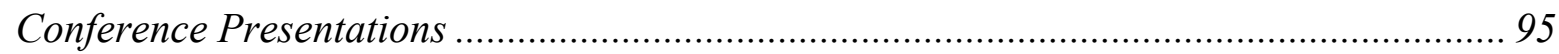

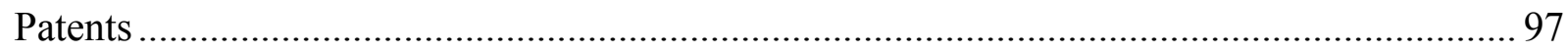

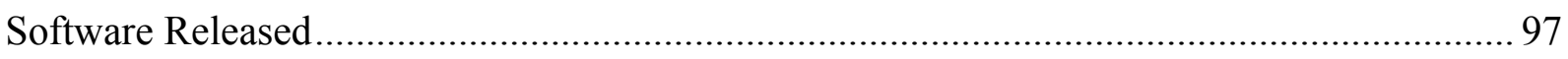

Conferences, Minisymposia, Lecture Series, Shortcourses ................................................ 98

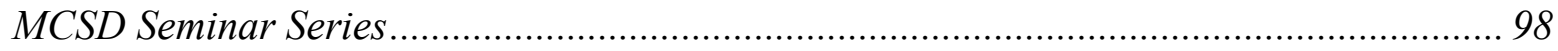

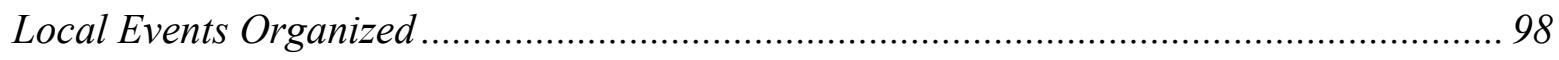

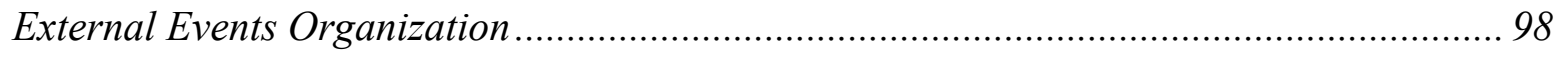

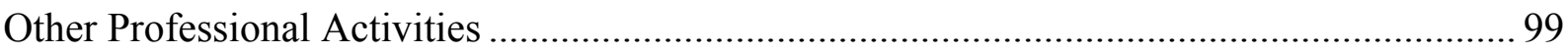

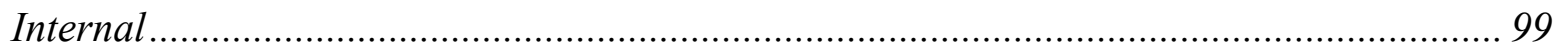

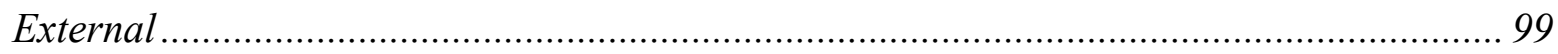

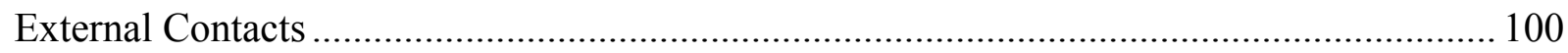

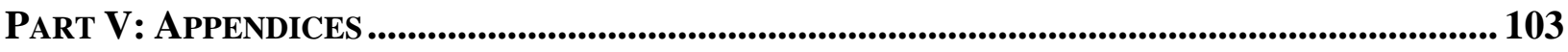

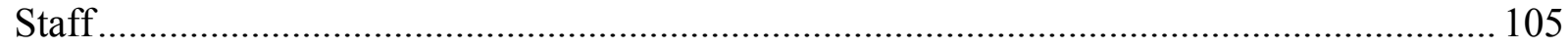

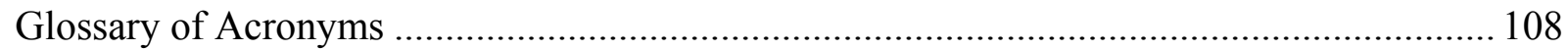




\section{Part I}

\section{Overview}

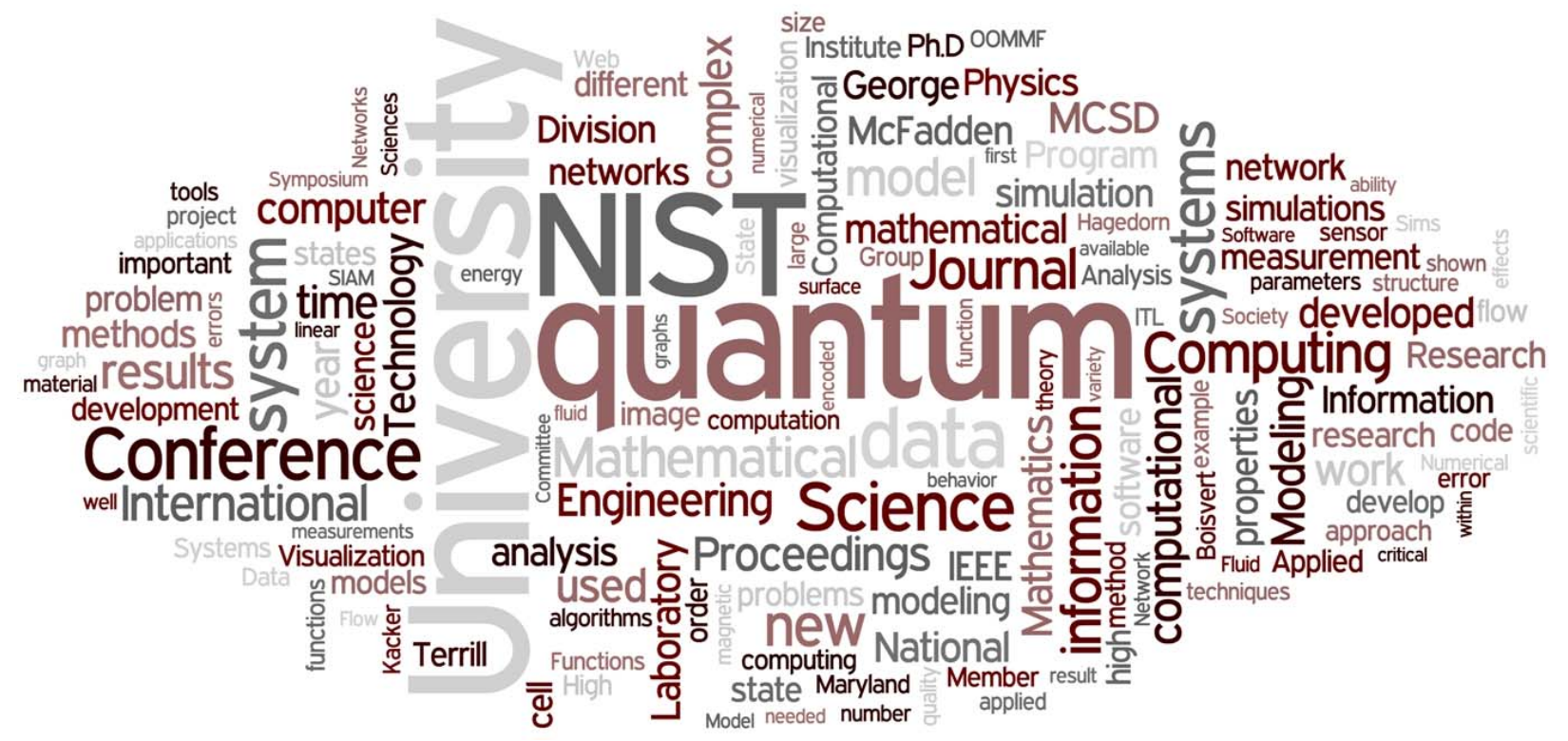




\section{Introduction}

Founded in 1901, NIST is a non-regulatory federal agency within the U.S. Department of Commerce. NIST's mission is to promote U.S. innovation and industrial competitiveness by advancing measurement science, standards, and technology in ways that enhance economic security and improve our quality of life. The NIST Laboratory program is broad-ranging, with research efforts in physics, electronics and electrical engineering, chemical science and technology, materials science, building and fire research, manufacturing engineering, and information technology.

The Mathematical and Computational Sciences Division (MCSD) provides leadership within NIST in the application of applied and computational mathematics to the solution to science and engineering problems arising in measurement science. In that role Division staff members

- perform research and development in applied mathematics and computer science and engineering, including analytical methods, numerical algorithms, advanced computing and communications architectures and applications, and high performance visualization;

- engage in peer-to-peer collaborations in the application of mathematical and computational technologies to NIST problems;

- develop and disseminate mathematical reference data, software, and related tools; and

- work with internal and external groups to develop standards, test procedures, reference implementations, and other measurement technologies for advanced scientific computation on current and future architectures.

Division staff is organized into four groups:

\section{Mathematical Modeling Group (Geoffrey McFadden, Leader)}

Performs research and maintains expertise in applied mathematics, mathematical modeling, numerical analysis, and scientific computing for application to multidisciplinary problems of interest to the NIST measurement science program.

\section{Mathematical Software Group (Daniel Lozier, Leader)}

Performs research and maintains expertise in the methodology and application of mathematical algorithms and software in support of computational science within NIST as well as in industry and academia.

\section{Discrete Mathematical Analysis Group (Ronald Boisvert, Acting Leader)}

Performs research and maintains expertise in the theory, methodology, and application of discrete mathematics for application to the NIST measurement science program, including complex systems and quantum information science.

\section{Scientific Applications and Visualization Group (Judith Terrill, Leader)}

Develops novel algorithms and implementations for parallel and distributed computation and visual data analysis in a variety of hardware environments.

MCSD is one of six technical Divisions within the NIST Information Technology Laboratory (ITL). The work of ITL is organized into 11 cross-cutting programmatic areas, seven of which have significant MCSD participation. See "ITL Programs in Brief" sidebar. MCSD Division-centric work is organized into four additional themes. See the "MCSD Programs in Brief" sidebar. Division programs are characterized as mature Division-centric efforts in support of NIST goals that do not fit in any of ITL's Program areas, but are nevertheless critical for NIST metrology. Other Division efforts fulfill a need to continuously develop and maintain technical competence and facilities in core areas of mathematics and computational science which are broadly applicable.

In Part III of this document we provide descriptions of most ongoing technical projects of the Division. These are organized under the Program areas listed in the sidebars. 


\section{ITL Programs In Brief}

* Complex Systems. Complex Systems are composed of large interrelated, interacting entities which taken together, exhibit a macroscopic behavior which is not predictable by examining the components. This Program seeks to understand the fundamental science of these systems and develop rigorous descriptions that enable prediction and control of their behavior.

Cyber and Network Security. Cybersecurity is focused on ensuring three IT system objectives: confidentiality, integrity, and availability. This Program addresses long-term scientific issues in some of the building blocks of IT security - cryptography, security testing and evaluation, security metrics, and security properties - providing a more scientific foundation for computer and network security, while maintaining a focus on near-term issues.

* Enabling Scientific Discovery. Modern scientific research has become more and more dependent on mathematical, statistical, and computational tools. This Program promotes the use of these tools to dramatically advance our ability to predict the behavior of a broad range of complex scientific and engineering systems and processes. In particular, this Program focuses on inter-disciplinary projects that involve novel computational statistics and simulation methods and software.

Identity Management Systems. Identity management systems are responsible for the creation, use, and termination of electronic identities used to access logical and physical resources. This Program is pursuing the development of common models and metrics for identity management, critical standards, and interoperability of electronic identities. These efforts will improve the quality, usability, and consistency of identity management systems while protecting privacy.

* Information Discovery, Use, and Sharing. This Program fosters innovation throughout the information life cycle by developing the measurement infrastructure to enhance knowledge discovery, information exchange, and information usability. The Program enables novel computational approaches to data collection and analysis to be combined with improved interoperability techniques to effectively extract needed information from the wealth of available data.

* Pervasive Information Technologies. Pervasive information technology is the trend towards increasingly ubiquitous connected computing sensors, devices, and networks that monitor and respond transparently to human needs. This Program facilitates the creation of standards for sensor communication, networking interoperability, and sensor information security.
* Trustworthy Information Systems. Trustworthy information systems perform as intended for a specific purpose, with operational resiliency and without unwanted side effects or exploitable vulnerabilities. This Program will improve the ability to model, produce, measure, and assess trustworthiness through innovative technologies, models, measurements, and software tools.

* Virtual Measurement Systems. A virtual measurement is a quantitative result and its uncertainty, obtained by a computer simulation or computer-assisted measurements. This Program introduces metrology constructs, standard references, uncertainty characterization, and traceability into scientific computation and computer-assisted measurements to enable predictive computing with quantified reliability.

* Ouantum Information. An emerging new discipline at the intersection of quantum mechanics and computer science, quantum information science (QIS) is likely to revolutionize science and technology in the same way that lasers, electronics, and computing did in the $20^{\text {th }}$ century, including phenomenal increases in information storage and processing speed and communication channels with extremely high levels of security. However, the needed manipulation and control of quantum states remains a grand challenge. We are developing theoretical underpinning to support the multi-laboratory experimental program in QIS at NIST.

Health Information Technology. The demand for online medical information and simplified, standardized methods to access healthcare information and services is crucial in making healthcare safe and available to all. NIST is collaborating with industry, standards organizations, consortia, and government agencies to build tools and prototypes to advance the adoption of IT within healthcare systems.

Voting. The 2002 Help America Vote Act gives NIST a key role in realizing nationwide improvements in voting systems. To assist the Election Assistance Commission with the development of voluntary voting system guidelines, HAVA established the Technical Guidelines Development Committee (TGDC) and directs NIST to chair the TGDC. NIST research activities include: security of computers, computer networks, and computer data storage used in voting systems; methods to detect and prevent fraud; protection of voter privacy; and the role of human factors and assistive technologies in the design and application of voting systems.

* indicates MCSD participation

\section{Highlights}

In this section we identify some of the major accomplishments of the Division during the past year. We also provide news related to MCSD staff.

\section{Technical Accomplishments}

MCSD has made significant technical progress on many fronts during the past year. Here we highlight a few examples. Further details are provided in Part II (Features) and Part III (Project Summaries) of this document. 


\section{MCSD Programs In Brief}

Digital Library of Mathematical Functions. The special functions of applied mathematics are fundamental tools enabling modeling and analysis in all areas of science and engineering. To make effective use of such functions, practitioners must have ready access to a reliable source of information on their properties. The goal of this work is the development and dissemination of definitive reference data on the special functions of applied mathematics. The DLMF will be a freely available interactive and richly linked online resource.

Mathematics of Metrology. Mathematics plays an important role in the science of metrology. Mathematical models are needed to understand how to design effective measurement systems, and to analyze the results they produce. Mathematical techniques are used to develop and analyze idealized models of physical phenomena to be measured, and mathematical algorithms are necessary to find optimal system parameters. Finally, mathematical and statistical techniques are needed to transform the resulting data into useful information. The goal of this work is to develop fundamental mathematical methods and analytical tools necessary for NIST to continue as a world-class metrology institute, and to apply them critical measurement science applications.
Fundamental Math Software Development, Testing. Modern science and engineering in general, and modern measurement science in particular require a wide variety of software tools for scientific discovery, exploration, and analysis. As scientific inquiry becomes deeper and more specialized, so must the supporting software tools. The goal of this work is to develop critical software tools that support measurement science at NIST, as well as computational science and engineering at large.

High Performance Computing and Visualization. Computational capability is advancing rapidly. This means that modeling and simulation can be done with greatly increased fidelity (e.g. higher resolution, more complex physics). However, developing largescale parallel applications remains highly challenging, requiring expertise that application scientists rarely have.In addition, the hardware landscape is changing rapidly so new algorithmic techniques must constantly be developed. We are developing and applying facilities and expertise of this type for application to NIST problems. Large scale computations and laboratory experiments invariably produce large volumes of scientific data, which cannot be readily comprehended without some form of visual analysis. We are developing the infrastructure necessary for advanced visualization of scientific data, including the use of 3D immersive environments and applying this to NIST problems. One of our goals is to develop the 3D immersive environment into a true interactive measurement laboratory.

Image analysis continues to be a strong emphasis of Division activity. Imagery requiring enhancement and analysis in NIST applications arise in nanotechnology, medicine, biology, and materials science. For example, we are working with researchers in the NIST Chemical Sciences and Technology Laboratory to develop effective automated analysis of cell imagery. In collaboration with Karen Kafadar of Indiana University and Alden Dima of ITL, Adele Peskin has developed a quality metric for cell imagery. The metric is a useful predictor of which of the many available segmentation algorithms will be most effective in isolating an individual cell within an image. [Page 31] Images also play an important role in the analysis of complex materials. MCSD's OOF system starts with a micrograph of a complex material from which it develops a computational grid to enable a finite element simulation from which macroscopic properties can be derived. This year substantial progress has been made in extending OOF's capabilities to three-dimensional image-based analysis. [Page 50] We also take the lead in the development of new image analysis methods for use in metrology. This year Alfred Carasso completed an insightful mathematical analysis of a recently proposed blind deconvolution algorithm, demonstrating that the visually compelling reconstructions that it produced were actually inadmissible, corresponding to physically impossible blurs. He identified the source of the problem and developed a correction procedure. The result has proven to be highly effective. [Page 29]

Division research in quantum information continues to contribute significantly to the NIST program in this area. Two such projects are externally funded efforts: Quantum Optical Metrology with N-Photons (joint with EEEL and PL) and Analog Quantum Integrated Circuits (joint with PL). One important contribution we are making to these efforts is the development of novel quantum state estimation procedures and associated software. [Page 64] Our efforts to improve our understanding of the potential for the development of quantum computing devices also continue. This year we settled a long-standing conjecture regarding the design of quantum gates sets. Bryan Eastin and Manny Knill showed that transversal encoded gate sets, which are ideal for error management, cannot be universal, and hence cannot solely be relied upon for the practical implementation of quantum computers. [Page 21]

We continue our efforts, begun in 2007, to develop foundations of measurement science for information systems. A significant focus of this work is the development and analysis of metrics for 
characterizing complex networks. One such example is the reliability polynomial, which gives the probability that a network will remain connected given that each edge can fail independently with a probability $p$. The reliability polynomial of a connected graph $G$ with $m$ edges is an $m^{\text {th }}$ degree polynomial in $p$ whose $j^{\text {th }}$ coefficient is the number of distinct connected subgraphs of $G$ with exactly $j$ edges. Unfortunately, determining these coefficients is computationally intractable. (In particular, the problem is \#Pcomplete.) Thus, the best we can expect to do in general is to approximate the polynomial. Brian Cloteaux and Isabel Beichl have developed a new method to do this. Their approach has two advantages. First, while there are several algorithms that provide pointwise approximations of the polynomial, their approach approximates the polynomial coefficients themselves. Secondly, their method has shown much faster rate of convergence than previously proposed methods. A paper has been accepted for publication. [Page 56]

This year also witnessed a significant milestone in our efforts to develop virtual measurement systems for fundamental atomic properties. Our recent computations of the nonrelativistic energies for excited states of the lithium atom represent the highest level of accuracy ever reached (less than $10^{-9}$ hartree) in atomic quantum computations with more than two electrons. The achievement was reported in an article in Physical Review A by James Sims of MCSD and Stanley Hagstrom of Indiana University. The judicious use of both parallel computing and multiple precision arithmetic (30+ digits) was critical to obtain such high accuracy results. In addition, Sims and Hagstrom developed an improved parallel computer code for a key computational bottleneck, high-precision solution of the large-scale generalized matrix eigenvalue problem. For a 17,190 term wave function they achieved a factor of 30 speedup on 32 processors of a local Linux cluster. The resulting computations obtained the values of four excited states of the lithium atom to an accuracy two orders of magnitude greater than has been done before. While the lithium calculation is valuable in itself, the deeper import of refining their method is that it should enable the calculation of excitation energies for beryllium, which has four electrons. This achievement would enable theorists to predict with greater accuracy values for all of the remaining elements in the second row of the periodic table. [Page 25]

Our efforts with the NIST Building and Fire Research Laboratory to develop a virtual cement and concrete testing laboratory reached a new level of maturity this year. Our simulations have enabled the exploration of the effects of particle size distribution, particle shape distribution, volume fraction of aggregates, and other relevant system parameters, on critical properties such as the yield stress and viscosity of suspensions. In order to perform simulations on systems of sufficient size, density, and simulated time, we have developed highly parallel implementation of our models. This implementation, which is based upon dissipative particle dynamics, has run simulations efficiently using more than 2,000 processors. In these cases we often simulate systems consisting of over 1 million mesoscopic fluid particles and up to 10,000 aggregates. These computations were enabled by DOE INCITE awards of 750,000 hours of CPU time on the IBM Blue Gene/P machine at the Argonne National Laboratory Leadership Computing Facility in each of 2008 and 2009. For 2010 we have been awarded an additional 2 million CPU-hours of compute time. In 2009 William George and Judith Terrill of MCSD were part of a team awarded the DOC Silver Medal in recognition of this work. [Page 17]

Visual analysis plays an important role in our modeling of suspensions. Such analysis is supported by our immersive visualization facilities in both Gaithersburg and Boulder. Division staff members work closely with scientists from a variety of NIST Labs to develop novel visualizations that enable insights that would be difficult to obtain otherwise. Examples of projects that have benefited from this facility include ITL efforts to model radio frequency propagation in body area networks [Page 35], work with PL to model the optical properties of nanostructures [Page 46], ITL efforts to assess community detection algorithms for large-scale graphs [Page 56], and work with BFRL on modeling the deformation of structures resulting from fire [Page 83]. Our visualization team is continuously updating our hardware and software capabilities. In particular, we have developed an extensive suite of visualization primitives and tools that allow us to be both fast and flexible in our work. These tools run without change on a range of platforms from a laptop to our immersive environment. A recent focus has been the use of specialized graphics processing unit (GPU) processing to provide novel capabilities for our most challenging visualization problems. 
Table 1. Downloads of Selected Division Software Packages During CY 2009

\begin{tabular}{llr}
\hline \multicolumn{1}{c}{ Package } & \multicolumn{1}{c}{ Description } & Downloads \\
\hline JAMA & Linear algebra in Java & 17,067 \\
\hline TNT & Linear algebra using C++ templates & 15,312 \\
\hline LAPACK++ & Linear algebra in C++ & 6,307 \\
\hline OOMMF & Modeling of nano-magnetic phenomena & 5,200 \\
\hline SparseLib++ & Sparse linear algebra in C++ & 4,903 \\
\hline f90gl & Fortran 90 interface to OpenGL graphics & 3,530 \\
\hline REGEN & Modeling of cryocoolers & 1,932 \\
\hline spblas & Sparse basic linear algebra components & 1,569 \\
\hline LaTeXML & LaTeX to MathML translator & 1,520 \\
\hline OOF & Modeling materials with complex microstructure & 1,204 \\
\hline PHAML & Parallel adaptive solution of partial differential equations & 767 \\
\hline
\end{tabular}

Finally, we are happy to report that the release of the long awaited Digital Library of Mathematical Functions (DLMF) is in sight. A modern successor to the classic Handbook of Mathematical Functions (M. Abramowitz and I. Stegun, Eds., 1964), the DLMF is a carefully constructed reference on the properties of the special functions of applied mathematics which has been compiled with the cooperation of some 50 researchers worldwide. Release is now scheduled for the spring of 2010 . The information will be made available as both an innovative interactive web resource as well as a traditional printed book produced in cooperation with Cambridge University Press. [Page 74]

\section{Technology Transfer and Professional Activities}

The volume of technical output of MCSD remains high. During the last 18 months, Division staff members were (co-)authors of 24 articles appearing in peer-reviewed journals, two books, two book chapters, 26 papers in conference proceedings, and 7 other formal publications. Twenty-five additional papers have been accepted for publication, while 19 others are undergoing review. Division staff members gave 27 invited technical talks and presented 40 others in conferences and workshops.

MCSD continues to maintain an active Web site with a variety of information and services, including the Guide to Available Mathematical Software, the Matrix Market, and the SciMark Java benchmark. During calendar year 2009, the division web server satisfied more than 7 million requests for pages from more than 445,000 distinct hosts. On a daily basis, more than 19,000 pages and 1.6 Gbytes of data were shipped. In total, there have been more than 217 million "hits" on MCSD Web servers since they went online as NIST's first web servers in 1994. The individual software packages that we produce and distribute also continue to see very high usage. Table 1 lists download counts for some of our most popular ones. Another indication of the successful transfer of our technology is references to our software in refereed journal articles. For example, our OOMMF software for nano-magnetic modeling was cited in 120 such papers which were published in FY 2009 alone (836 such papers have been identified since 2001).

Members of the Division are also active in professional circles. Isabel Beichl began a term as Editor-in-Chief of Computing in Science \& Engineering in January 2009. Staff members hold a total of six additional associate editorships of peer-reviewed journals. They are also active in conference organization, serving on five organizing/steering/program committees. Staff members organized five minisymposia at technical conferences.

Service within professional societies is also prevalent among our staff. For example, Ronald Boisvert serves as Co-Chair of the Publications Board of the Association for Computing Machinery (ACM) and is a member of the ACM Council, the association's board of directors. Daniel Lozier serves as Secretary of the Society for Industrial and Applied Mathematics (SIAM) Activity Group on Orthogonal Polynomials and Special Functions. Staff members are also active in a variety of working groups. For example, Ronald Boisvert serves as Chair of the International Federation for Information Processing (IFIP) Working Group 2.5 on Numerical Software, Donald Porter is a member of the Tcl Core Team, and Bruce Miller is a member of W3C's Math Working Group. Judith Terrill represents NIST on the High 
Table 2. Student Interns in MCSD

\begin{tabular}{|c|c|c|c|c|c|}
\hline Name & Institution & Level & Program & Mentor & Project Title \\
\hline $\begin{array}{l}\text { Matthew } \\
\text { Du }\end{array}$ & $\begin{array}{l}\text { Thomas S. } \\
\text { Wooton } \\
\text { High School }\end{array}$ & $\begin{array}{l}\text { High } \\
\text { School }\end{array}$ & $\begin{array}{l}\text { Student } \\
\text { Volunteer }\end{array}$ & J. Terrill & $\begin{array}{l}\text { Modeling Window Breakage due to } \\
\text { Fire using the Open Dynamics En- } \\
\text { gine }\end{array}$ \\
\hline $\begin{array}{l}\text { Julie } \\
\text { Eisenberg }\end{array}$ & $\begin{array}{l}\text { Damascus } \\
\text { High School }\end{array}$ & $\begin{array}{l}\text { High } \\
\text { School }\end{array}$ & $\begin{array}{l}\text { Student } \\
\text { Volunteer }\end{array}$ & J. Terrill & $\begin{array}{l}\text { Merging Data Mining with Visuali- } \\
\text { zation }\end{array}$ \\
\hline $\begin{array}{l}\text { Daniel } \\
\text { Leifer }\end{array}$ & $\begin{array}{l}\text { Stanford } \\
\text { Univ. }\end{array}$ & $\begin{array}{l}\text { Under- } \\
\text { graduate }\end{array}$ & SURF & A. Peskin & $\begin{array}{l}\text { Development of Standardized Lung } \\
\text { Tumor Data Sets }\end{array}$ \\
\hline $\begin{array}{l}\text { Sei } \\
\text { Masouka }\end{array}$ & $\begin{array}{l}\text { Winston } \\
\text { Churchill } \\
\text { High School }\end{array}$ & $\begin{array}{l}\text { High } \\
\text { School }\end{array}$ & $\begin{array}{l}\text { Student } \\
\text { Volunteer }\end{array}$ & J. Terrill & $\begin{array}{l}\text { Influence of Particle Size Distribu- } \\
\text { tion on Setting of Tricalcium Silicate }\end{array}$ \\
\hline $\begin{array}{l}\text { Russell } \\
\text { McFadden }\end{array}$ & $\begin{array}{l}\text { St. Olaf Col- } \\
\text { lege }\end{array}$ & $\begin{array}{l}\text { Under- } \\
\text { graduate }\end{array}$ & $\begin{array}{l}\text { Student } \\
\text { Volunteer }\end{array}$ & W. Mitchell & $\begin{array}{l}\text { Mathematical Modeling and Soft- } \\
\text { ware }\end{array}$ \\
\hline $\begin{array}{l}\text { Adam } \\
\text { Meier }\end{array}$ & $\begin{array}{l}\text { University of } \\
\text { Colorado }\end{array}$ & Graduate & PREP & E. Knill & Quantum Computer Simulation \\
\hline $\begin{array}{l}\text { Menal } \\
\text { Modha }\end{array}$ & UMBC & $\begin{array}{l}\text { Under- } \\
\text { graduate }\end{array}$ & SURF & $\begin{array}{l}\text { R. Kacker/ } \\
\text { R. Kuhn }\end{array}$ & $\begin{array}{l}\text { Isolating Failure-causing Combina- } \\
\text { tions in Software Discovered via } \\
\text { Combinatorial Testing }\end{array}$ \\
\hline $\begin{array}{l}\text { Katherine } \\
\text { Morrison } \\
\end{array}$ & $\begin{array}{l}\text { Univ. of } \\
\text { Nebraska } \\
\end{array}$ & Graduate & $\begin{array}{l}\text { Guest Re- } \\
\text { searcher }\end{array}$ & F. Hunt & $\begin{array}{l}\text { Modeling the Operation of a Grid } \\
\text { Computing System }\end{array}$ \\
\hline $\begin{array}{l}\text { Michael } \\
\text { Mullan }\end{array}$ & $\begin{array}{l}\text { University of } \\
\text { Colorado }\end{array}$ & Graduate & PREP & E. Knill & Quantum Computing Theory \\
\hline $\begin{array}{l}\text { Sanjeev } \\
\text { Muralidharan }\end{array}$ & $\begin{array}{l}\text { Montgomery } \\
\text { Blair High } \\
\text { School }\end{array}$ & $\begin{array}{l}\text { High } \\
\text { School }\end{array}$ & $\begin{array}{l}\text { Student } \\
\text { Volunteer }\end{array}$ & J. Terrill & $\begin{array}{l}\text { Visualization and Measurement in } \\
\text { Dental Images }\end{array}$ \\
\hline $\begin{array}{l}\text { Michael } \\
\text { Sharpnack }\end{array}$ & NYU & $\begin{array}{l}\text { Under- } \\
\text { graduate }\end{array}$ & SURF & $\begin{array}{l}\text { G. McFad- } \\
\text { den }\end{array}$ & $\begin{array}{l}\text { Sensitivity Analysis for a Biological } \\
\text { Model of Cellular Response to DNS } \\
\text { Damage }\end{array}$ \\
\hline $\begin{array}{l}\text { Nadezhda } \\
\text { Serova }\end{array}$ & UMBC & $\begin{array}{l}\text { Under- } \\
\text { graduate }\end{array}$ & SURF & B. Cloteaux & $\begin{array}{l}\text { Approximation Schemes for the } \\
\text { Maximal Cover Sum Problem }\end{array}$ \\
\hline $\begin{array}{l}\text { Joseph } \\
\text { Owen }\end{array}$ & $\begin{array}{l}\text { Montgomery } \\
\text { Blair High } \\
\text { School }\end{array}$ & $\begin{array}{l}\text { High } \\
\text { School }\end{array}$ & $\begin{array}{l}\text { Student } \\
\text { Volunteer }\end{array}$ & J. Terrill & $\begin{array}{l}\text { Language for Generating Geometry } \\
\text { for the Case of Room and Contents } \\
\text { in the Project "Impact of Fire on } \\
\text { Building Structures" }\end{array}$ \\
\hline $\begin{array}{l}\text { Jennifer } \\
\text { Schiller }\end{array}$ & $\begin{array}{l}\text { Reservoir } \\
\text { High School }\end{array}$ & $\begin{array}{l}\text { High } \\
\text { School }\end{array}$ & $\begin{array}{l}\text { Student } \\
\text { Volunteer }\end{array}$ & J. Terrill & $\begin{array}{l}\text { A Mathematical Metric for Stress } \\
\text { Groupings in Flow of Suspensions }\end{array}$ \\
\hline $\begin{array}{l}\text { Yanbao } \\
\text { Zhang }\end{array}$ & $\begin{array}{l}\text { University of } \\
\text { Colorado }\end{array}$ & Graduate & PREP & E. Knill & Tests of Local Realism \\
\hline
\end{tabular}

SURF: NIST Student Undergraduate Student Fellowship Program.

PREP: NIST Professional Research Experience Program

End Computing Interagency Working Group of the Federal Networking and Information Technology Research and Development (NITRD) Program.

For further details, see Part IV (Activity Data) of this document.

\section{Staff News}

During FY 2009 MCSD welcomed a new National Research Council (NRC) Postdoctoral Associate, James Benson of the University of Missouri - Columbia. A recent PhD in Mathematics, Benson's thesis was in the mathematics of cryobiology, the study of biological systems at low temperatures. In particular, 
he developed methods for optimizing cryobiological protocols, and in the process defined a new model for common cryobiological procedures. At NIST Benson will be working with Anthony Kearsley. Benson's arrival brings our current number of NRC Postdoctoral Associates to four.

During FY 2009 MCSD was able to support the work of 15 student interns, including four in the Student Undergraduate Research Fellowship (SURF) program, three graduate students in Boulder's Professional Research Experience Program (PREP), one graduate student guest researcher, and seven high school volunteers. See Table 2 for a complete list.

\section{Recognition}

Division staff garnered a significant number of professional recognitions during the past year. These are described below.

\section{External Awards}

o American Physical Society Outstanding Referee. Manny Knill of MCSD has been cited as an Outstanding Referee by the American Physical Society (APS). This lifetime APS award was initiated in 2008 "to recognize scientists who have been exceptionally helpful in assessing manuscripts for publication in the APS journals." The program is designed to annually recognize about 130 of APS's approximately 42,000 referees, though larger numbers have been identified during

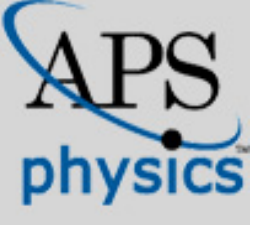
2008 and 2009, the program's first two "catch-up years." The basis for selection was the quality, number and timeliness of their reports, without regard for membership in the APS, country of origin, or field of research.

o SIAM Fellows. Dianne O'Leary and G. W. (Pete) Stewart, Faculty Appointees in MCSD, have been named Fellows of the Society for Industrial and Applied Mathematics (SIAM). O'Leary, who is Professor of Computer Science at the University of Maryland College Park

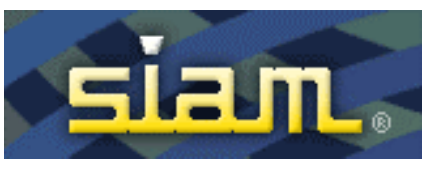
(UMCP), was cited for contributions to linear algebra, regularization, and applications. Stewart, Distinguished University Professor Emeritus of Computer Science at UMCP, was cited for contributions to numerical linear algebra. The SIAM Fellows program has just been established; O'Leary and Stewart are members of the inaugural class of inductees.

o Excellence in Technology Transfer. Raghu Kacker of MCSD, Rick Kuhn of the ITL Computer Security Division, Jeff Yu Lei of the University of Texas at Arlington (and a guest researcher in MCSD), and James Lawrence of George Mason University (and a faculty appointee in MCSD) were selected as recipients of the 2009 Federal Laboratory Consortium-Mid Atlantic Regional "Excellence in Technology Transfer" Award for their work in "Advanced Combinatorial Test Suites (ACTS) for Testing Software." This prestigious award recognizes employees who have accomplished outstanding work in the process of transferring a technology developed by a Federal laboratory to the commercial marketplace.

\section{Internal Awards}

o Silver Medal. William George and Judith Terrill of MCSD are co-winners of a 2009 Silver Medal from the Department of Commerce for their work on the modeling and simulation of complex suspensions. The Silver Medal recognizes "exceptional performance characterized by noteworthy or superlative contributions which have a direct and lasting impact within the Depart-

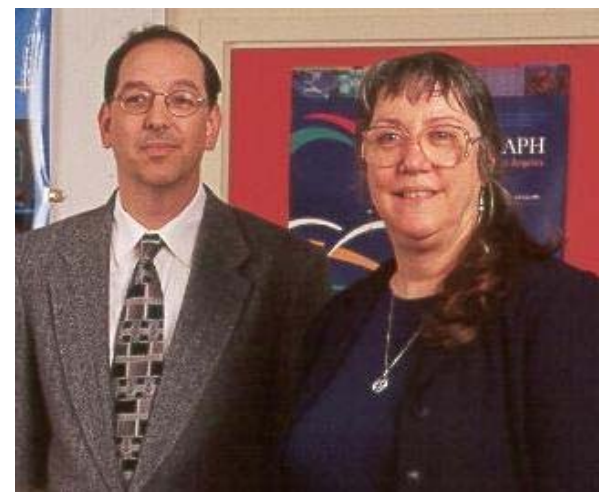

William George and Judith Terrill received the Department of Commerce's Silver Medal 
ment." Dale Bentz, Jeffrey Bullard, Edward Garboczi, and Nicos Martys of BFRL were co-winners. The award citation credits the group wih the creation of the virtual cement and concrete testing laboratory (VCCTL), "which uses intensive computer simulation to mimic a physical testing laboratory and has proven to be capable of revealing behavior beyond the limits of traditional testing instruments."

o Allen V. Astin Measurement Science Award. Andrew Dientsfrey of MCSD is a co-winner of the 2009 Allen V. Astin Measurement Science Award for work in waveform metrology. The award was instituted to recognize "outstanding achievement in the advancement of measurement science or in the delivery of measurement services developed within NIST, in cooperation with other Government agencies or private groups, or in cooperation with international metrology organizations." Paul Hale and Dylan Williams of EEEL and Jack Wang of ITL's Statistical Engineering Division were named as co-winners. The team was cited for "providing a world-first method for simultaneously calibrating high-speed electrical test equipment in both time and frequency domains with point-by-point uncertainty analysis, and for the first time making waveform calibrations trace-

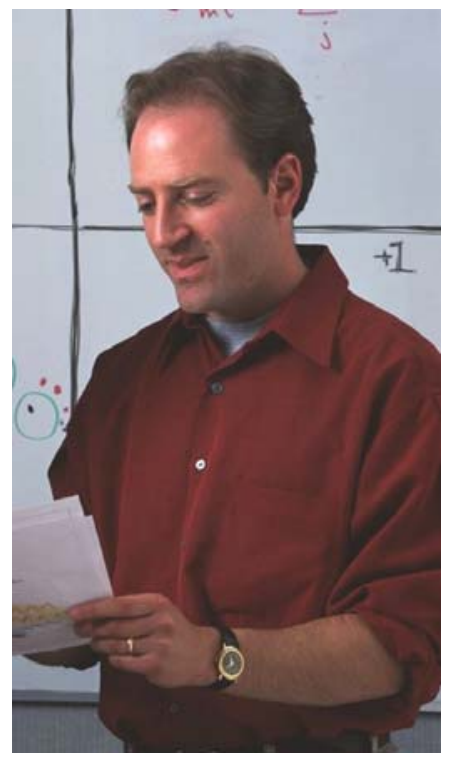

Andrew Dienstfrey was a co-winner of NIST's Allen V. Astin Measurement Science Award.

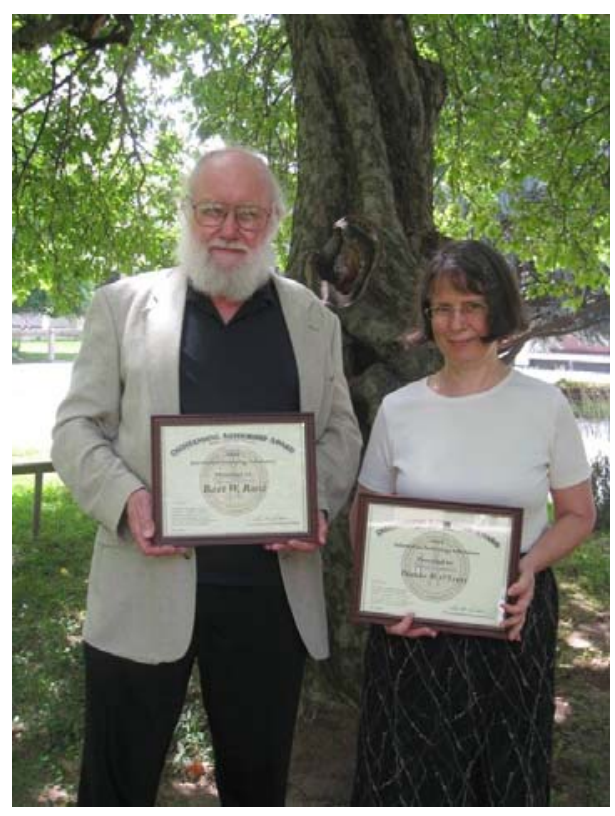

Bert Rust and Dianne O'Leary received ITL's Outstanding Journal Paper Award for 2009. able to fundamental physics."

o ITL Outstanding Journal Paper. Bert Rust and Dianne O'Leary were honored with the 2008 ITL Outstanding Journal Paper Award for their article "Residual periodograms for choosing regularization parameters for illposed problems." The paper appeared in the journal Inverse Problems, Volume 24, Article Number 034055 (May 2008). The award citation reads "For notable scientific research and exposition in the field of inverse problems as applied to measurement science."

\section{Best Contribution Awards}

o CIRP Conference on Modeling of Machining Operations. A paper presented by Timothy Burns of MCSD at the 12th CIRP (The International Academy for Production Engineering) Conference on Modeling of Machining Operations, in San Sebastian, Spain, May 7-8, 2009 was one of a few submissions selected for publication in the international journal Machining Science and Technology. The paper, "Dynamic properties for modeling and simulation of machining: An update of results from the NIST pulse-heated Kolsky bar," discussed some significant implications for finiteelement simulations of new NIST material response measurements on stress vs. strain response under conditions of extremely rapid heating of carbon steel in high-speed machining. Co-authors on the paper were Steven Mates of NIST/MSEL, Richard Rhorer and Eric Whitenton of NIST/MEL, and Debasis Basak, of Orbital Sciences Corporation. 
o IEEE Personal, Indoor and Mobile Radio Communications Symposium. A paper co-authored by John Hagedorn and Judith Terrill of MCSD received one of the three Best Paper awards at the 20th Personal, Indoor and Mobile Radio Communications Symposium (PIMRC) held in Tokyo, Japan on September 13-16, 2009. PMIRC is one of the major IEEE Communications Society conferences; some 650 papers were presented there this year. The cited paper,

"A Statistical Path Loss Model for Medical Implant Communication Channels," describes a new model to characterize RF path loss from wireless medical implants. The paper was presented by co-author Kamran Sayrafian, ITL Pervasive Information Tech-

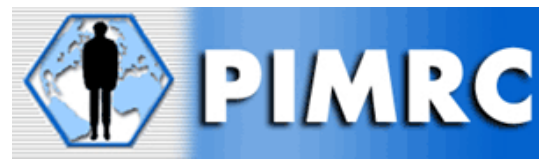
nology Program Manager. Wen-Bin Yang of the ITL Advanced Networking Technologies Division and Kamya Yekeh Yazdandoost of the National Institute of Information and Communications Technology in Yokosuka, Japan were also co-authors.

o Scientific Discovery through Advanced Computing (SIDAC) Conference. A short movie entitled "Simulation of Non-Newtonian Suspensions: Shear Thinning Case" developed by MCSD's Scientific Applications and Visualization Group in collaboration with NIST/BFRL scientists was chosen to receive an OASCR Award at the 2009 Scientific Discovery through Advanced Computing (SciDAC) Conference held in San Diego on June 14-18, 2009. The SciDAC Conference brings together about 350 scientists participating in DOE's SciDAC Program along with other prominent

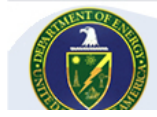
SciDAC Scientific Discovery through Advanced Computing researchers from the computational science community. Named for DOE's Office of Advanced Scientific Computing Research, OASCRs were awarded to 10 entries at the conference's Electronic Poster and Visualization Night session. The NIST entry illustrates how suspensions such as concrete or paint react as strain is applied. The movie was produced by William George, Steven Satterfield, John Hagedorn, Marc Olano and Judith Terrill of MCSD, along with Nicos Martys of BFRL's Materials and Construction Research Division. This is the second OASCR award garnered by the NIST team.

\section{Other Significant Recognition}

o Beichl Named Editor-in-Chief of Scientific Computing Magazine. Isabel Beichl of MCSD was selected to serve as Editor-in-Chief of Computing in Science and Engineering (CiSE), a joint publication of the IEEE Computer Society and the American Institute of Physics. CiSE is a bimonthly publication that combines peer-reviewed articles, columns, tutorials, and book reviews in a magazine format. According to its statement of purpose, CiSE “aims to support and promote the emerging discipline of computational sci-

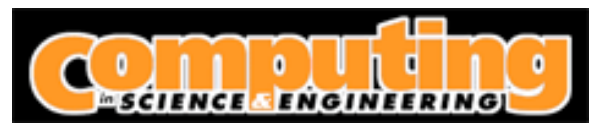
ence and engineering and to foster the use of computers and computational techniques in scientific research and education." Beichl's appointment as Editor-inChief, which was made by the IEEE Computer Society Board of Governors, is for a two-year term which began in January 2009. 


\section{Part II}

\section{Features}

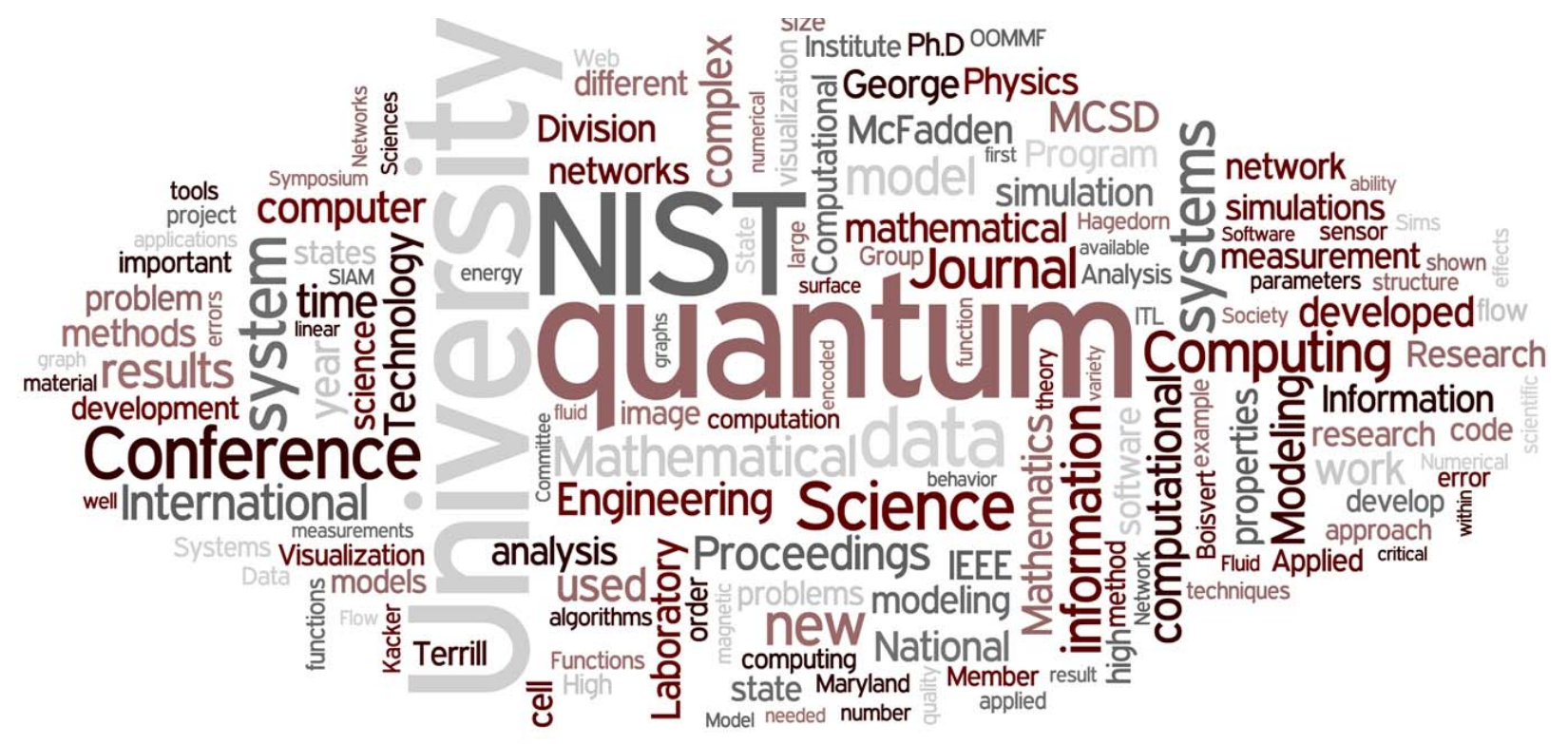




\section{Restrictions on Transversal Encoded Quantum Gate Sets}

During the last few decades, the theory of computation has gradually been expanded to encompass the possibility of quantum-mechanical effects in information processing. Quantum computers, as machines capable of exploiting such effects are called, have sparked considerable interest because it appears that they should be able to efficiently solve certain classically intractable problems. A working quantum computer has yet to be built, however, due to the extreme difficulty of precisely controlling complex quantum systems.

The accuracy required to implement many interesting quantum algorithms is daunting, but this accuracy need not be achieved directly in the hardware. As the theory of quantum computation has matured, a variety of software approaches have been developed to mitigate the effects of hardware imperfections. The foremost of these is quantum error correction, which, as in the classical case, relies on encoding the data of interest using a suitable code. Since the most practical quantum codes cannot reliably detect large numbers of errors, it is also important that encoded gates (operators used to transform encoded data) be designed to limit the spread of errors. One of the primary techniques for achieving this is the use of transversal operators.

Transversal operators provide an exceptionally simple and robust way of controlling the spread of errors. Thus, ideally, all gates necessary for quantum computation would be implemented transversally. However, the set of encoded gates that can be implemented transversally is determined by the quantum code employed, and despite substantial effort, no nontrivial quantum code has ever been found for which all encoded gates could be implemented transversally. This observation has led to a long-standing conjecture that transversal encoded gate sets cannot be universal. Recently, we have shown this conjecture to be true. A universal, transversal encoded gate set is incompatible with the most basic ability to detect errors.

\section{Bryan Eastin}

Like early classical devices, the first quantum computers will almost certainly be constructed from rather unreliable components. Consequently, strategies for managing errors are likely to be central to the operation of these machines. While classical error management is well understood, however, the requirements of quantum computing introduce new complexities that have forced researchers to reconsider the problem. It was, for example, not initially clear that the error correction of quantum data was even possible. Theoretical work on error management thus has an important role to play in the construction of a quantum computer. Indeed, in the last few years the gap between the error rate that is experimentally achievable and that which is proven to be sufficiently small as to allow for quantum computation has been narrowed as much by advances in theory as by experimental progress. The broader goal of this project is to contribute to the effort to build a quantum computer by improving our understanding of various strategies for managing errors, particularly quantum error correction and fault tolerance.

As in the classical case, quantum errors are rendered detectable by encoding the system of interest into a subspace of a larger, typically composite, system. A quantum code simply specifies which states of a quantum system correspond to which logical (encoded) information states. Errors that move states outside of the logical subspace can be detected by measuring the projector onto that subspace. Of course, not all errors can be detected; for any nontrivial code there are operators that act in a nontrivial way within the logical subspace. Most commonly, quantum codes are designed to permit the detection of independent, local errors and, as a consequence, are incapable of detecting some errors that affect many subsystems.

For quantum computation it is necessary not only to detect errors but also to apply operators (gates) that transform the logical state of the code. Even when error processes are local and independent, however, the operations entailed in computing can generate correlated errors from uncorrelated ones. Thus, for error detection to be effective, it is important that the logical operators employed during a quantum computation be designed to limit the spread of errors. It is particularly important that operators do not spread errors within code blocks, where a block of a quantum code is a collection of subsystems that undergoes error detection independently of all other subsystems. Managing the spread of errors is the subject of the theory of fault-tolerant quantum computing. One of the primary techniques of this theory is the use of transversal encoded gates.

Roughly, an operator is transversal if it couples no subsystem of a code block to any but the corresponding subsystem in another code block. (See Figure 1 for an illustration and a more detailed description.) Transversal operators are inherently fault tolerant. They can spread errors between code blocks, thereby increasing the number of locations at which a code block's error might have originated, but, since errors on different code blocks are treated independently, the total number of errors necessary to cause a failure is unchanged. This is in contrast to non-transversal operators, where, for example, an encoded gate coupling every subsystem in a code block might conceivably convert an error 


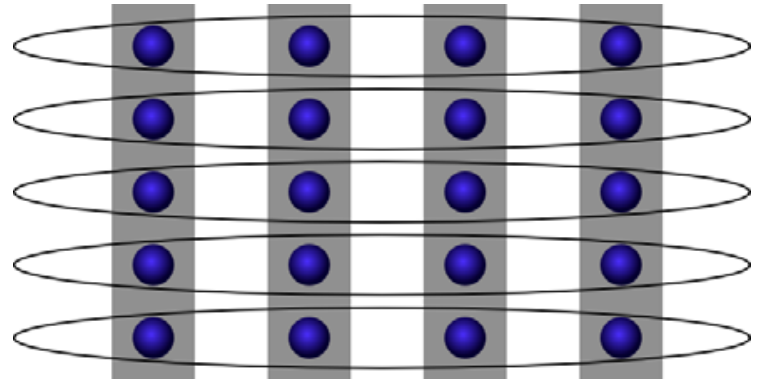

Figure 1. Illustration of a transversal partitioning of a quantum code. The blue spheres represent quantum subsystems, which are arranged into four code blocks, each identified by a gray rectangle. The ellipses divide the code into transversal parts, that is, parts containing one subsystem from each code block. Given such a transversal partitioning, a transversal quantum gate is one that only couples subsystems in the same transversal part.

on a single subsystem into an error on every subsystem in the block.

In view of the above, it would be highly desirable to carry out quantum computations exclusively using transversal encoded gates. To allow for arbitrary computation, it is necessary that the set of gates employed be universal, that is, that it be capable of implementing (through composition) any encoded operator on the logical state space to arbitrarily high accuracy. In spite of substantial effort, however, no gate set for a nontrivial quantum code has yet been found that is both universal and transversal. Consequently, a longstanding question in quantum information theory is whether there exist nontrivial quantum codes for which all logical gates can be implemented transversally.

For an important subclass of quantum codes known as stabilizer codes, this question was recently answered in the negative. Zeng et al. [1] showed that transversal unitary operators are not universal for stabilizer codes on two-level subsystems (qubits); the companion result for the case of $d$-level subsystems (qudits) was proven by Chen et al. [2]. In reference [3], Emanuel Knill and I used a different approach to prove a stronger version, showing that the ability of a quantum code to detect arbitrary errors on component subsystems is incompatible with the existence of a universal, transversal, and unitary encoded gate set.

The proof of this result is too involved to be included here, but it can be motivated as follows. Consider a quantum code such that

$$
\bar{Z}_{\theta}=\mathrm{e}^{\mathrm{i} \theta Z} \otimes \mathrm{e}^{\mathrm{i} \theta Z} \otimes \ldots \otimes \mathrm{e}^{\mathrm{i} \theta Z}=\prod_{j} \mathrm{e}^{\mathrm{i} \theta Z_{j}}
$$

is a valid logical gate for any $\theta$. For sufficiently small $\theta$

$$
\bar{Z}_{\theta} \approx \prod_{j}\left(I+\mathrm{i} \theta Z_{j}\right) \approx I+\mathrm{i} \theta \sum_{j} Z_{j}
$$

where each $Z_{j}$ acts on a single subsystem. Thus, the code cannot detect arbitrary single-subsystem errors since otherwise it would sometimes mistake the encoded gate $\bar{Z}_{\theta}$ for an error. There are several flaws in this argument, but using the theory of Lie groups it can be generalized and made rigorous, as we demonstrate in [3].

Our result is fairly broadly applicable. The proof makes no assumptions about the dimensions of the quantum subsystems beyond requiring that they be finite. The quantum system encoded is assumed only to be nontrivial, that is, to have dimension greater than one. The precise structure of the quantum code and its initialization state are left unspecified. Nonetheless, like any impossibility proof, ours can be circumvented by violating the assumptions.

In [3] we discuss several possible circumventions, some of which might make interesting projects for future research. While further study could be fruitful, however, it is important to note that the success of quantum computing does not depend on the existence of universal, transversal encoded gate sets. There are well-known schemes for achieving fault-tolerant universal quantum computation using ancillary states, where the preparation, testing, and coupling of the ancillary states makes extensive use of measurements and classical feed-forward. Thus, we have ruled out a desirable path to quantum computing but not an indispensable one.

\section{References}

[1] B. Zeng, A. Cross, and I. L. Chuang, Transversality versus universality for additive quantum codes, arXiv:0706.1382 (2007).

[2] X. Chen, H. Chung, A. W. Cross, B. Zeng, and L. Chuang, Subsystem stabilizer codes cannot have a universal set of transversal gates for even one encoded qudit, arXiv:0801.2360, Physical Review A 78 (2008), 012353.

[3] B. Eastin and E. Knill, Restrictions on Transversal Encoded Quantum Gate Sets, arXiv:0811.4262, Physical Review Letters 102 (2009), 110502.

\section{Participants}

Bryan Eastin and Emanuel Knill 


\section{High Precision Calculations of Fundamental Properties of Few- electron Systems}

Computational scientists at MCSD and Indiana University have achieved record levels of accuracy in the development of computational methods for determining fundamental properties of three electron atoms. Their recent result for the nonrelativistic energies for four excited states of the lithium (Li) atom represents the highest level of accuracy ever reached $\left(10^{-9}\right.$ hartree) in atomic quantum computations with more than two electrons. Familiar chemical electron affinities and ionization energies involve these nonrelativistic energies, so today we may be witnessing again a situation in which quantum mechanical calculations yield more accurate determinations of these fundamental properties than can be measured experimentally.

\section{James Sims}

NIST has long been involved in supplying critically evaluated data on atomic and molecular properties such as the atomic properties of the elements contained in the Periodic Table and the vibrational and electronic energy level data for neutral and ionic molecules contained in the NIST Chemistry WebBook. Fundamental to this endeavor is the ability to predict, theoretically, a property more accurately than even the most accurate experiments. Our goal is to accomplish this for fewelectron atomic and molecular systems.

Impressive advances have been made in the study of atomic and molecular structure, at both the experimental and theoretical levels. For atomic hydrogen and other equivalent two-body systems, exact analytical solutions to the nonrelativistic Schrödinger equation are known. It is now possible to calculate essentially exact nonrelativistic energies for atomic helium (He) and other three-body systems as well. Even for properties other than the nonrelativistic energy, the precision of the calculation has been referred to as "essentially exact for all practical purposes" [1], i.e., well beyond what can be achieved experimentally. Notwithstanding this theoretical advance, the data (experimental and theoretical) on atomic energy levels remains scarce, especially for highly ionized atoms. On the theoretical side, the availability of high precision results tails off as the state of ionization increases, not to mention higher angular momentum states. In addition, atomic anions have more diffuse electronic distributions, and therefore represent more challenging computational targets. On the diatomic molecular side, the presence of two fixed nuclei rather than one makes the integrals harder, hence exact analytical solutions to the molecular nonrelativistic Schrödinger equation are known (in the Born-Oppenheimer approximation) only for the one-electron $\mathrm{H}_{2}{ }^{+}$ion and other equivalent one electron systems. The challenge for computational scientists is to extend the phenomenal accomplishments on atomic helium to three, four, and more electron atomic states and to molecular systems.

Dihydrogen, $\mathrm{H}_{2}$, is the first molecule whose dissociation energy was correctly predicted by quantum mechanical calculation (1968) before this same quantity was measured reliably by experiment. Today we may be witnessing again a situation in which quantum mechanical calculations yield more accurate determinations of this fundamental property than can be measured experimentally. Very high precision approximations are now available for molecular hydrogen (a two electron system) as a result of our calculated Born-Oppenheimer (BO) energies of ${ }^{1} \Sigma_{g}^{+}$states of $\mathrm{H}_{2}$. With an error of 1 in the 13th digit, our results [2] were the most accurate energy values ever obtained for a molecule of that size, 100 times better than the best previous value, calculated or experimental.

The $\mathrm{BO}$ energies are used to provide the $X^{1} \Sigma_{g}{ }^{+}$potential energy curve which is critical to determining, theoretically, the dissociation energy $\mathrm{D}_{0}$ of $\mathrm{H}_{2}$, as shown in Figure 2. Recently [3], a new theoretical bond dissociation energy was obtained for the hydrogen molecule by fitting the $\mathrm{BO}$ potential needed to our computed energies over 47 inter-nuclear distances from 0.4-6.0 bohr. The resulting dissociation energy is accurate to 9 significant digits, two orders of magnitude better than the best previous experimental result.

While these results are important, the methods used are perhaps equally so. The calculation requires solving an approximation of the Schrödinger equation, a central equation in quantum mechanics. Its solution can be approximated as an infinite sum, each additional term contributing a bit more to the accuracy of the result. For all but the simplest systems or a relative handful of terms, however, the calculation rapidly becomes impossibly complex. While very precise calculations have been done for systems of just three components such as helium (nucleus and two electrons), we were the first to attain such precision for $\mathrm{H}_{2}$, a system with two nuclei and two electrons.

To make the problem computationally practical, we merged two earlier algorithms - one which has advantages in ease of calculation, and one which more rapidly achieves accurate results - into a hybrid, the Hylleraas-Configuration Interaction (Hy-CI) method, with some of the advantages of both. Our most recent work has shifted from the two-electron molecular case to the three-electron atomic case, using this hybrid 


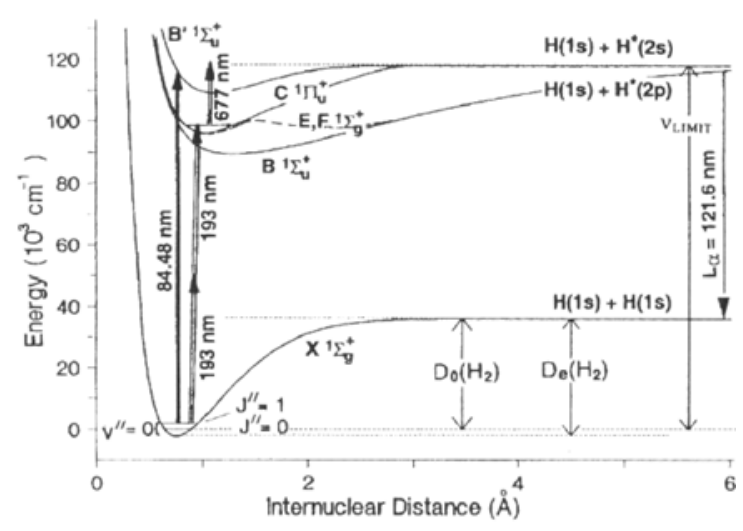

Figure 2. Potential energy curves of $H_{2}$ showing transitions from the ground state to the $B^{\prime l} \Sigma_{u}{ }^{+}$state in photodissociation.

method to extend the high precision accomplishments on atomic helium to atomic lithium, computing four excited states of the lithium atom to two orders of magnitude greater than has been done before. In calculations published in Physical Review A [4], we showed how Hy-CI calculations can achieve sub-nanohartree accuracy for the three-electron lithium atom.

There are two reasons why sub-nanohartree nonrelativistic energies are desirable. First, the physical energies of interest, e.g., a transition energy or familiar chemical ionization potentials and electron affinities, involve these nonrelativistic energies. So the nonrelativistic energies must be calculated very accurately to guarantee the accuracy of downstream results. Second, when a nonrelativistic energy is obtained, a wave function is also obtained which can be used to compute other physical properties like oscillator strengths, electric polarizabilities, Fermi contact terms, or nuclear mass polarization corrections. Quantum theory tells us that a first order error in the wave function manifests itself as a second order error in the energy. This can be understood from the fact that the energy is a sum of kinetic and potential energy contributions, so there can be cancellation of errors between the two energy contributions leading to a precision in the energy determination that does not carry over to other properties. Hence the drive for high accuracy calculations.

All results reported in our paper were obtained using quadruple precision $(\sim 30+$ digits) floating point subroutines written in Fortran 90, and required removing several bottlenecks to highly accurate $\mathrm{Hy}-\mathrm{CI}$ calculations: the three electron triangle integrals [5] and treating the remaining three-electron kinetic energy and nuclear attraction integrals [6]. We solved the secular equation, a generalized eigenvalue problem, using our own portable parallel inverse iteration solver. The assembly of the necessary matrices was also parallelized since that allows the total memory needed to be spread across the processors and eliminates a need to communicate matrix elements between processors. For a 17,190 term wave function we achieved a 30 -fold speedup on 32 processors for the order $N^{3}$ step running on the NIST cluster of 411 Intel Xeon, Intel Pentium 4, and AMD Opteron processors running Red Hat Linux.

In going from $\mathrm{He}$ (two electrons) to $\mathrm{Li}$ (lithium, three electrons) to Be (beryllium, four electrons), the high precision calculation situation degrades to the point that already at four electrons $(\mathrm{Be})$ there are no calculations of the ground or excited states with an error of less than $10^{-6}$ a.u. We are currently building the infrastructure for a calculation on the ground state energy level of the Be atom which has involved our dealing with four-electron integrals [7]. The beryllium calculation will determine whether really accurate calculations on atoms with $N \geq 4$ become a reality.

\section{References}

[1] G. W. F. Drake and W. C. Martin, Ionization Energies and Quantum Electrodynamic Effects in the Lower 1sns and 1snp Levels of Neutral Helium $\left({ }^{4} \mathrm{He}\right.$ I), Canadian Journal of Physics 76 (1998), pp. 679-698.

[2] J. S. Sims and S. A. Hagstrom, High Precision Variational Calculations for the Born-Oppenheimer Energies of the Ground State of the Hydrogen Molecule, Journal of Chemical Physics 124 (2006), 094101.

[3] K. Piszczatowski, G. Lach, B. Jeziorski, M. Przybytek, J. Komasa, and K. Pachucki, Theoretical Determination of the Dissociation Energy of Molecular Hydrogen, $J$. Chemical Theory and Composition, in review.

[4] J. S. Sims and S. A. Hagstrom, Hy-CI Study of the $2{ }^{2}$ S Ground State of Neutral Lithium and the First Five Excited ${ }^{2}$ S States, Physical Review A 80 (2009), 052507.

[5] J. S. Sims and S. A. Hagstrom, Mathematical and Computational Science Issues in High Precision Hy-CI Variational Calculations I. Three-electron integrals, Journal of Physics B 37 (2004), pp. 1519-1540.

[6] J. S. Sims and S. A. Hagstrom, Mathematical and Computational Science Issues in High Precision Hy-CI Variational Calculations II. Kinetic Energy and Nuclear Attraction Integrals, Journal of Physics B 40 (2007), pp. 1575-1587.

[7] J. S. Sims and S. A. Hagstrom, Mathematical and Computational Science Issues in High Precision Hy-CI Variational Calculations III. Four-electron integrals, in process.

\section{Participants}

James Sims. Stanley Hagstrom (Indiana University). 


\section{Characterization of Electromagnetic Metafilms}

Increasing reliability and control of nanometer-scale fabrication has created new possibilities for engineering materials with novel optical scattering properties. These devices will have tremendous impact in a variety of commercial, defense-related, and scientific applications such as magneto-optic storage, microantenna arrays, and integrated optical circuits. Metafilms are novel two-dimensional structures in which microscatterers are arranged as a planar lattice. The result is a new class of flexible, subwavelength coatings that exhibit strong EM scattering signatures. Novel applications proposed for metafilms include controllable "smart surfaces," microscale cavity resonators, novel waveguiding structures, and biomedical devices. Specialized computational tools capable of accurately guiding design parameters are essential for successful development of such devices. This past year NIST scientists made significant contributions to metafilm research by comparing a variety of theoretical predictions with a new high-accuracy simulation of welldefined vector EM scattering problems.

\section{Andrew Dienstfrey}

Metamaterials comprise one class of nano-scale electromagnetic (EM) scattering structures that have been the subject of ongoing study at NIST. A metamaterial consists of a three-dimensional lattice of tailor engineered scatterers embedded in a uniform substrate, the later referred to as "free-space." The scatterers exhibit geometry and composition such that their size is a fraction of a wavelength of light in free-space, nevertheless their internal structures admit resonant scattering. The resulting tension between length scales suggests the possibility that EM characteristics of the more extreme resonant variety may be observed in a bulk homogenized sense. Over the past decade many theories have emerged to predict the homogenized propagation characteristics of metamaterials. Many of these theories predict effective medium properties with exotic "double-negative" or "left-handed" EM propagation character, hence their considerable interest for both applications and theory. Metafilms are 2-D analogs of metamaterials in which micro-scatterers are arranged as a planar lattice. In the following the metafilm is composed of magneto-dielectric spherical particles in a planar, square-lattice configuration as in Figure 3.

Generalized Sheet Transition Conditions. There is disagreement in the theoretical community on the "correct" structural form for modeling EM scattering by a metafilm. One approach models the metafilm scattering problem as a bulk metamaterial with a small effective

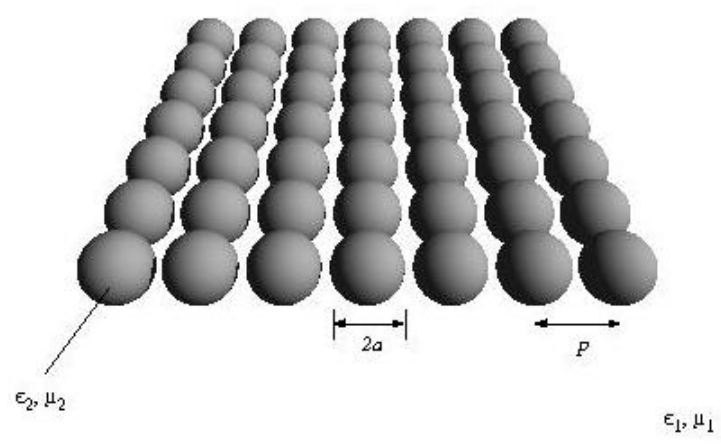

Figure 3. Metafilm composed of magneto-dielectric spherical particles with radius $a$, permittivity $\varepsilon_{2}$, permeability $\mu_{2}$, and lattice spacing $p$. $\left(a=10 \mathrm{~mm}, p=25.59 \mathrm{~mm}, \varepsilon_{2}=2 \varepsilon_{0}\right.$ and $\left.\mu_{2}=900 \mu_{0}\right)$.

thickness, $d$. With fixed $d$ reflection and transmission coefficients (obtained by either computation or measurement) may be inverted for effective material properties using classical thin-film inversion techniques. Alternatively, the NIST approach models a metafilm as an infinitesimal sheet of electric and magnetic polarizabilities. Electromagnetic scattering across such a sheet is described by generalized sheet transition conditions (GSTC)

$$
\begin{aligned}
\hat{\mathbf{z}} \times[\mathbf{H}] & =i \omega \varepsilon \alpha_{E} \cdot\left\langle\mathbf{E}_{t}\right\rangle+\hat{\mathbf{z}} \times \nabla_{t}\left\langle\alpha_{H}^{z z} H_{z}\right\rangle \\
\hat{\mathbf{z}} \times[\mathbf{E}] & =i \omega \mu \alpha_{E} \cdot\left\langle\mathbf{H}_{t}\right\rangle-\hat{\mathbf{z}} \times \nabla_{t}\left\langle\alpha_{E}^{z z} E_{z}\right\rangle \\
{\left[\varepsilon E_{z}\right] } & =-\nabla \cdot\left(\varepsilon \alpha_{E} \cdot\left\langle\mathbf{E}_{t}\right\rangle\right) \\
{\left[\mu B_{z}\right] } & =\nabla \cdot\left(\mu \alpha_{H} \cdot\left\langle\mathbf{H}_{t}\right\rangle\right)
\end{aligned}
$$

Here the subscript $t$ refers to transverse components, the polarizability tensors $\boldsymbol{\alpha}_{E}$ and $\boldsymbol{\alpha}_{H}$ are assumed diagonal, and the brackets $\langle\cdots\rangle$ and $[\cdots]$ represent the average and difference across the boundary $z=0$. Details can be found in [3]. In forward or predictive modeling, expressions for the tensors $\boldsymbol{\alpha}_{E}$ and $\boldsymbol{\alpha}_{H}$ are substituted into (1)-(4) and the reflection and transmission coefficients for the metafilm are computed. Alternatively, assuming the GSTC's, reflection and transmission coefficients may be inverted for the underlying tensors and comparisons can between theory and "experiment" can be performed.

This year we performed a detailed analysis to compare these competing theories. We observed that the artificial thickness parameter required of the metamaterial thin-film approach results in non-unique recovery of the effective medium properties, $\varepsilon=\varepsilon(d)$ and similarly for $\mu(d)$. An example of this non-trivial dependence is shown in Figure 4. As there is no a priori way to fix $d$, this non-uniqueness poses a fundamental problem for this approach. Although similar problems exist in classical thin film analysis, the 


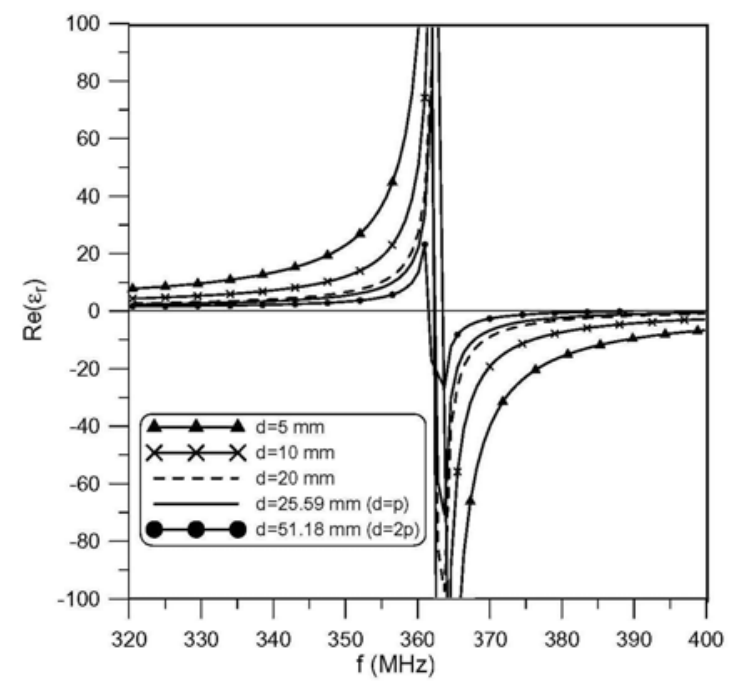

Figure 4. The retrieved effective permittivity of a metafilm modeled as a thin film exhibits a strong dependence on assumed layer thickness $d$. Same geometry and composition as above.

quantitative extent in the analysis of metafilms was previously unexplored. By contrast, the NIST approach retrieves unambiguous dielectric tensors that compare qualitatively and quantitatively with theoretical predictions. These results are documented in [2].

All Angle Total Reflection. The above analysis was performed assuming planewave incident light normal to the plane of scatterers, i.e. $\mathbf{k}=k \hat{\mathbf{z}}$. In an attempt to further validate the GSTC theory, scattering under offaxis illumination was computed and compared with the full angle-dependent GSTC theory. In the course of this work a surprising observation was made. Fullyresolved EM computations predicted that the lossless metafilm structure Figure 3 would exhibit angleindependent total reflection at specific resonant frequencies, for example see Figure 5. This strong, angleindependent scattering by a sub-wavelength structure was entirely unexpected. Subsequent analysis of the model polarizability tensors coupled with the GSTC's revealed that the NIST model also predicted this effect. The results of this analysis appear in [1].

Next Steps. We plan to continue investigating the GSTC model. In our study of angular-dependent scattering, detailed analysis of the GSTC's revealed surprising symmetries required for solvability. Briefly, for general planewave incidence all $\mathbf{E}$ and $\mathbf{H}$ fields may be expressed with respect to complex scattering coefficients in the canonical orthogonal frames defined by the scattering plane, i.e., the $\mathrm{R}_{t e}, \mathrm{R}_{t m}, \mathrm{~T}_{t e}$, and $\mathrm{T}_{t m}$ reflection and transmission coefficients. In doing this, the GSTC equations yield a linear system that may be solved either for scattering coefficients (R's and T's) in terms of polarizability tensors $\left(\boldsymbol{\alpha}_{E}, \boldsymbol{\alpha}_{H}\right)$ or conversely. In attempting to develop formulas for this inversion we discovered that the GSTC system is degenerate. Care-

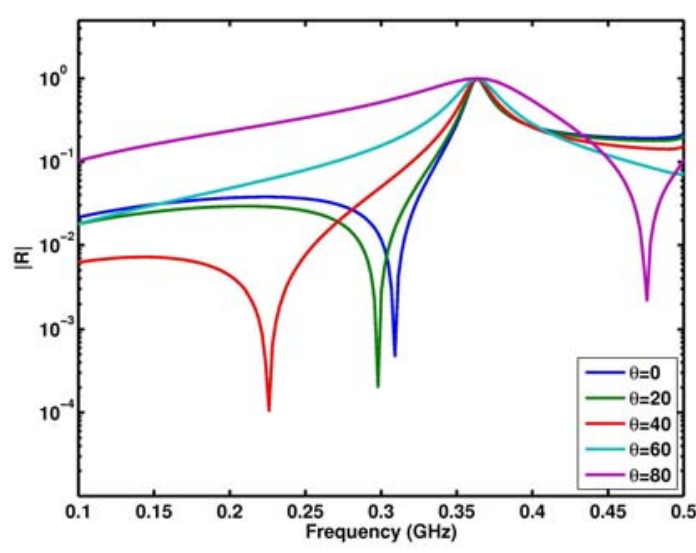

Figure 5. TE reflection coefficient shows all-angle, total reflection at resonance near $f \approx 3.5 \mathrm{GHz}$.

ful factorization of the linear system revealed four independent equations for the six unknown diagonal components of the polarizability tensors. What is more, we derived a solvability condition that physically corresponds to rotational symmetry of the scattering structure about the z-axis. For the spherical scattering structure depicted in Figure 3 this symmetry is reasonable. In fact, since our analysis assumed it from the outset this symmetry (solvability) constraint went unrecognized. That the GSTC's require such symmetry in general is unexpected, and suggests that fundamental terms may be missing from the equation. We also plan to explore applications for metafilms to problems in optical waveguiding. Finally, recent results have stimulated reanalysis of the computation of the Casimir force in the presence of metafilm scattering structures. We hope to consider this problem as well.

\section{References}

[1] J. A. Gordon, C. L. Holloway, and A. Dienstfrey, "A physical explanation of angle-independent reflection and transmission properties of metafilms/metasurfaces," IEEE Antennas and Wireless Propagation Letters 8 (2009), pp. 1127-1130.

[2] C. L. Holloway, A. Dienstfrey, E. F. Kuester, J. F. O'Hara, A. K. Azad, and A. J. Taylor, "A discussion on the interpretation and characterization of metafilms/metasurfaces: The two-dimensional equivalent of metamaterials," Metamaterials 3 (2009) pp. 100-112.

[3] E. F. Kuester, M.A. Mohamed, M. Piket-May, and C. L. Holloway, "Averaged transition conditions for electromagnetic fields at a metafilm," IEEE Transactions on Antennas and Propagation 51(10) (October 2003) pp. 2641-2651.

\section{Participants}

Andrew Dienstfrey. C. Holloway and J. Gordon (NIST EEEL). 


\section{Recovering from Physically Impossible Reconstructions in Blind Deconvolution}

A pervasive problem in imaging science is that most recorded imagery is blurred and degraded by noise. In many cases, the cause of the blur is only poorly known, and a reliable mathematical formula for the point spread function (psf) describing the blur is not available. Blind deconvolution seeks to reconstruct both the unknown psf $k(x, y)$, and the unknown sharp image $f(x, y)$, given only the recorded blurred image $g(x, y)$. This is a daunting problem fraught with serious mathematical difficulties, yet it is one that is of vital interest in numerous scientific contexts. Considerable effort continues to be directed at finding the right mathematical and computational formulation that would result in reliable blind deconvolution of large size scientific imagery.

\section{Alfred Carasso}

An important theoretical approach to blind deconvolution seeks to formulate that problem in terms of a variational principle. Using a-priori mathematical constraints on the unknown blur $k(x, y)$, and on the unknown sharp image $f(x, y)$, that approach seeks to obtain the pair $\{k, f\}$ as the unique minimizer of an appropriate cost functional. In recent years, several such linear and nonlinear functionals have been proposed. However, it may be anticipated that formulating a variational principle that would result in full deblurring of images with unknown blurs, remains a challenging problem in mathematical analysis. Indeed, the great diversity of possible image blurs, together with the difficulty of encapsulating the subtleties of the unknown sharp image into a mathematical statement that can serve as an a-priori constraint, make it unlikely that a useful all-encompassing principle can be found.

Recently, a highly efficient nonlinear variational approach was developed at the University of Bremen, [1]. This method is based on seeking that solution which differs from an educated first guess $\left\{k^{\dagger}(x, y)\right.$, $\left.f^{\dagger}(x, y)\right\}$ by the least amount in the $L^{2}$ norm. The blurred image $g(x, y)$ is used for $f^{\dagger}(x, y)$. The strength of this so-called Minimum Norm Solution (MNS) technique is that it can be formulated and calculated in Fourier space, and efficiently implemented using FFT algorithms. Indeed, almost instantaneous blind deconvolution ensues, even for $1024 \times 1024$ imagery.
The MNS method is critically examined in a recent paper by A. Carasso in the SIAM Journal on Applied Mathematics [2]. Fourier space behavior is used to identify and document three basic flaws in the MNS procedure. First, classical theorems of Bochner, Schoenberg, and Pólya are used to show that MNSdetected optical transfer functions (otf) are generally spurious, and are not characteristic functions. Such detected otfs must therefore correspond to non-physical psfs with negative values. Next, it is shown that only limited partial deblurring is theoretically possible in the MNS method, full deblurring being precluded by the MNS variational formulation. Lastly, it is shown that the MNS approach induces an erroneous relationship at high frequencies between the detected optical transfer function and the Fourier space reconstructed image. Each of these two objects becomes delusive at high frequencies. Unexpected spurious relationships between detected blurs and reconstructed images also occur in other variational deconvolution procedures.
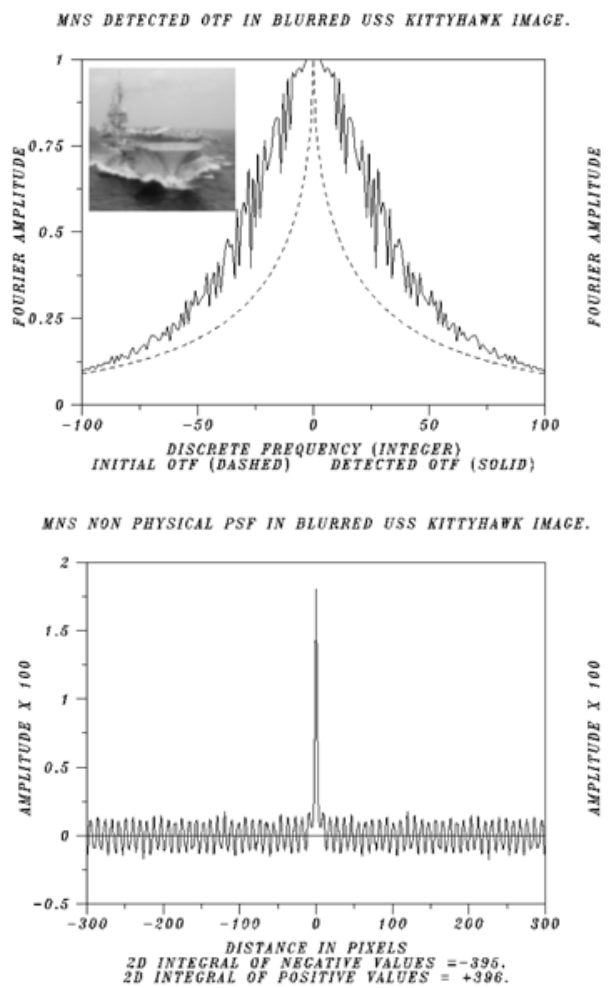

Figure 6. Failure of MNS approach in synthetically blurred $1024 \times 1024$ USS Kittyhawk image. Top: MNS detected otf (solid line) displays non-monotone behavior, at variance with classical theorems, and is not a characteristic function. This is confirmed in the bottom image showing that the defective otf above corresponds to a physically impossible, highly oscillatory psf $k^{\dagger}(x, y)$, exhibiting sustained negative values. While $k^{\dagger}$ integrates to 1 , its negative part integrates to -395 . 


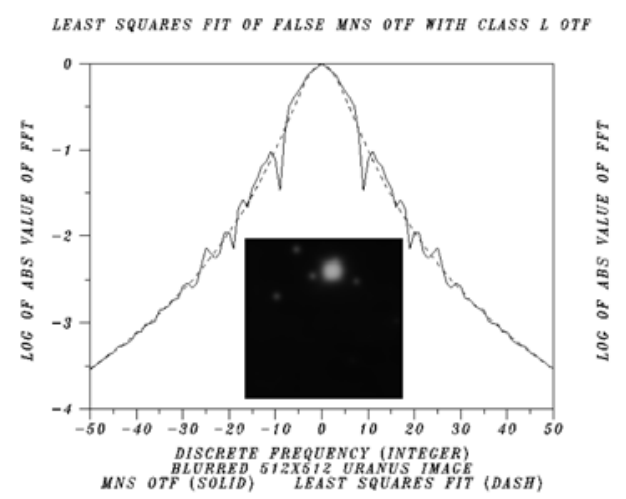

Figure 7. Blurred Uranus image experiment. Least squares fit of false MNS otf (solid line), with physically valid class $\boldsymbol{L}$ otf (dashed line) on restricted frequency interval $|\xi| \leq 50$. Dashed curve is postulated to be $p^{\text {th }}$ convolution root of true otf, with $p=2.5$. This tentative value can be readjusted by marching backwards in time.

SECB CONTINUATION IN URANUS MOONS IMAGE.

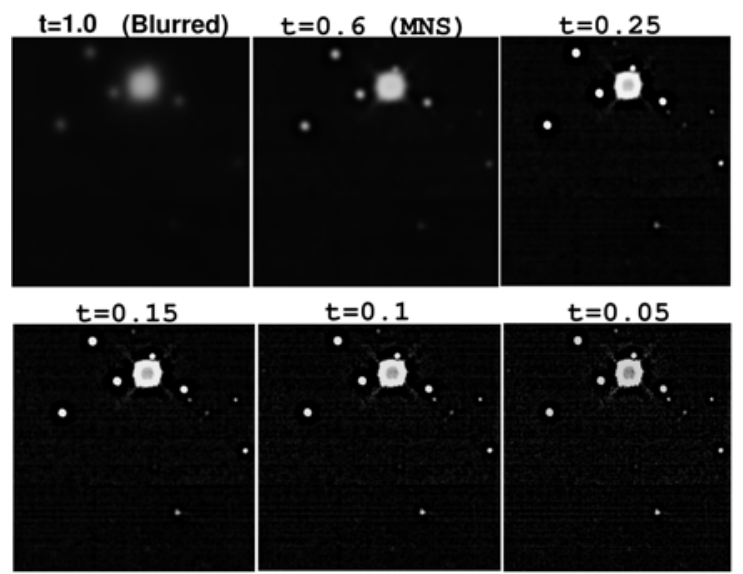

Figure 8. Blind deconvolution of Uranus image by marching backwards in time, from $t=1.0$ to $t=0$, using the class $\boldsymbol{L}$ otf detected in Figure 7. Partially deblurred MNS image corresponds to solution at $t=0.6$, and recovers 5 moons. However, Carasso's SECB methodology can continue solution to $t=0.05$, and recover 11 moons.

In the latter part of Carasso's SIAM paper [2], it is shown how to extract useful information from defective MNS reconstructions in the important special case of class $\mathbf{L}$ blurs, which includes Gaussians, Lorentzians, and other heavy-tailed densities, together with their convolutions. This intervention requires analytical considerations extraneous to the variational framework in [1], and involves several steps. First, the high frequency portion of the non-physical MNS-detected blur is discarded and a class $\mathbf{L}$ blur is associated with the low frequency part using least squares fitting. Next, this rectified blur is interpreted as the $p^{\text {th }}$ convolution root of the true blur for some postulated real number $p$ $\geq 2$. Infinite divisibility of this candidate true blur allows deconvolution to be performed in slow motion, by marching backwards in time in an associated parabolic equation, with the blurred image as data at $t=1$. Behavior of the image evolution as $t \downarrow 0$ can be monitored and used to readjust the value of $p$. Previously developed APEX/SECB methodologies [3] provide the necessary computational tools to make such an approach feasible.

Figure 6 illustrates one fundamental defect in the MNS method. In the top graph, the dashed curve depicts an educated guess $k^{\dagger}$ for the psf in the blurred USS Kitty Hawk image. The solid curve is the MNS detected otf. The oscillatory non-monotone behavior in that curve contradicts classical theorems of Schoenberg and Pólya on characteristic functions. This is confirmed in the bottom graph, which shows that the corresponding psf has sustained negative values extending over the entire range, and hence cannot represent a physical blur. Thus, the corresponding MNS image reconstruction is of questionable validity.

The next two figures illustrate the remarkable effectiveness of Carasso's intervention in blind restoration of a blurred image of Uranus and its moons. In Figure 7, the non-physical oscillatory MNS detected otf (solid line), restricted to the frequency range $|\xi|<$ 50 , is approximated by a class $\mathbf{L}$ otf (dashed curve), with the latter now assumed valid over entire frequency range. In Figure 8, the partially deblurred MNS reconstruction is shown to be equivalent to marching backwards in time from $t=1$ to $t=0.6$ in the governing associated parabolic equation. Such MNS deblurring reveals 5 Uranus moons. However, using the new class L otf from Figure 7, Carasso's SECB intervention can continue the backwards solution much further, to $t=0.05$, and can recover 11 Uranus moons. (Uranus has 27 known moons).

Coupled with Carasso's recovery methodology, MNS blind deconvolution is a valuable tool, one that can be used to verify the results of other blind deconvolution procedures. Future work will apply this technique to new state of the art scanning electron microscope imagery.

\section{References}

[1] L. Justen and R. Ramlau, A non-iterative regularization approach to blind deconvolution, Inverse Problems 22 (2006), pp. 771-800.

[2] A. S. Carasso, False characteristic functions and other pathologies in variational blind deconvolution. A method of recovery, SIAM Journal on Applied Mathematics 70 (2009), pp. 1097-1119.

[3] A. S. Carasso, The APEX method in image sharpening and the use of low exponent Lévy stable laws, SIAM Journal on Applied Mathematics 63 (2002), pp. 593618. 


\section{A Quality Pre-Processor for Biological Cell Images}

High content screening (HCS) has become a critical method for rapid large scale analyses in cell biology and drug discovery. HCS uses automated microscopes and sample preparation and includes the acquisition and analysis of cellular images without human intervention. Typical HCS-based experiments can involve the analysis of more than a million cells $[1,2,3]$. The process of automatically identifying objects and their boundaries in an image, known as image segmentation, is the most important part of analyzing biological image data. Consequently, many segmentation methods have been published, including histogram-based, edgedetection-based, watershed, morphological, and stochastic techniques. However, segmenting the same image with different methods can lead to different masks for a given cell, and hence different estimates of cell characteristics (e.g., area, perimeter, etc.). Accurate segmentation is one of the critical challenges facing high content screening. Therefore it is important to obtain a predictive, objective and efficient measure of the segmentation quality resulting from a particular method, on a cell-by-cell basis. Not all cells in the same image will lead to segmentations with the same accuracy. We have developed a simple, yet reasonably faithful, measure of the quality of an individual cell in an image to facilitate the automated choice of segmentation method and focus setting.

\section{Adele Peskin}

The goal of this project is to develop a quality index for the representation of a cell in a biological image. This index is determined on a cell-by-cell basis by assessing the characteristics of the edge of each cell. As such, it is different from other quality indices proposed previously which focus on overall image noise and distortion $[3,4]$.

Data Description. To define a quality index for each cell, we first examine a series of images and their ranges in quality, prepared by our collaborators in the
NIST Chemical Sciences and Technology Laboratory. These reveal the types of features associated with cell images acquired in practice. These images are of A10 rat smooth vascular muscle cells and $3 \mathrm{~T} 3$ mouse fibroblasts stained with Texas Red cell body stain. For each set, we compared images acquired under five sets of imaging conditions, combinations of low, medium, and high illumination, as well as with optimal and nonoptimal filters. Figure 9 provides a visual rendition of the five conditions for two example cells.

Quality Calculation. For each cell in an image, we look at pixel intensities within an isolated region containing the cell. We fit this distribution using a 3component Gaussian mixture via the EM (ExpectationMaximization) algorithm (background, edge region, cell) [6]. For each pixel intensity value between the mean of the background distribution and the mean of the edge distribution, we also calculate an average value for the magnitude of the gradient at that intensity. We consider the gridded data within a bounding box on the image that contains a single cell. At each pixel location, $p_{i j}$, with pixel intensity, $I_{i j}$, we find a local derivative of that intensity and its magnitude, using a Sobel mask, and then find an average gradient magnitude for each intensity value.

The average gradient magnitude curve, $G(I)$ vs. $I$, reflects two cell characteristics. The initial slope of the curve indicates the sharpness of the edge: the steeper the slope, the sharper the edge. The shape of this curve also indicates a feature of the cell edges. For high illumination settings with very sharp edges, the average gradient magnitude increases monotonically across the range from the mean of the background to the mean of the edge distribution. For the low and medium illumination images, the gradient increases, reaches a maximum, and then falls, as seen in Figure 10.

We find the 3-component pixel intensity distribution, find and smooth the gradient in the region of the cell edge, and then find the expected fraction of the edge that lies in the 2-pixel-length region outward from the edge point of maximum intensity gradient. As an
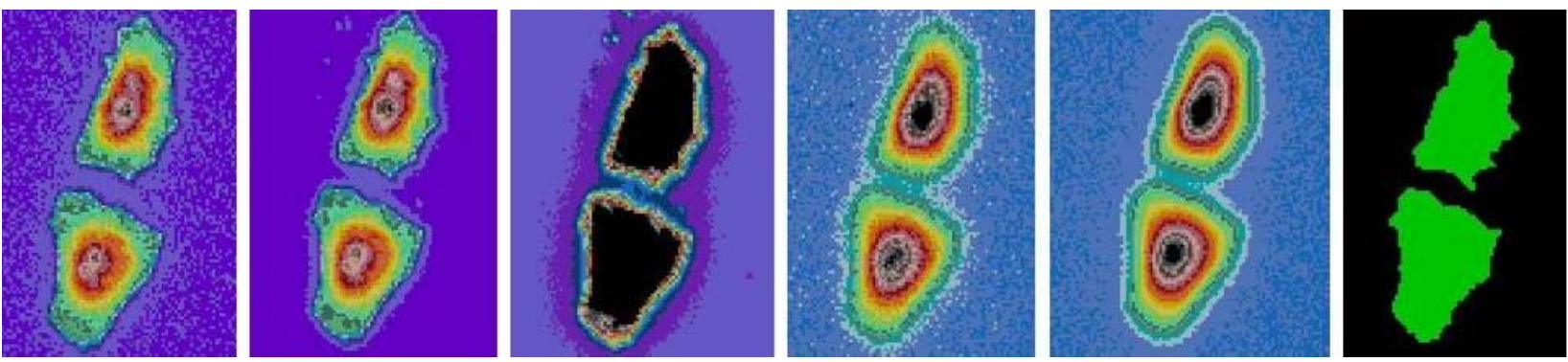

Figure 9. Five images acquired under different conditions, along with a ground truth mask (right). Individual pixel intensities are color-coded over the range in each image, to show differences in edge sharpness. 

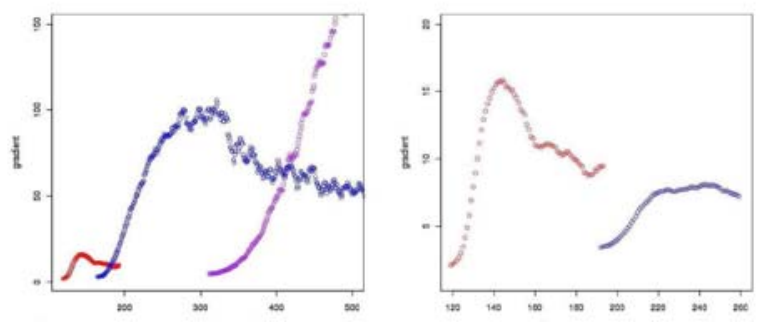

Figure 10. Left: Averaged magnitudes of the pixel gradient for low (red), medium (blue), and high (purple) illumination settings with the optimal filter. Right: For low, using optimal (red), vs. nonoptimal (blue) filters.

example, look at a cell with a very thin connection between the main part of the cell and pseudopodia (see Figure 11). These five cells have quality indices of $1.015,1.000,0.852,0.467$, and 0.429 respectively. Physically, that represents cell edges that cover from 2 to 4 pixel to pixel length distances.

Data Creation. We then synthetically altered cell image examples to examine the effects of different imaging parameters on the quality index. Figure 12 shows the effects of altering the spread of the background pixel intensities and of shifting the edge pixel intensities closer to the background pixel intensities.

Conclusions. The goal of our quality index is to provide an indication of the segmentation method that should be used to yield the highest accuracy in derived cell quantities (e.g., area, perimeter, etc.). Ideally, we would like to be able to distinguish between those segmentation methods that over- or under-estimate cell area from those that provide unbiased estimates of cell area, based on the quality of the edge of the cell. Because our quality index as defined here measures only edge clarity, we need to modify it to better capture cell image features. Our future work will includes a more thorough investigation of the properties of this quality index, particularly its relation to accuracy of cell area. We hope to use this quality index (modified to include perimeter to area ratios) to automatically select segmentation methods during rapid analysis of large numbers of biological images.

\section{References}

[1] V. C. Abraham, D. L. Taylor, and J. R. Haskins, High content screening applied to large-scale cell biology, Trends in Biotechnology 22 (2004), pp. 15-22 .

[2] K. A. Giuliano, J. R. Haskins, and D. L. Taylor, Advances in High Content Screening for Drug Discovery, Assay and Drug Development Technologies 1(4) (2003) pp. 565-577.

[3] X. Zhou and S. T. C. Wong, High content cellular imaging for drug development, IEEE Signal Processing 23(2) (2006), pp. 170-174.

[4] Z. Wang, A. C. Bovik, H. R. Sheikh, and E. P. Simoncelli, Image quality assessment: from error visibility to structural similarity, IEEE Transactions on Image Processing 13(4) (2004), pp. 600-612.

[5] Z. Wang and A. C. Bovik, A universal image quality index, IEEE Signal Processing Letters 9(3) (2002), pp 81-84.

[6] A. P. Peskin, K. Kafadar, A. M. Santos, and G. G. Haemer, Robust Volume Calculations of Tumors of Various Sizes, in 2009 International Conference on Image Processing, Computer Vision and Pattern Recognition (2009).

\section{Participants}

Adele Peskin. Karen Kafadar (Indiana University). Alden Dima (NIST ITL). Anne Plant, John Elliot, and Michael Halter (NIST/CSTL).
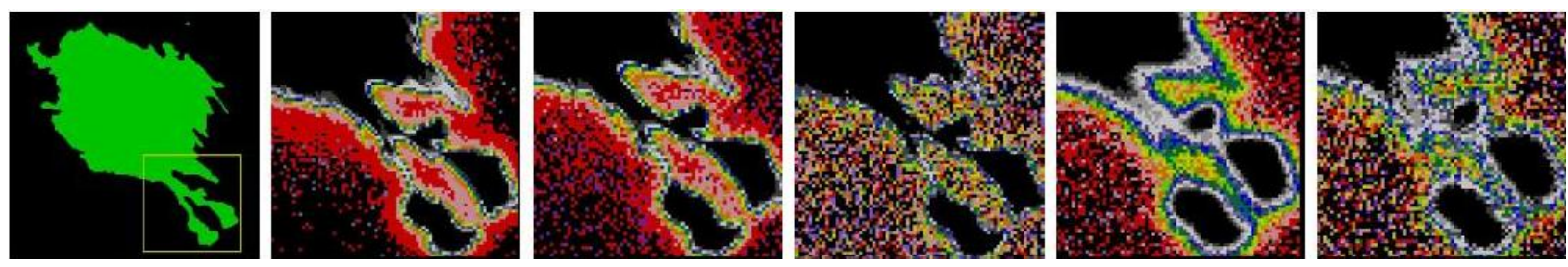

Figure 11. A cell mask and enlarged victures of the lower right hand marked section. in order of decreasing aualitv index.
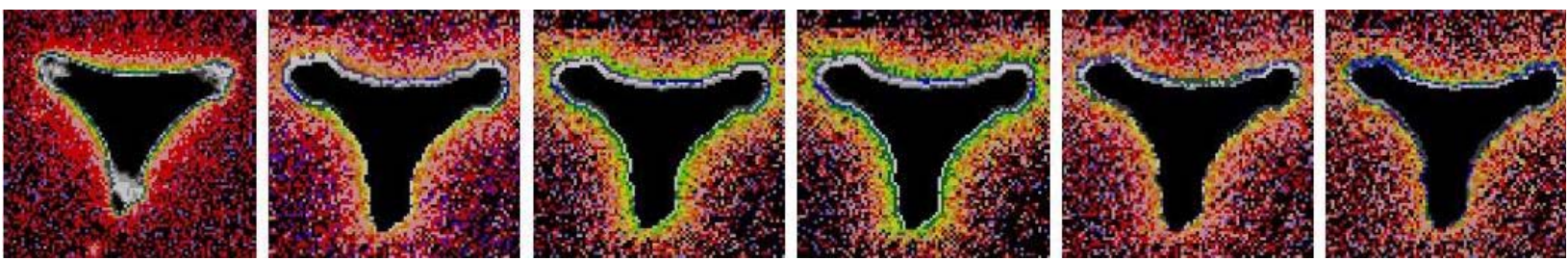

Figure 12. From left to right: (a) Original cell imaged with medium illumination, optimal filter. (b) Synthesized cell of the same type as (a), made as a test case. (c) and (d) Two cells identical except for the spread of the background pixels; standard deviations are 10 and 12 compared to original 5. (e) and (f) Two cells identical except that the edge distribution has been shifted from a mean of 225 to a mean of 215 and 205. 


\section{Computational Modeling of the Flow of Dense Suspensions}

\begin{abstract}
Understanding the flow properties of complex fluids such as suspensions is of technological importance, but presents significant theoretical challenges. Computational models of such systems require the tracking of boundaries between different fluid/fluid and fluid/solid phases, which can be extremely difficult. In our models we use methods from Dissipative Particle Dynamics and Smoothed Particle Hydrodynamics to simulate the flow of cement and concrete. By using these methods, we avoid the traditional difficulties in tracking boundaries by instead tracking individual mesoscopic scale particles. We have developed efficient parallel implementations of these methods, allowing us to simulate larger systems, with realistically shaped particles (instead of spherical particles), and for longer simulation times, than was previously possible. The results from our simulations have been validated against both theoretical results and results from physical experiments. Analysis of recent simulations has led to an advance in the conceptual understanding of the mechanisms that control the onset of flow in suspensions.
\end{abstract}

\section{William George}

While concrete may sound like a low tech material, modeling and predicting its rheological properties, that is, its physical flow properties, remains a great challenge. Concrete is actually a very complex fluid involving phenomena taking place over many length and time scales. For example, concrete is typically composed of cement (micrometer scale), sand (millimeter scale) and aggregates (centimeter scale). Clearly, no computer simulation can simultaneously include all these length scales, so we have developed a multi-scale approach linking phenomena at different length scales. For example the rheological properties of cement paste can be used as input to simulations of a mortar (cement paste and sand) and mortar properties as input into simulations of concrete (mortar and coarse aggregate). However, even at each representative length scale there can be considerable variation and other factors to consider such as the addition of chemical admixtures in cement paste, variation by over a factor of 100 in the size of aggregates, and shape variation of aggregates (rounded or crushed).

There is a wide body of literature on the rheology of suspensions. Unfortunately, most analytical results can only describe the properties of simple suspensions, most typically very dilute suspensions. Understanding the flow of more complex suspensions (e.g., dense suspensions, suspensions composed of particles with different size and shapes, etc.) represents a great challenge. Current prediction of the rheological properties

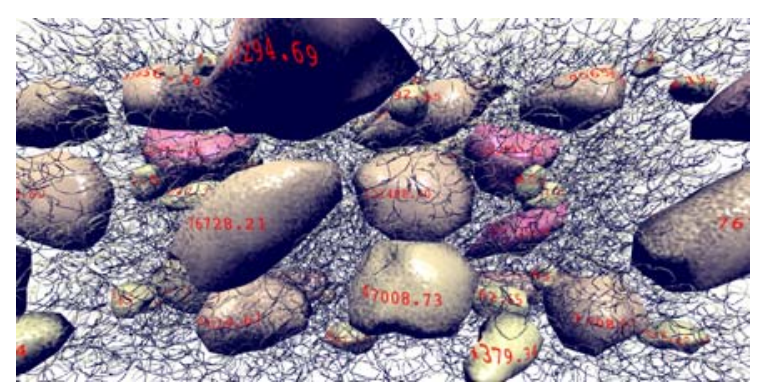

Figure 13. A snapshot of a simulation. Only the aggregates with the highest stresses are shown in this display, with the other aggregates shown as outlines. Actual stress values are painted on the surface of the aggregates.

of dense suspensions is usually based on semiempirical models such as the Kreiger-Doughtry equation which is used to predict the relative viscosity as a function of maximum packing fraction and knowledge of the intrinsic viscosity of a representative particle of the suspension. Rigid bodies having shapes like crushed aggregates are poorly accounted for by such approaches and there is no accepted theoretical understanding. Direct measurement of the rheological properties of such systems is itself a significant challenge, and there is no standard practice or database for validation of measurements.

As a result, we have turned to computational modeling of suspensions to better understand the flow of such systems and to give interpretation of measurements a better scientific basis. Modeling complex suspensions using computational fluid dynamics, while promising, remains a challenge because it is difficult to track boundaries between different fluid and solid phases. Thus, its practical applicability to modeling dense suspension remains in question.

Recently, a new computational method called dissipative particle dynamics (DPD) has been developed which holds promise for modeling such complex fluids. DPD has the advantage that it can naturally accommodate many boundary conditions while not requiring meshing (or remeshing) of the computational domain. On the surface, DPD looks very much like a molecular dynamics algorithm where particles, subject to inter-atomic forces, move according to Newton's laws. However, the particles in DPD are not atomistic, but instead are a mesoscopic representation of the fluid. The interactions between DPD particles are typically produced by three classes of forces: conservative, dissipative, and random. The conservative force is simply a central force, derivable from some potential. The dissipative force is proportional to the difference in velocity between particles and acts to slow down their relative motion. The dissipative force can be shown to produce a viscous effect. The random force (usually 

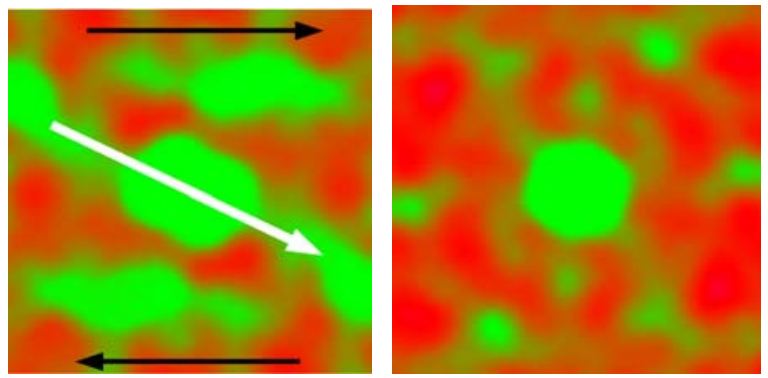

Figure 14. Left: This image illustrates the correlation of shear stress in a suspension. The green represents high stress regions and the red represents lower stress regions. The white arrow highlights the stress that is highly correlated in the "compression quadrant", that is, with aggregates being pushed together as we begin to strain the system. The black arrows indicate the direction of the applied shearing force. Right: Image is from the same simulation, showing the stress in the system after is has been sheared. Note the emergence of an ordered six-fold symmetry.

Gaussian noise) helps maintain the temperature of the system while producing some viscous effects. In order to maintain a well defined temperature consistent with a fluctuation-dissipation theorem, coefficients describing the strength of the dissipative and random forces must be coupled. We have adopted this approach as it has the potential of modeling rigid bodies with a wide variety of shapes.

In addition to this DPD-based model, which simulates suspensions with Newtonian fluid matrices, we have also implemented a Smoothed Particle Hydrodynamics (SPH) model of dense suspensions. SPH, which also incorporates a mesoscopic particle model similar to DPD, simulates suspensions with non-Newtonian fluid matrices. This is important because while cement paste has a Newtonian fluid matrix (water), mortar (cement + sand) and concrete (mortar + aggregates) have non-Newtonian fluid matrices. In non-Newtonian fluids, viscosity varies with the local shear rate.

In order to perform simulations on systems of sufficient size, density, and simulation time, we have developed highly parallel implementation of our models. This implementation, called QDPD, has run simulations efficiently using more than 2,000 processors. In these cases we often simulate systems consisting of over 1 million mesoscopic fluid particles and up to 10,000 aggregates. The output from these simulations includes the overall system stress over time, from which the viscosity can be computed, as well as periodic snapshots of the system that captures the location, orientation, and velocity of each of the system's aggregates. Post-processing of these snapshots generates an animation of the simulation which can be displayed in our immersive visualization system. One such simulation snapshot is shown in Figure 13, where only the high-stress aggregates are shown fully.

Through awards granted by the DOE INCITE program, we have been able to run simulations on Intrepid, the IBM Blue Gene/P machine at the Argonne National
Laboratory Leadership Computing Facility. Intrepid, as of November 2009, is 8th in the Top 500 supercomputing list ${ }^{1}$. In 2008 and 2009 we were granted (and used) 750,000 CPU-hours on Intrepid. (1 CPU-hour $=1$ hour of processing time on 1 Intrepid CPU). On Intrepid our simulations use 512, 1000, or 4096 CPUs, depending on the size of the system being simulated. For 2010 we have been awarded 2 million CPU-hours of compute time on Intrepid, which will allow us to complete more large simulations than in previous years.

We have made several improvements to QDPD in the past year. One is a complete re-write of the file input/output routines to use parallel $\mathrm{I} / \mathrm{O}$ to and from single files, rather than to per-process files. This greatly improves post-processing of QDPD output, especially when running on more than 1000 processors, and makes simulation restarts from checkpoints much simpler. We have also added the ability to change the simulation time step dynamically to avoid instabilities in the simulations without the need to stop and restart. This allows the simulator to find the largest time step that it can use safely, thus reducing the total time needed to complete a simulation.

In general, our simulations have been designed to explore the effects of particle size and shape distribution, the volume fraction of aggregates in the suspension, and other relevant system parameters, on the yield stress and viscosity of suspensions. These have led to a variety of very useful insights. For example, we discovered that, for dense suspensions, a previously unidentified physical mechanism plays a role in yield stress, which is a measure of the internal resistance to the startup of flow. This discovery was made by analyzing the pattern of contacts between the aggregates in snapshots from the simulation [1]. Figure 14 shows the location of high stress regions in these simulations before the aggregates begin to move (left image) and after (right image).

\section{References}

[1] N. Martys, D. Lootens, W. George, P. Hebraud, Contact and stress anisotropies in start-up flow of colloidal suspensions, Physical Review E 80 (2009), 031401.

\section{Participants}

W. George, S. Satterfield, J. Hagedorn, M. Olano, and J. Terrill. N. Martys (NIST BFRL). Virtual Cement and Concrete Testing Laboratory Consortium.

http://math.nist.gov/mcsd/savg/parallel/dpd http://math.nist.gov/mcsd/savg/vis/concrete

${ }^{1}$ http://www.top500.org/ 


\section{Visualization of Radio Frequency Propagation for Body Area Networks}

\begin{abstract}
A Body Area Network (BAN) is a network intended to be used in or around the human body. While this is an emerging field, networks of medical sensors are anticipated to be a primary application. Such sensors would either be attached to or implanted in the human body and would communicate wirelessly both within the body and to devices outside. For this technology to develop, greater understanding of radio frequency propagation through the human body is needed. In this project, we seek to provide such insight. This work is assisting IEEE standards committees to develop communications standards and will help RF engineers to design effective communication devices for BANs.
\end{abstract}

\section{John Hagedorn}

Body Area Networks (BANs) consisting of radioenabled wearable and implantable sensory nodes are poised to be a promising technology with novel uses in pervasive health information technology, such as smart pills for precision drug delivery, glucose monitors, blood pressure sensing systems, and eye pressure sensors for glaucoma patients. However, numerous challenges remain, including size, cost, energy source, sensing/actuator technology, and transceiver design. Some recent advances in microelectronics indicate that the technology to achieve ultrasmall and ultra low power devices is within reach.

For this technology to develop, greater understanding of radio frequency (RF) propagation through the human body is needed. In this project, we seek to provide such insight. In particular, we are providing technical support to IEEE standards committees to develop communications standards and to RF engineers who seek to design effective communication devices for BANs.

Because experimentation on human subjects is not feasible, RF propagation through the human body is being modeled using a 3D full-wave electro- magnetic field simulator. The software we are using is HFSS, a product of the Ansoft Corporation. Simulations are based on a 3D human body model which includes frequency dependent dielectric properties of $300+$ parts in a male body.

The output data produced by this simulation software is brought into our custom 3D immersive visualization system, which enables researchers to study the modeled RF propagation through direct interactions with the data. Figure 15 shows a visualization of a simulation of a transmitter implanted in the right forearm with color coding on the surface of the body based on signal loss.

Because communication with an implanted or wearable device can be done from any direction inside or outside the body, a true 3D environment is needed to capture, visualize and understand RF propagation to and from implants. The 3D immersive visualization platform that we have developed is shown in Figure 16. It includes several key components: three orthogonal screens that provide the visual display, motion tracked stereoscope glasses, and a hand-held motion tracked input device. The screens are large projection video displays that are placed edge-to-edge in a corner configuration. These three screens are used to display a single 3D stereo scene. The scene is updated based on the position of the user as determined by the motion tracker. This allows the system to present to the user a $3 \mathrm{D}$ virtual world within which the user can move and interact with the virtual objects. The main interaction device is a hand-held three button motiontracked wand with a joystick.

This virtual environment allows for natural interaction between experts with different backgrounds such as engineering and medical sciences. The user can look at data representations at any scale and position, move through data, change orientation, and control the elements of the virtual world using a variety of interaction techniques including measurement and analysis.

All of these capabilities are

Figure 15. Simulation of a transmitter implanted in the right forearm. Color denotes signal loss. extremely useful when studying 


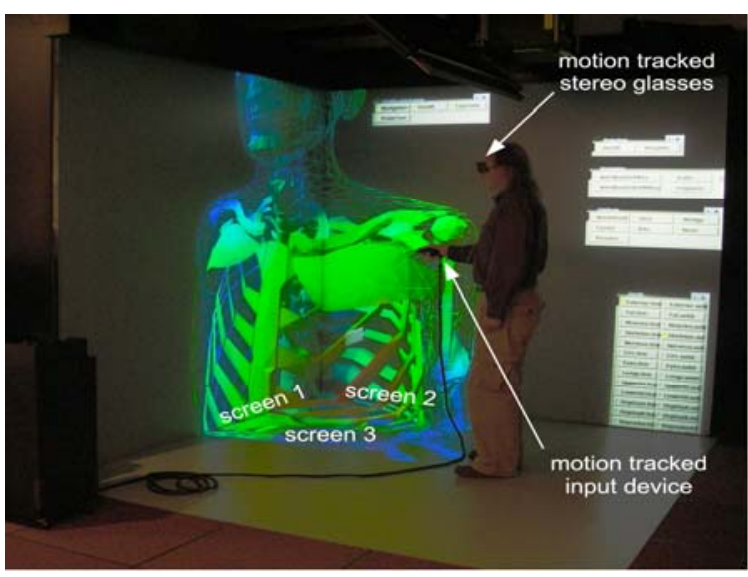

Figure 16. Our $3 D$ immersive visualization system. Several important components of the system are labeled and the user is shown interacting with $3 D R F$ data produced by our simulations.

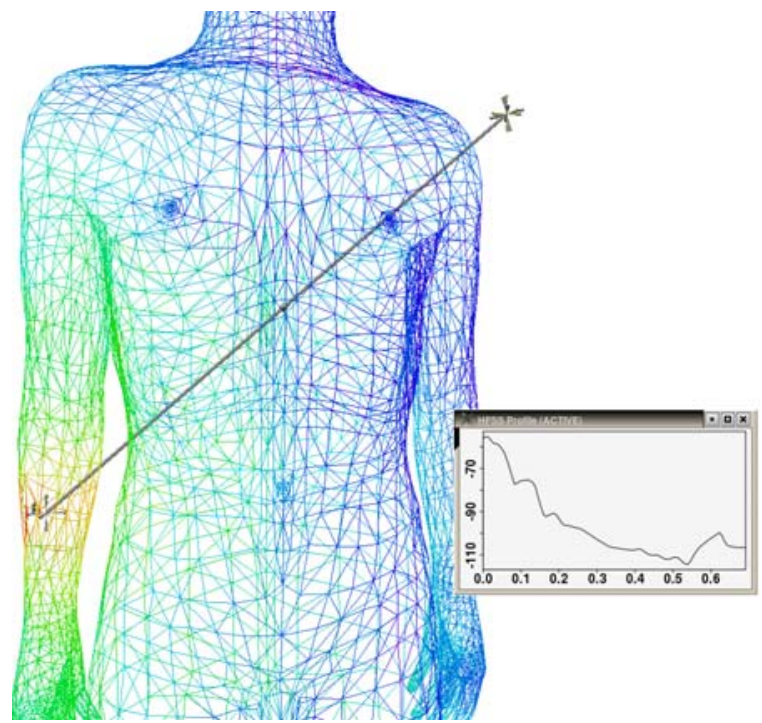

Figure 17. Simulation of a transmitter implanted in the right forearm. The user has interactively drawn a line segment in the $3 D$ virtual scene. A graph showing signal strength along that line is displayed.

RF propagation to and from medical implants. For example, we have implemented interactive tools for probing the 3D data fields. One tool enables the researcher to move the motion-tracked wand through the virtual scene, yielding a continuously updated display of the value of the data field at the position of the 3D wand. Another tool enables the user to interactively stretch a line segment through virtual body, and to generate graphs of the 3D data fields along that path (see Figure 17). We have applied these tools to the results of RF simulations with antennas of various characteristics at various locations throughout the body.

Using the insight gained from interacting with the results of these simulations in the immersive environment, together with additional analysis, we have constructed a statistical model of signal strength path loss as a function of distance. These results are described in reference [1], which received the Best Paper Award at the IEEE 2009 Personal, Indoor, and Mobile Radio Communications Symposium. Further results were presented in an invited paper [2] at the $2009 \mathrm{In}$ ternational Symposium on Applied Sciences in Biomedical and Communication Technologies. Additionally, implant channel models that have been obtained as a result of our work have been adopted by the IEEE 802.15.6 Task Group on BAN that is developing an international communication standard for implantable and wearable devices $[3,4,5]$.

We plan to continue this work in several areas. We will be using our simulation and visualization system to compare antennas of various placements and designs. We also plan to compare our simulation results with experimental measurements and to use our immersive visualization system to investigate deviations of simulations from experiments. In addition, the $3 \mathrm{D}$ visualization platform is an ideal environment to observe and study creeping wave propagation on the body surface. We believe that the immersive visualization environment provides a flexible platform to visualize and understand RF propagation for BANs.

\section{References}

[1] K. Sayrafian-Pour, W. Yang, J. Hagedorn, J. Terrill, K. Y. Yazdandoost, A Statistical Path Loss Model for Medical Implant Communication Channels, Proceedings of the IEEE PIMRC 2009.

[2] W. Yang, K. Sayrafian-Pour, J. Hagedorn, J. Terrill, Simulation Study of Body Surface RF Propagation for UWB Wearable Medical Sensors, IEEE International Symposium on Applied Sciences in Biomedical and Communication Technologies, November 2009.

[3] J. Hagedorn, J. Terrill, W. Yang, K. Sayrafian-Pour, K. Y. Yazdandoost and R. Kohno, A Statistical Path Loss Model for MICS , September 2008, Report to the IEEE P802.15. ID: IEEE 802.15-08-0519-01-0006.

[4] J. Hagedorn, J. Terrill, W. Yang, K. Sayrafian-Pour, K. Y. Yazdandoost and R. Kohno, MICS Channel Characteristic: Preliminary Results, May 2008, Report to the IEEE P802.15. ID: IEEE 802.15-08-0351-00-0006.

[5] K. Y. Yazdandoost and K. Sayrafian-Pour, Channel Model for Body Area Network (BAN), April 2009, Report to the IEEE P802.15. ID: IEEE 802.15-08-0780-020006.

\section{Participants}

John Hagedorn, Judith Terrill, and Adele Peskin. Kamran Sayrafian-Pour and Wen-Bin Yang (NIST ITL).

http://math.nist.gov/mcsd/savg/vis/BodyAreaNetwork 


\section{Mathematical Knowledge Management}

The field of knowledge management deals with the practices used to create, represent, distribute and identify "knowledge." The ubiquity of word processing, the web and the fact that the name of a successful web search engine is commonly treated as a verb reveal the broad impact of these techniques on society.

But these successes are primarily achieved for knowledge that naturally takes a textual form. The characteristics of mathematical knowledge and its publishing present a unique set of challenges. The special symbols and notations require special means to write and represent. The ambiguities created by the disconnect between the familiar notations (mathematical presentation) and the underlying meaning (mathematical semantics) inhibit reuse and accessibility. Both effects create difficulties for finding mathematical material in the creation of search indices as well as in formulating search queries.

As part of the Digital Library of Mathematical Functions project $-a$ web oriented successor to the classic NBS Handbook of Mathematical Functions [1] - we began addressing these issues. In that context we developed a tool for the conversion from TeX, the de facto standard for writing in many scientific fields, to an abstract XML format with MathML - a W3C standard for representing mathematics with growing support in web browsers and applications.

Beyond the flexibility provided for use on the web and accessibility, this format enables further developments for knowledge management. We explore the inference of mathematical semantics from the markup, enhancing the reuse of the formula. Techniques for search within document collections specialized to matching fragments of mathematics have been developed. Additionally, the half-million article corpus of scientific documents at the arXiv is being converted, providing a large set of documents for further use and experimentation.

\section{Bruce Miller}

In the future, interactive computing environments may combine computer algebra, theorem proving, numerical computation, graphics and word processing to create scientific documents containing complete, verified and semantically rich mathematical content. Presumably, such a format would easily support the finding and reuse of such mathematical material for further proof or computation. Meanwhile, most mathematics is at least published, if not created, in a less rigorous, printoriented, fashion. Still, one would like to maximize the accessibility, reuse and finding of such materials.
Moreover, there exists a rich legacy of printed scientific literature that we can hardly afford to abandon.

We will focus here on TeX, a powerful, but quirky, document formatting language, and the de facto standard for authoring in most mathematically oriented scientific fields, due to its support of complex mathematics and high-quality typesetting. It was the clear choice for authoring in Digital Library of Mathematical Functions ${ }^{2}$ project and is the preferred format for the preprint archive at arXiv ${ }^{3}$.

While conversion of TeX to simple HTML was possible, conversion to a more structured XML, particularly supporting the MathML standard was not. We thus developed LaTeXML ${ }^{4}$, a tool for converting LaTeX to XML. The XML format used here preserves the document structure, but is easily transformed to HTML for use on the web. The mathematical content within the document receives special treatment; it is parsed with the goal of at least recognizing the mathematical structure of the expressions. With author supplied declarations more of the semantic intent of the formula may also be inferred. Further work in this area will allow more semantics to be synthesized with less author input, although given the inherent ambiguities of mathematical notation, perfect enhancement cannot be expected. In any case, the better the semantics obtained, the better the reuse in other contexts such as copying to computer algebra systems. Enhanced semantics also enhances search capabilities.

The method used to enable searching for mathematics leverages the advanced state of text search engines. The idea is to convert the mathematics to a textual form that parallels the MathML markup but contains both the mathematical symbols and keywords reflecting the mathematical structure [2]. Search queries are similarly transformed into this textual form. Search accuracy and expressiveness is improved by colocating alternative forms for symbols including aliases, semantic terms and any presentational variations. Since mathematics expressions often contain complicated sub-expressions that are nevertheless not critical to a given search, a technique called query relaxation can be used to allow the match to skip over subexpressions in the quest for a reasonable number of matching documents. By textualizing in parallel to the MathML markup, the matching terms within the search result can be marked for highlighting providing for useful feedback. Figure 18 shows these several search features.

\footnotetext{
${ }^{2}$ http://dlmf.nist.gov/

${ }^{3} \mathrm{http} / / /$ arXiv.org/

${ }^{4}$ http://dlmf.nist.gov/LaTeXML/
} 


\section{Hankel Ai}

Hankel $\sim \mathrm{Ai}$

- 4 matching pages

Search Advanced Help

Your search matched, but the results seem poor.

See Search Help for suggestions.

(0.034 seconds)

\section{§ 4 matching pages}

\section{1: 10.19. Asymptotic Expansions for Large Order}

...

$-10.19 .9$

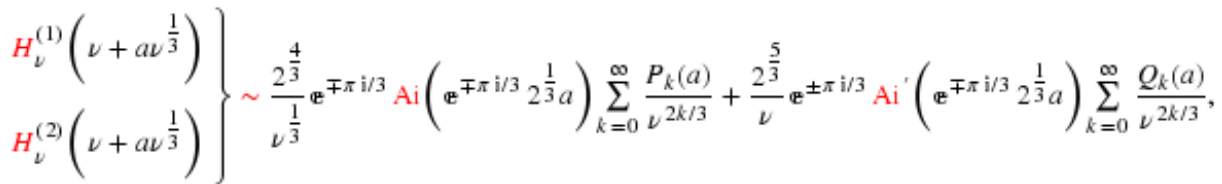

10.19 .13

$$
\left.\begin{array}{l}
H_{\nu}^{(1)^{\prime}}\left(\nu+a \nu^{\frac{1}{3}}\right) \\
H_{\nu}^{(2)^{\prime}}\left(\nu+a \nu^{\frac{1}{3}}\right)
\end{array}\right\} \sim-\frac{2^{\frac{5}{3}}}{\nu^{\frac{2}{3}}} \mathbb{e}^{ \pm \pi \mathrm{i} / 3} \mathrm{Ai}\left(\mathrm{e}^{\mp \pi \mathrm{i} / 3} 2^{\frac{1}{3}} a\right) \sum_{k=0}^{\infty} \frac{R_{k}(a)}{\nu^{2 k / 3}}+\frac{2^{\frac{4}{3}}}{\nu^{\frac{4}{3}}} \mathbb{e}^{\mp \pi \mathrm{i} / 3} \mathrm{Ai}\left(\mathrm{e}^{\mp \pi \mathrm{i} / 3} 2^{\frac{1}{3}} a\right) \sum_{k=0}^{\infty} \frac{S_{k}(a)}{\nu^{2 k / 3}},
$$

Figure 18. A search with a query with both semantic ("Hankel") and presentational ("Ai") terms; the " " stands for asymptotically equal. The result shows how the query was relaxed to skip over function arguments and leading factors to find the best matching asymptotic equation. The term-by-term highlighting gives feedback on how the match was made.

Meanwhile, a project at Jacobs University in Bremen has been carrying out its own research in mathematically aware search, as well as enhancement of semantics. A large body of mathematically oriented documents was needed for experimentation. Thus, we set about to convert the papers in the preprint archive at arXiv from TeX to XML [3]. A cluster of workstations carries out document conversions in parallel.

Currently, 400,000 documents have been processed with the conversion of $60 \%$ being of good quality. This corpus is being used for several experiments in document understanding which attempts to integrate the grammar based parsing of the mathematics with natural language processing of the textual content; clearly both must be understood to fully understand a document.

This exercising of LaTeXML has driven improved coverage and robustness, leading to a more generally useful tool. The rich variety of mathematical notations encountered is key to improving the mathematical grammar for semantic enhancement.

\section{References}

[1] M. Abramowitz and I. Stegun, Eds., Handbook of Mathematical Functions, National Bureau of Standards, 1964.

[2] B. Miller and A. Youssef, Technical Aspects of the Digital Library of Mathematical Functions, Annals of Mathematics and Artificial Intelligence 38 (2003), pp. 121-136.

[3] H. Stammerjohanns, M. Kohlhase, D. Ginev, C. David, and B. Miller, Transforming Large Collections of Scientific Publications to XML, Mathematics in Computer Science, Special Issue on Authoring, Digitalization and Management of Mathematical Knowledge, to appear.

\section{Participants}

Bruce Miller. Abdou Youssef (MCSD and George Washington University). Michael Kohlhase andHeinrich Stamerjohanns (Jacobs University, Bremen, Germany). 


\section{Part III}

\section{Project Summaries}

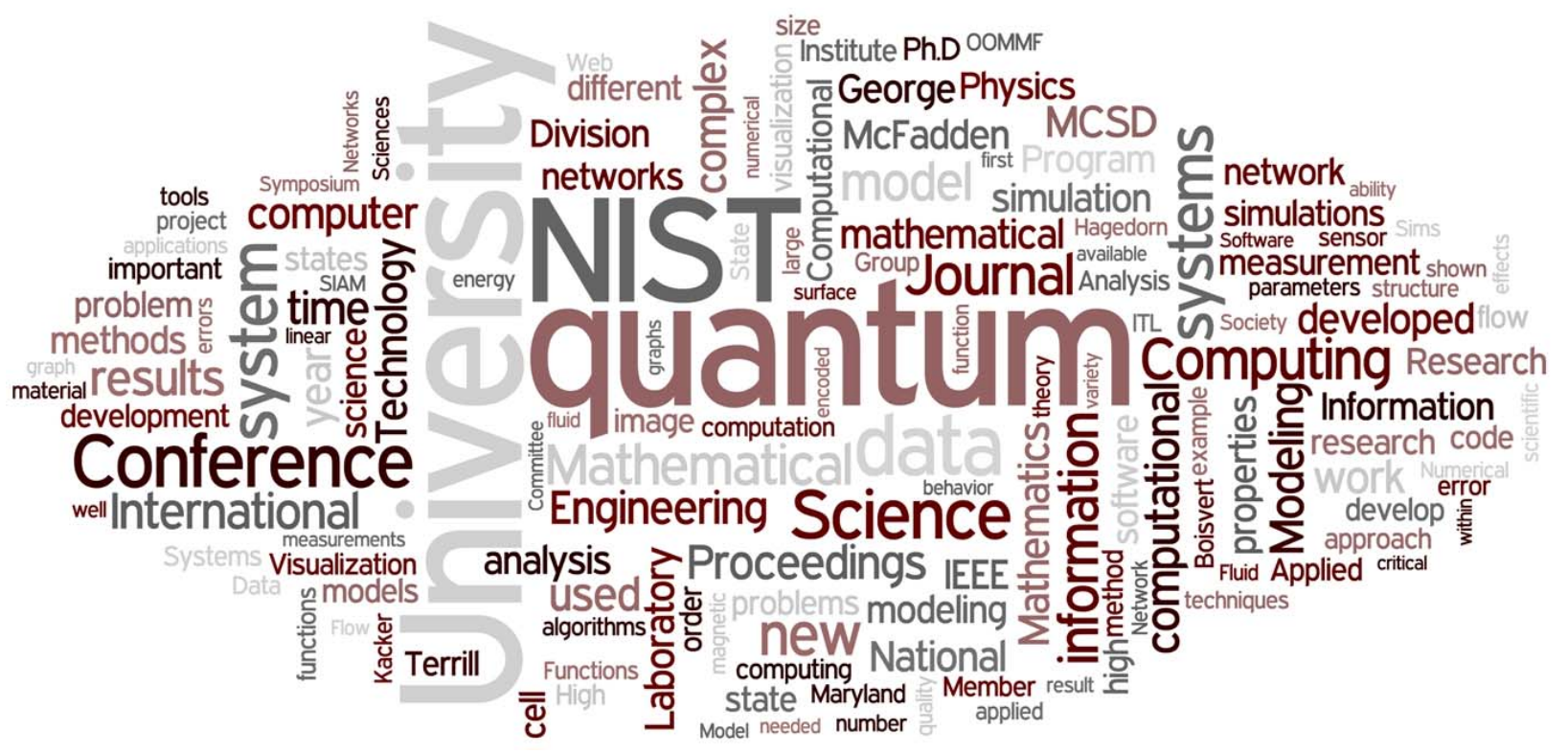




\title{
Information Technology Laboratory Programs
}

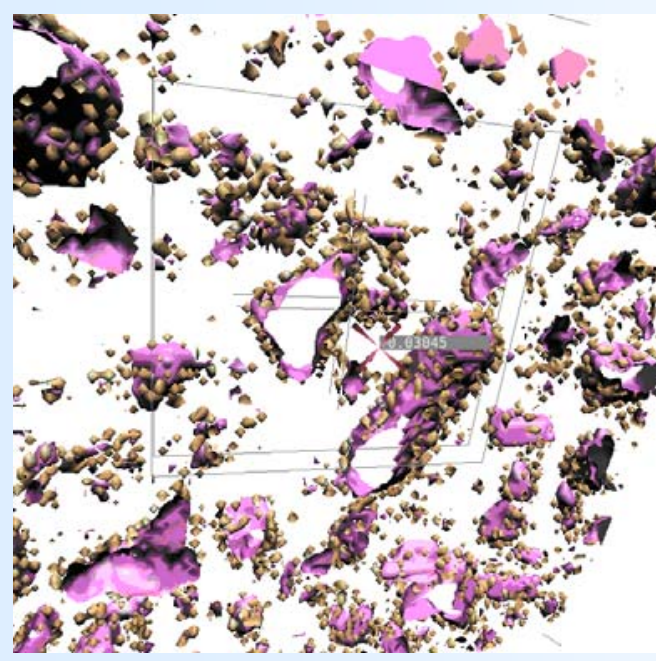

\author{
Enabling \\ Scientific \\ Discovery
}

Information

Discovery, Use and Sharing

Complex Systems

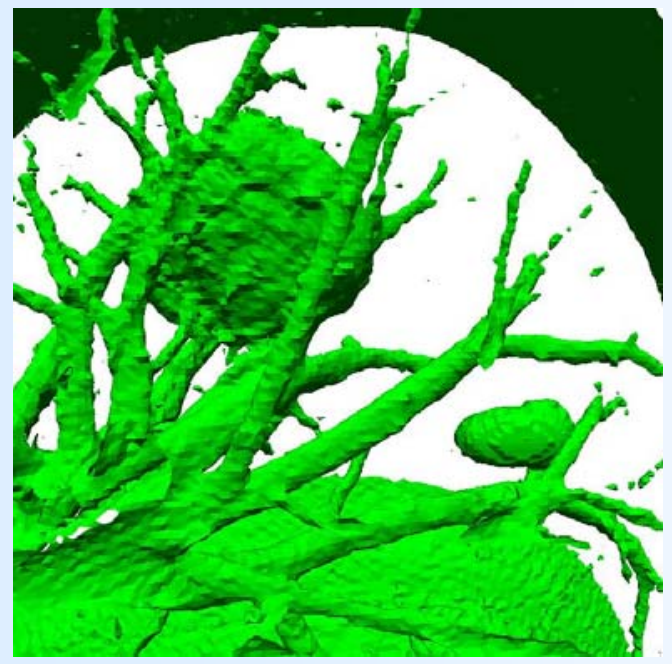

Quantum Information

Virtual Measurement Systems

Pervasive Information Technology

Trustworthy Information Systems 


\section{Enabling Scientific Discovery}

Modern scientific research has become more and more dependent on mathematical, statistical, and computational tools for enabling discovery. The Enabling Scientific Discovery Program promotes the use of these tools to dramatically advance our ability to predict the behavior of a broad range of complex scientific and engineering systems and enhance our ability to explore fundamental scientific processes. This Program focuses on interdisciplinary scientific projects that involve novel computational statistics and the development of simulation methods and software. These efforts will have a foundational impact on scientific discovery throughout U.S. industry, government, and academia. - Anthony Kearsley, Program Manager

\section{Recovering from Physically Impossi- ble Reconstructions in Variational Blind Deconvolution}

Alfred Carasso

See feature article, page 29.

\section{Computational Modeling of the Flow of Dense Suspensions}

\author{
William George \\ Steve Satterfield \\ John Hagedorn \\ Marc Olano \\ Judith Terrill \\ Nicos Martys (NIST BFRL) \\ Virtual Cement and Concrete Testing Lab Consortium
}

See feature article, page 31.

\section{Sparse Representations in High Di- mensional Geometry}

\author{
Bradley Alpert \\ Yu Chen (New York University)
}

Laboratory and clinical diagnostic systems often produce images that lack quantitative information or comparability across imaging systems, which can lead to difficulties in diagnosis and long-term management of disease that requires patient monitoring. Elaborate measurements are taken, digitally processed, then reported in essentially qualitative form. For MRI, functional MRI, and some other processes, formation of the images relies on filtering and Fourier transformation.

Although the computational recovery of functions from their Fourier representations is considered rou- tine, aside from possible efficiency concerns arising from non-grid data, standard approaches assume that the functions are smooth and can be recovered with the discrete Fourier transform (computed via the FFT). When this assumption fails, as is evident from Fourier data that are not small at the highest frequencies measured, some sort of attenuation, or belling, scheme is typically used. This procedure tends to blur sharp features and, being more art than science, can lead to different functions (images) in different systems. Although this problem, and the Gibbs phenomenon, have received considerable attention from mathematicians in recent years, recently developed methods tend to work well only in an asymptotic sense; they do not exploit available data efficiently.

Despite inherent limits on resolution that can be obtained from truncated Fourier data, a change of assumptions from smooth to piecewise smooth can lead to significantly improved recoveries. Procedures implementing this idea are not completely established even for functions (signals) in one dimension; they are yet more challenging in two and three dimensions, where discontinuities may be expected to occur along mostly smooth curves or surfaces. This constraint, which magnifies the advantage of the piecewise smoothness assumption, must be appropriately reflected in the methods used. Bradley Alpert and $\mathrm{Yu}$ Chen are conducting extensive numerical experiments to understand this environment and to develop reliable procedures for these problems.

In recent months, a technique was developed for exact recovery of piecewise polynomial functions, in which the discontinuities between pieces are located by examining the empirical translation matrix of the Fourier data, which has eigenvalues of multiplicity corresponding to the order of the discontinuities. This structure, which is not invariant in the presence of noise or removal of the piecewise polynomial assumption, nevertheless is suggestive of the structure when these stipulations are removed. The eigenvalues then split into clusters, in which the mean of each cluster approaches the corresponding discontinuity with high order convergence. Current numerical experiments and implementations are aimed at turning these observations into a robust method for locating discontinuities. 
Once the discontinuity locations are known, recovery of the discontinuous function is a generally wellconditioned procedure consisting of solving a linear system of equations.

A related, yet more general, challenge is parsimonious, or sparse, representation and recovery of functions under assumptions appropriate to an application. Although there is considerable current interest in the mathematical community in these problems $\left(\mathrm{L}^{1}\right.$-norm minimization, compressive sensing, sparse representation in high-dimensional geometry), and much recent progress (initiated in pivotal work by Candes, Romberg, and Tao) most of the methods being explored are limited to linear spaces. While linearity is a natural starting point, strong evidence suggests that image recovery cannot be done this way yet it nevertheless is within reach. It is the goal of this project to develop procedures for robust recovery of piecewise smooth, or otherwise constrained, functions from Fourier data.

\section{Analysis of Diffusion and Stress in Binary Alloys}

\section{Geoffrey B. McFadden \\ William Boettinger (NIST MSEL)}

The Kirkendall effect refers to the motion of lattice planes in a crystalline binary alloy that is caused by a difference in the rates of diffusion of the two species. The difference in diffusion rates leads to a stress-free stain in the sample, and the process is an example of the coupling between diffusion and elasticity during phase transformations. Current interest in stress generation in thin films due to diffusion processes has stimulated new interest in this classical problem. The effect can lead to measurable shape changes perpendicular to the direction of diffusion [1]. It is often detected by a shift of inert markers parallel to the diffusion direction; that is, deformation with pure axial displacement $[2,3]$.

In current work we are analyzing the timedependent bending of a bimetallic strip due to interdiffusion in a binary alloy. In this case the deformation is due to diffusion across the short dimension of the strip, so that the deformation and diffusion are colinear. We have considered two cases: first, diffusion in a single-phase material, in which the results, which employ a Fourier method, couple simple beam theory and diffusion. The second case considers a two-phase material, in which the interface separating the two phases moves in response to the concentration gradients induced in the sample. In that case we employ a similartity solution for short times, when end effects of the sample are negligible, followed by a numerical solution using finite differences for the long time solu-

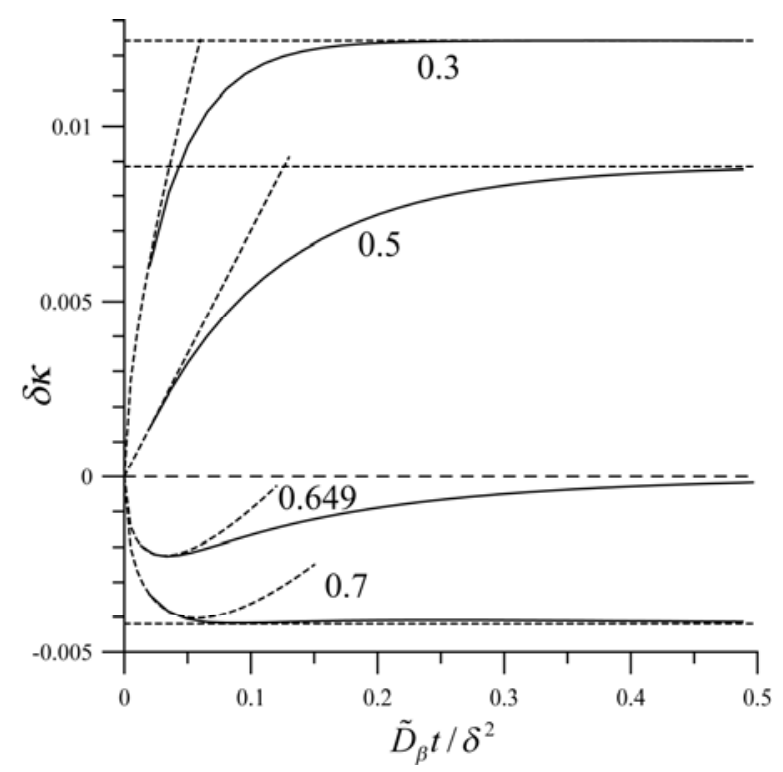

Figure 19. The solid curves show the dimensionless curvature $\delta \kappa$ of the beam vs. the dimensionless time $t D_{\beta} \delta^{2}$ for various initial positions of the interphase boundary $z / \delta=0.3,0.5,0.649,0.7$, where $\delta$, where is the beam thickness and $D_{\beta}$ is the diffusion coefficient. The dashed lines are the initial approximation and the long-time limit.

tion representing the approach to steady-state behavior (see Figure 19). The results are in good agreement with previously reported experiments [4].

[1] W. J. Boettinger, G. B. McFadden, S. R. Coriell, R. F. Sekerka, and J. A. Warren, A Model for the Lateral Deformation of Diffusion Couples, Acta Materialia 53 (2005), pp. 1995-2008.

[2] W. J. Boettinger, J. E. Guyer, C. E. Campbell, and G. B. McFadden, "Computation of the Kirkendall Velocity and Displacement Fields in a One-dimensional Binary Diffusion Couple with a Moving Interface," Proceedings of the Royal Society A 463 (2007), pp. 3347-3373.

[3] J. A. Dantzig, W. J. Boettinger, J. A. Warren, G. B. McFadden, S. R. Coriell, and R. F. Sekerka, "Numerical Modeling of Diffusion-induced Deformation," Metallurgical and Materials Transactions 37A (2006), pp. 2701-2714.

[4] W. J. Boettinger and G. B McFadden, "Bending of a Bimetallic Beam Due to the Kirkendall Effect," Journal of Phase Equilibrium and Diffusion, in press (2010). 


\section{Modeling of Self-Similar Grain Size Distributions}

\author{
Geoffrey. B. McFadden \\ C.S. Pande (Naval Research Laboratory)
}

Polycrystalline solids typically consist of a large number of grains that differ in the orientation of their crystallographic axes. These grains have a distribution of sizes, and are separated by curved grain boundaries that in turn meet at grain boundary junctions. Driven by combinations of elasticity, diffusion, or capillary forces the grain boundaries migrate in time, and the distribution of grain sizes consequently evolves dynamically. A statistical description of the process is given by a grain size distribution function, which is assumed to depend on a mean grain radius and time. Grain growth under usual circumstances is known to approach a quasi-stationary distribution of grain sizes after a transient period. The quasi-stationary state in a wide variety of materials exhibits a scaling property, such that the grain size distribution has an invariant form when expressed in terms of the grain size scaled by its mean value. An accurate description of the spatial and temporal evolution of a polycrystal from an initial stage, through the transient period, and finally to the quasi-stationary state is still only poorly understood. In this work, a size-based continuum stochastic formulation is presented based on topological considerations. This analysis leads to a Fokker-Planck equation for the size distribution which admits a similarity solution that describes the long time behavior of the grain growth. The solution for the case of twodimensional grain growth recently appeared [1]. The three-dimensional version of this problem is has been successfully addressed this year [2]. The resulting grain size distributions are in good agreement with those obtained from computer simulations (see Figure 20),

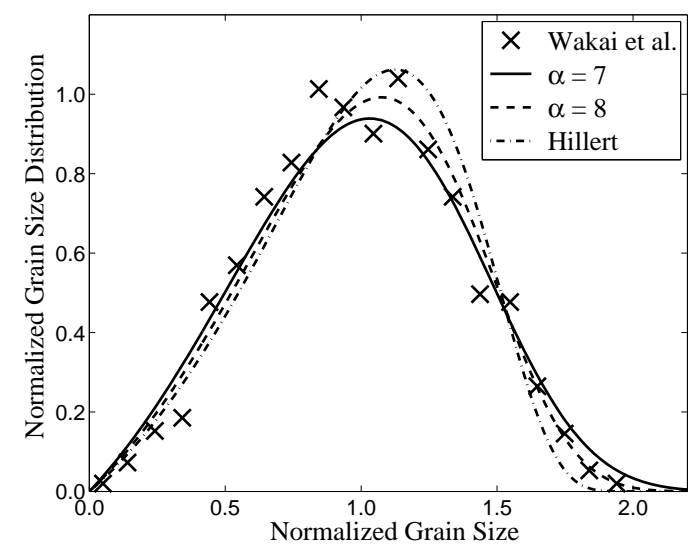

Figure 20. Comparison of numerical simulation results (data points) with three self-similar grain size distributions from Ref. [2]. indicating the validity of the stochastic approach.

[1] C. S. Pande, K. P. Cooper, and G. B. McFadden, "Grain Size Distribution in Two Dimensions in the Long Time Limit," Acta Materialia 56 (2008), pp. 5304-5311.

[2] C. S. Pande and G. B. McFadden, "Self-Similar Grain Size Distribution in Three Dimensions," Acta Materialia, in press.

\section{Modeling Fluid Flow in Materials Processing}

Geoffrey B. McFadden

$P$. Aaron Lott

Sam R. Coriell (NIST MSEL)

Daniel Anderson (George Mason University)

Bruce Murray (SUNY Binghampton)

The study of the stability of a fluid-fluid interface is important in a number of scientific and technological applications. In this project we consider two fluid layers separated by a horizontal planar interface subject to a vertical temperature gradient. The effects of various driving forces on the stability of the system can be taken into account, including buoyancy (known as Rayleigh-Benard convection), the effects of bulk density differences (known as Rayleigh-Taylor instabilities), and the effects of surface tension gradients along the interface (known as Marangoni instabilities). If the two layers represent different phases of the same material the stability results for a two-phase bi-layer system are quantitatively and even qualitatively different than for those of an immiscible system. To estimate the relative importance of these types of instabilities for a two-phase system, we have previously considered a bi-layer geometry in which a horizontal fluid-fluid interface separates two semiinfinite layers of a single component fluid [1,2]. Recently we studied [3] a novel version of the two-phase system in which the onset of the instability is oscillatory in time. This instability was first noted by researchers at the University of Minnesota, although the physical origins of the instability are poorly understood. We were able obtain both numerical and analytical approximations which help explain the physical mechanism of the instability. This year we have worked on an extension of the two-phase, bi-layer problem to the case of a binary alloy system [4]. This work is relevant to experimental studies of "monotectic" systems (in our case the aluminum-indium system) in which a liquid parent phase undergoes a phase transition leading to two daughter phases, one a liquid and one a solid. The geometry of the two-phase system is often composed of rod-like inclusions of one phase within the other, forming a composite that has useful technological applications. An open problem is to pre- 
dict the inter-rod spacing in such systems, which is found to be sensitive to the flow conditions at the liquid-liquid phase boundary. In another related study, the effect of buoyant convection on the stability of a mushy zone in a ternary alloy has been considered [5]. The mushy zone is an idealized model of a two-phase region where liquid and solid co-mingle. References [4] and [5] are invited papers for a special edition of the Journal of Fluid Mechanics celebrating the seventieth birthday of its editor, S. H. Davis, who has served as the Ph.D. advisor for several MCSD postdocs. G. McFadden is acting as a guest co-editor for this volume.

[1] G. B. McFadden, S. R. Coriell, K. F. Gurski and D. L. Cotrell, "Onset of Convection in Two Liquid Layers with Phase Change," Physics of Fluids 19 (2007), 104109.

[2] G. B. McFadden, S. R. Coriell, K. F. Gurski and D. L. Cotrell, "Convective Instabilities in Two Liquid Layers, ” Journal of Research of NIST 112 (2007), pp. 271281.

[3] G. B. McFadden and S. R. Coriell, "Onset of Oscillatory Convection in Two Liquid Layers with Phase Change," Physics of Fluids 21 (2009), 034101.

[4] G.B. McFadden, S.R. Coriell, and P.A. Lott, "Onset of Convection in Two Layers of a Binary Liquid," Journal of Fluid Mechanics, in press (2010).

[5] D. M. Anderson, G. B. McFadden, S. R. Coriell, and B. T. Murray, "Convective Instabilities During the Solidification of an Ideal Ternary Alloy in a Mushy Layer," Journal of Fluid Mechanics, in press (2010).

\section{Materials Data and Metrology for Applications to Machining Processes, Frangible Ammunition, and Body \\ Armor}

\section{Timothy Burns}

Steven Mates (NIST MSEL)

Richard Rhorer (NIST MEL)

Eric Whitenton (NIST MEL)

Debasis Basak (Orbital Sciences Corporation)

High-speed metal machining processes can cause extremely rapid heating of the workpiece material. If this material is a carbon steel, a small region of the workpiece is deformed plastically at a strain rate on the order of $10,000 \mathrm{~s}^{-1}$, on a time interval on the order of one millisecond. On this time interval, the material undergoes a change in temperature from ambient $(\sim 25$ ${ }^{\circ} \mathrm{C}$ ) to a temperature on the order of magnitude of 1000 ${ }^{\circ} \mathrm{C}$, as a result of dissipation due to rapid shear in the primary and secondary shear zones. This means that, in a fairly routine cutting operation on a modern machin-

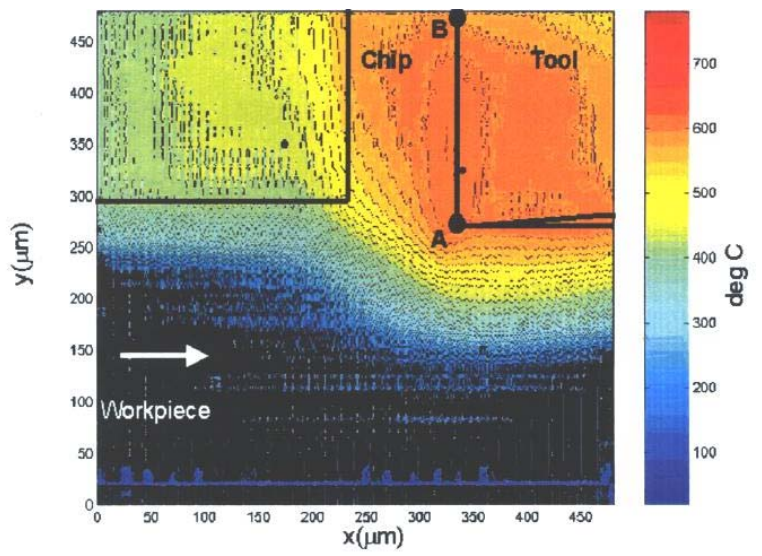

Figure 21. Thermal image of high-speed orthogonal machining of AISI 1045 steel; temperature is a maximum along the chip-tool interface $(A-B)$ due to friction.

ing center, a heating rate on the order of one million degrees centigrade per second is not uncommon for iron-carbon alloys of interest in manufacturing; see Figure 21 [1]. This poses a challenge for modeling the constitutive response, i.e., the material flow stress vs. the strain, strain rate, temperature, etc., of such materials, for use in computer simulations of machining operations.

As part of a research program on the measurement and modeling of machining operations, we have developed the NIST Pulse-Heated Kolsky Bar Laboratory for the study of material response at high temperatures and rates of loading [2]. The Laboratory is a unique testing facility in which the strength of metals can be measured under conditions of rapid DC resistive heating, followed by rapid compression. The

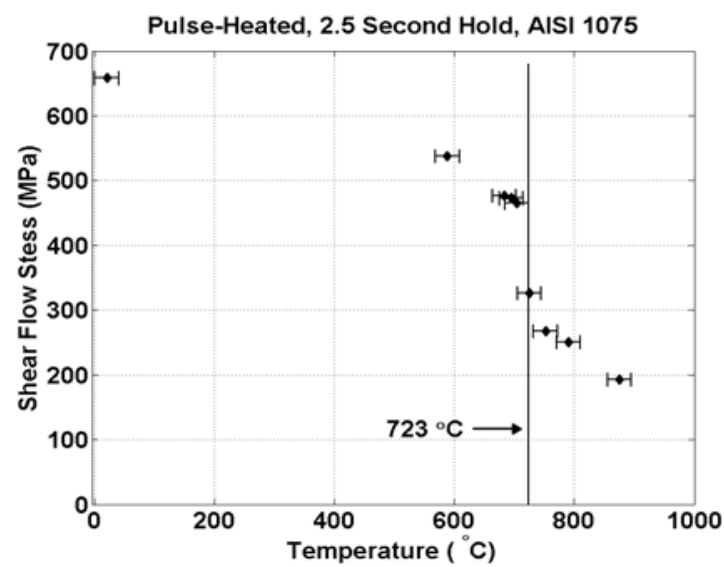

Figure 22. Pulse-heated Kolsky bar data on shear flow stress vs. temperature, at a true compressive strain of 0.1 , and a true strain rate of $3500 \mathrm{~s}^{-1}$ [3]; sample microstructures were a uniform fine pearlite prior to testing; error bars denote 2o [2]; upper left data point corresponds to room-temperature test in which sample was not pre-heated. 
flow stress can be measured in metals that have been pre-heated to uniform temperatures of up to $1200^{\circ} \mathrm{C}$, at heating rates up to $6000{ }^{\circ} \mathrm{C} \mathrm{s}^{-1}$, and then dynamically loaded at strain rates up to the order of $10,000 \mathrm{~s}^{-1}$. The facility is also being used for more conventional roomtemperature testing of materials, such as frangible ammunition made from sintered powders, in support of the NIST Office of Law Enforcement Standards (OLES).

In recent work [3], new pulse-heated compression test results on AISI 1075 steel were presented, in which a rapid phase transformation from pearlite to austenite was observed to take place near the austenization temperature $\left(723{ }^{\circ} \mathrm{C}\right)$ of the material. We are currently studying the implications of this behavior for finiteelement simulations of high-speed machining of this material.

[1] M. A. Davies, H. Yoon, T. L. Schmitz, T. J. Burns, and M. D. Kennedy, "Calibrated Thermal Microscopy of the Tool-Chip Interface in Machining," Journal of Machining Science and Technology 7(2) (2003), pp. 166190.

[2] S. P. Mates, R. L. Rhorer, E. P. Whitenton, T. J. Burns, and D. Basak, "A Pulse-heated Kolsky Bar Technique for Measuring Flow Stress of Metals Subjected to High Loading and Heating Rates," Experimental Mechanics 48 (2008), pp. 799-807.

[3] T. J. Burns, S. P. Mates, R. L. Rhorer, E. P. Whitenton, and D. Basak, "Dynamic Properties for Modeling and Simulation of Machining: an Update of Results from the NIST Pulse-heated Kolsky Bar," in 12th CIRP Conference on Modeling of Machining Operations, 2009. San Sebastian, Spain.

\section{Boundary Slip Effects on the Stabil- ity of Spiral Poiseuille Flow}

\section{P. Aaron Lott}

Geoffrey B. McFadden

David L. Cotrell (Lawrence Livermore National Lab)

Advances in micro-fabrication techniques have provided significant understanding into the behavior of fluids at small scales. Small scale experiments, with flow driven by pressure gradients, drainage, shear, or an electric field have reported a breakdown of the noslip boundary condition, with observed slip lengths as large as microns. In addition, numerical studies of fluids are often performed with no-slip boundary conditions, and the effects of introducing slip as a model of surface roughness is of current interest.

We consider the effect boundary slip has on the stability of the spiral Poiseuille flow, which is an annular flow with an applied pressure gradient [1]. We have found that under certain conditions, slip has a moderate effect on the linear stability of the flow. Numerical results indicate that linear stability is sensitive to the ratio between the inner and outer cylinder radii, as well as the slip length. Changes in these parameters cause the flow to be stabilized or destabilized in reference to a flow where no-slip conditions are applied; this change in stability is indicated by change in critical Taylor number. The dependence of stability on radius ratio becomes more complicated as the Reynolds number is increased; in particular, the flow becomes destabilized by small ratios, stabilized by mid-range ratios and de-stabilized again for large ratios. We have computed estimates of the critical radius ratios where slip has no effect on the stability of the flow. We are currently examining whether changes in the angular velocity of the inner and outer cylinders exhibits similar variations in stability of the flow.

[1] D.L. Cotrell, P.A. Lott and G.B. McFadden, "Boundary Slip Effects on the Stability of Spiral Poiseuille Flow," in preparation.

\section{Nanostructures, Nanooptics, and How to Squeeze the Light out of Quantum Dots}

\author{
James S. Sims \\ John Kelso \\ Garnett W. Bryant (NIST PL)
}

Research and development of nanotechnology, with applications ranging from solar cells and light emitting diodes to smart materials to quantum computation to biolabs on a chip, is of high national priority. Semiconductor nanoparticles, also known as nanocrystals and quantum dots, are one of the most intensely studied nanotechnology paradigms. Nanoparticles are typically $1 \mathrm{~nm}$ to $10 \mathrm{~nm}$ in size, with $10^{3}$ to $10^{6}$ atoms. Precise control of particle size, shape, and composition allows one to tailor charge distributions and control quantum effects to tailor properties completely different from the bulk or from small clusters. As a result of enhanced quantum confinement effects, nanoparticles act as artificial man-made atoms with discrete electronic spectra that can be exploited as light absorbers for solar cells, light sources for novel enhanced lasers and light emitting diodes, discrete components in nanoelectronics, qubits for quantum information processing, and enhanced ultrastable fluorescent labels for biosensors to detect, for example, cancers, malaria or other pathogens, and to do cell biology. 
We are working with the NIST Physics Lab to develop computationally efficient large scale simulations of such nanostructures. We are also working to develop real-time (immersive and desktop) visualization techniques and tools to enable analysis of highly complex computational results of this type. In particular, we are studying the electrical and optical properties of semiconductor nanocrystals and quantum dots. In the most complex structures this entails modeling structures with on the order of a million atoms. Highly parallel computational and visualization platforms are critical for obtaining the computational speeds required for a systematic, comprehensive study.

While it often is easy to define the simple subsystems that make up a complex, heterogeneous nanosystem [1], it may be difficult to explicitly define an entire large-scale structure. A novel feature of our code is the ability to link together heterogeneous nanosubsystems. Since we can do multiprocessor runs routinely we have the basic building blocks for making larger structures by "stitching" together disparate subsystems into composite structures, each separate component subsystem being a smaller multiprocessor run. We are currently focusing on modulating and controlling the optical properties of self-assembled quantum dots using external strain [2-5].

To understand the coupling between externally imposed strains and the electronic states of the selfassembled quantum dots, we have developed an atomistic tight-binding theory of the confined states in quantum dots that incorporates the local strain due to atomistic lattice mismatch and the externally imposed strain due to surface acoustic waves to applied stressors or to the bend in a vibrating nanomechanical oscillator. Both local strain and the externally imposed strain are important, so we include them on an equal footing via an atomistic valence force field approach, with the externally imposed strain modeled as a distortion of the lattice on the boundary of structure. A full tightbinding model including an sp3s* orbital model and spin-orbit effects is used [2-5]. To understand how applied stress can be used to actively control dot opti- cal properties, dots buried at points with different strain in a nanobridge oscillator or cantilever are considered. The dependence of the quantum dot electronic states and optical transitions on the coupling to bending modes of the oscillator is studied. Both isolated and coupled dots are considered. Applied strain can be used to transfer electrons and holes between vertically or laterally coupled dots. Level crossings for electron and hole states are found. State symmetries can be significantly distorted by the applied stress. Transitions can be strongly polarized parallel or perpendicular to the external strain or suppressed, depending on how the dots are stressed. The dependence on applied stress can be seen as a competition between the internal and external stress that can either enhance or suppress the local strain at the dot, depending on how the external stress is applied.

We have found that the applied strain can make large changes in the exchange splitting between bound electron-hole pairs (excitons) optically bright states to modify the exciton fine structure induced by QD asymmetry or atomistic effects, can induce crossing between the bright states for certain strains, and can be used to rotate the polarized response of the bright states. These capabilities should be useful for applications such as entangled photon sources, where the elimination of asymmetric exchange splitting is essential, or cavity-coupled QDs, where control of the exchange-induced dark and bright states is needed. Analysis shows that the applied strain tunes the size and phase of the exchange coupling which, in turn controls the phase of the exciton state and the resulting polarization. Such phase control is critical for applications in quantum science.

As the computational model has been extended to handle more complex and larger systems by including not only the nanostructures but also the substrate and environment around them, parallel processing has become even more necessary. We use both shared memory OpenMP parallelization and distributed memory message passing.

To study the increasingly larger systems we are working with, we have also expanded the visualization 
capability with multiple selection tools that can sum up charge density in different types of regions. This year we made improvements in: speed - much faster building, loading and higher graphic frame rate for a given number of orbitals; capacity - a million orbitals can be displayed with an interactive frame rate; and dynamic updates - all displays are dynamically updated as orbitals and selection criteria are modified. Dynamic updates give immediate feedback to the researcher.

New capabilities in the visualization (see Figure 23 for a snapshot of the interface) include:

- Selection of which orbitals to display. Orbitals are currently represented using "fatpoints," i.e., spheres drawn by a GPU shader program. A P orbital is represented by two spheres and an S orbital by one sphere. The spheres are colored and sized as specified by the orbital data.

- Dynamic visual updating of which orbitals lie inside or outside specified geometric objects.

- Tools to dynamically exclude orbitals that are greater or less than a specified size.

- Assistance of a gnomon object to display orbital orientation, the option to display all orbitals at a fixed size, and to magnify all orbitals.

- A dynamically displayed status window showing the number of visible orbitals, and the exact position and orientation of the selection geometry.

- Dynamic summing of visible orbitals, with optional orbital coefficients.

[1] G. W. Bryant and W. Jaskolski, "Electronic Properties of Quantum-dot Molecules," Physica E 17 (2003), pp. 40-41.

[2] G. W. Bryant, N. Malkova, J. Sims, M. Zielinski, W. Jaskolski and J. Aizpurua, "Re-engineering the Optics of Quantum Dots with Mechanical Strain," in Optics of Excitons in Confined Systems (OECS11), Madrid, Spain, September 7-11, 2009.

[3] G. W. Bryant, N. Malkova, J. Sims, M. Zielinski, W. Jaskolski and J. Aizpurua, "Re-engineering the Optics of Quantum Dots with Mechanical Strain," in Proceedings of the Electronic Materials Conference, University Park, PA, June 24-26, 2009.

[4] G. W. Bryant and N. Malkova, "Engineering the Optics of Quantum Dots Using Strain," in Proceedings of the 2009 Nanoelectronic Devices for Defense \& Security (NANO-DDS) Conference, Fort Lauderdale, FL, September 21-25, 2009.

[5] N. Malkova, G. W. Bryant, and J. Sims, "Straining Quantum Dot Excitons," in Proceedings of the Materials Research Society Fall Meeting, Boston, MA, November 30-December 4, 2009.

[6] J. Sims, G. W. Bryant, W. L. George, T. G. Griffin, J. G. Hagedorn, J. T. Kelso, T. M. Olano, A. P. Peskin, S. G. Satterfield, and J. D. Terrill, "Computation and Visualization of Nano-structures and Nano-optics," Computing and Visualization in Science.

\section{Computational Modeling and Visu- alization of Cement Paste Hydration and Microstructure Development}

\author{
William George \\ Steven Satterfield \\ Judith Terrill \\ Jeffrey Bullard (NIST BFRL) \\ Edith Enjolras (NIST BRFL) \\ Clarissa Ferraris (NIST BRFL) \\ Edward Garboczi (NIST BFRL) \\ Nicos S. Martys (NIST BFRL) \\ Paul E. Stutzman (NIST BRFL)
}

When cement powder is mixed with water, the hydration process that transforms the paste from a fluid suspension into a hardened solid involves complex chemical and microstructure changes. Understanding and predicting the rates of these changes is a longstanding but extremely challenging technological goal. Fundamental computational modeling of the hydration of cement is difficult because it involves a large number of coupled nonlinear rate equations that must be solved in an irregular three-dimensional spatial domain.

To address these challenges, we are applying a new computational model called HydratiCA, which has several advantages over previous attempts to model cement paste hydration. HydratiCA is based on the principles of mass action and detailed balances and uses stochastic cellular automaton algorithms to simultaneously model 3-D reaction and transport phenomena. This allows us to track the detailed kinetics and equilibria that occur in a diverse range of cementitious systems. At the length scales required to finely resolve the reaction mechanisms and microstructure changes in cement paste, HydratiCA must take small time steps (approximately $10^{-5}$ per second) to remain numerically stable. In other words, tens of millions of time steps are required to simulate the behavior of cement paste for just one hour. Therefore, parallelization of the model is important so that we can simulate systems that are large enough to be realistic, avoiding finite size effects, and still be able to complete the simulations in a reasonable amount of time. Visualization of the output of this model is important both for validation and to understand the distribution of phases in 3D. The output is a 3D volume of data with percentage values for each of multiple material phases at each lattice site. Over the course of the simulation time, a series of data volumes is produced at the time values of interest.

This year we modified the HydratiCA simulator to use XML encoded parameter files. These changes have simplified the automatic validation of input and has allowed for the easy development of a GUI inter- 


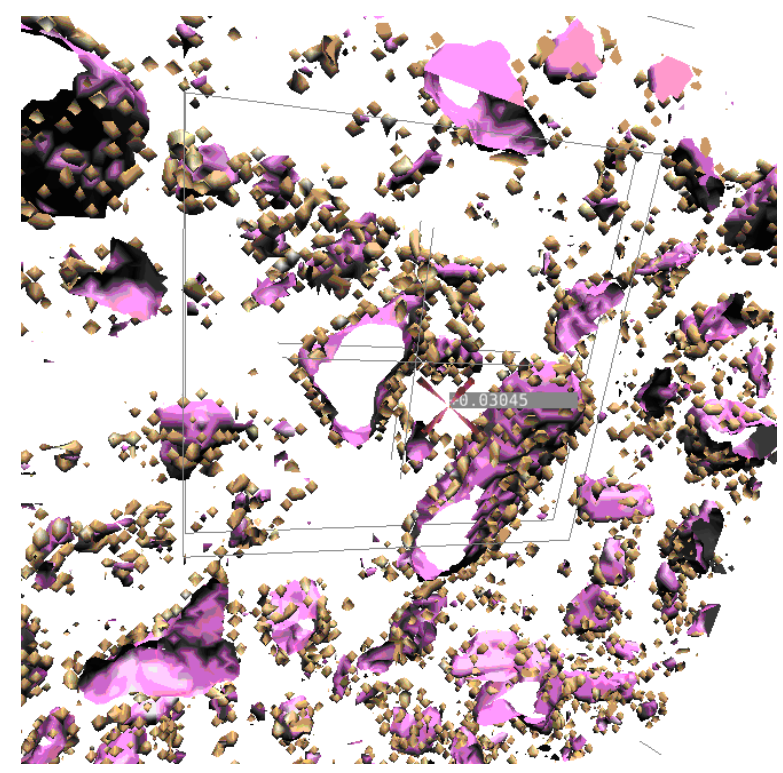

Figure 24. Snapshot from a visualization of formation of slaked lime. The number in the figure is generated with the virtual cement analysis probe and indicates the concentration of the $\mathrm{Ca}^{++}$ion at the point marked by the $3 D$ cross glyph.

face for creating and editing such files. We have improved the checkpoint/restart handling of HydratiCA such that no post-processing or pre-processing of files is needed when restarting a simulation. This greatly simplifies the job of running long simulations within batch systems that enforce relatively short runtimes compared to the runtime of a complete simulation.

We were awarded 540,000 CPU-hours on the NASA Advanced Supercomputing machine Columbia for this project. This time will be used to run HydratiCA for the more compute-intensive simulations required in this project. A sample visualization of our results is shown in Figure 24. We have completed a large number of validation runs and written a paper on the result, which has been accepted for publication. We will be using HydratiCA to help resolve two prominent hypotheses for reduced alite dissolution rates during the induction period.

[1] J. W. Bullard, E. Enjolras, W. L. George, S. G. Satterfield, and J. E. Terrill, "Validating a Scalable Parallel Computer Model of Cement Hydration and Microstructure Development," Modeling and Simulation in Materials Science and Engineering, accepted.

[2] J. W. Bullard, C. F. Ferraris, E. J. Garboczi, N. S. Martys, P. E. Stutzman, and J. E. Terrill, "Virtual Cement and Concrete," Innovations in Portland Cement Manufacturing, in press.

\section{Micromagnetic Modeling}

\author{
Michael Donahue \\ Donald Porter \\ Robert McMichael (NIST CNST) \\ June Lau (NIST MSEL)
}

http://math.nist.gov/oommf/

Advances in magnetic devices such as recording heads, field sensors, magnetic nonvolatile memory (MRAM), and magnetic logic devices are dependent on an understanding of magnetization processes in magnetic materials at the nanometer level. Micromagnetics, a mathematical model used to simulate magnetic behavior, is needed to interpret measurements at this scale. MCSD is working with industrial and academic partners, as well as with colleagues in NIST CNST, MSEL, and EEEL, to improve the state-of-the-art in micromagnetic modeling.

Michael Donahue and Donald Porter have developed a widely used public domain computer code for doing computational micromagnetics, the ObjectOriented Micromagnetic Modeling Framework (OOMMF). OOMMF serves as an open, welldocumented environment in which algorithms can be evaluated on benchmark problems. OOMMF has a modular structure that allows independent developers to contribute extensions that add to the basic functionality of OOMMF. OOMMF also provides a fully functional micromagnetic modeling system, handling both two and three-dimensional problems, with sophisticated extensible input and output mechanisms. In fiscal year 2009 alone, the software was downloaded more than 5,200 times, and use of OOMMF was acknowledged in more than 120 peer-reviewed journal articles. OOMMF has become an invaluable tool in the magnetics research community.

Key developments in OOMMF last year include:

1) Addition of multi-threaded computation in the modules of OOMMF for use on multi-processor, multi-core computers, enabling faster (observed speed-up of four to seven times) and larger simulations, and more efficient utility of common computing platforms [1].

2) Creation of additional modules tailored for the simulation of polycrystalline materials, common in practical device designs [4].

In addition to the continuing development of OOMMF, the project also does collaborative research using OOMMF $[2,3]$. The project plays an instrumental role in the 2007 Innovation in Measurement Science project "Magnetic nanostructures for post-CMOS electronics," with CNST, MSEL and EEEL. We also provide modeling and simulation consultation for work in EEEL on spin momentum transfer. In collaboration 
with the University of Konstanz we have used OOMMF to study magnetic structures in nanowires. The MCSD project produced four journal papers and four conference presentations this past year.

Work in progress includes a continuing effort to develop techniques to minimize or control for errors introduced when the spatial resolution of the computation grid does not exactly represent the boundaries of the simulated material. Further progress on multithreaded computation is focused on designing computations to more effectively use limited memory bandwidth on non-uniform memory access architectures. Another focus area is the use of OOMMF to explore spintronic designs for magnetic memory and logic devices, including a new collaboration with University of Alabama.

[1] M. J. Donahue, "Parallelizing a Micromagnetic Program for Use on Multi-processor Shared Memory Computers," IEEE Transactions on Magnetics 45 (2009), pp. 3923-3925.

[2] W. C. Uhlig, M. J. Donahue, D. T. Pierce and J. Unguris, "Direct Imaging of Current-driven Domain Walls in Ferromagnetic Nanostripes," Journal of Applied Physics 105 (2009), 103902.

[3] M. D. Stiles, W. M. Saslow, M. J. Donahue, and A. Zangwill, "Reply to "Comment on "Adiabatic domain wall motion and Landau-Lifshitz Damping," Physical Review B 78 (Dec. 2008), 216402.

[4] J. W. Lau, R. D. McMichael, and M. J. Donahue, "Implementation of Two-dimensional Polycrystalline Grains in Object Oriented Micromagnetic Framework," Journal of Research of the National Institute of Standards and Technology 114(Jan 2009), pp. 57-67.

\section{OOF: Finite Element Analysis of Material Microstructures}

\author{
Stephen A. Langer \\ Andrew C.E. Reid \\ Valerie R. Coffman \\ Gunay Dogan (Cornell University)
}

http://Www.ctcms.nist.gov/oof/

The OOF Project, a collaboration between MCSD and the NIST Material Science and Engineering Laboratory, is developing software tools for analyzing real material microstructure. The microstructure of a material is the (usually) complex ensemble of polycrystalline grains, second phases, cracks, pores, and other features occurring on length scales large compared to atomic sizes. The goal of OOF is to use data from a micrograph of a real material to predict the macroscopic behavior of the material via finite element analysis. OOF is intended to be a general-purpose tool,

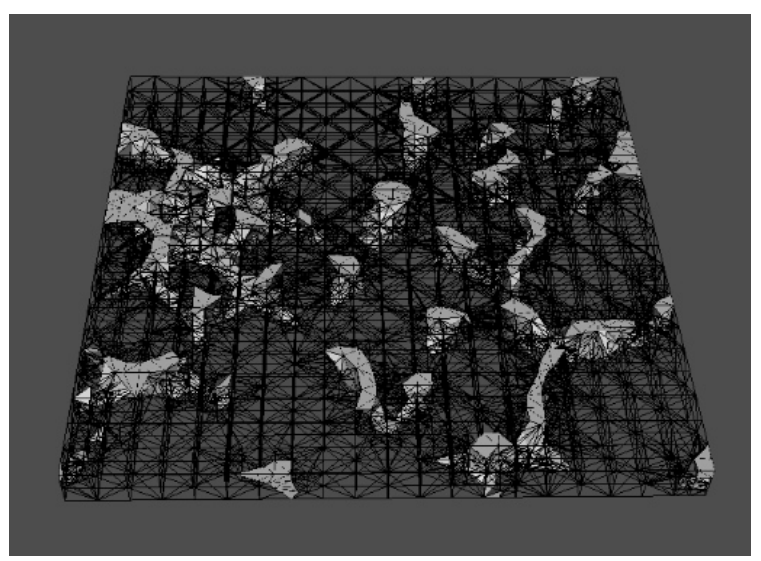

Figure 25. Preliminary data shows that OOF2 can achieve up to a 100 fold reduction in the number of elements required to mesh a $3 D$ micrograph of trabecular bone, compared to the simple approach of creating one element per voxel.

applicable to a wide variety of microstructures in a wide variety of physical situations.

During FY 2009 the OOF team concentrated on improving the non-linear and time dependent solvers in the $2 \mathrm{D}$ program, OOF2, and on developing the $3 \mathrm{D}$ version, OOF3D. Numerous bugs and glitches delayed the release of OOF2 version 2.1, which will be the first version to solve time dependent problems. There were, however, three bug-fix releases of 2.0.5 this past year. Including all versions, OOF2 was downloaded 1,204 times from the OOF website this past year $(10 \%$ more than last year, during which seven minor versions were released). OOF2's predecessor, OOF1, is still available and was downloaded 265 times. This is significantly less than last year, which is good.

As usual, the OOF customer base is broad and difficult to define, since users aren't required to register before using the software. 42 users wrote to the developers for help on installing and running the program this year. OOF2 is still available to be run on-line at Purdue University's nanoHUB site, where it had 259 users in 2,420 interactive sessions this year, up about $50 \%$ from last year.

Externally visible progress on the time-dependent and three dimensional versions has been slow this year. However, almost everything that was not working at the end of last year has now been fixed.

OOF3D can now solve simple 3D diffusion problems from start to finish, beginning with a 3D image and ending with a finite element solution. It can load orientation data from 3D electron backscatter diffraction (EBSD) experiments. More 3D mesh refinement methods have been added. Most of the 3D development effort this year went towards optimization and corner cases in the element homogeneity calculation, which computes the fraction of each mesh element that overlaps each voxel type. This surprisingly subtle 
calculation is fundamental to many OOF computations, so it needs to be fast and robust.

OOF2 gained a module for solving RambergOsgood plasticity, which is a form of nonlinear elasticity that can be used to model the plastic deformation of materials. The nonlinear solvers were restructured to be easier to use and to give users more control over the solution process. The Newton and Picard solvers now share infrastructure.

It is now possible to animate the solutions of timedependent problems. This and other aspects of the time-dependence code required major under-the-hood changes in the way in which OOF2 handles progress bars and manages execution threads.

The OOF developers hope that version 2.1, with time-dependence, and version 3.0, with 3D solutions, will both be made available in FY 2010.

[1] A. C. E. Reid, R. C. Lua, R. E. Garcia, V. R. Coffman, and S. A. Langer, "Modeling Microstructures with OOF2," International Journal of Materials and Product Technology 35 (2009), pp. 361-373.

\section{Fast Solvers for Models of Fluid Flow}

\author{
P. Aaron Lott \\ Geoffrey B. McFadden \\ Sam Coriell (NIST MSEL) \\ Howard Elman (University of Maryland) \\ Katherine Evans (Oak Ridge National Laboratory)
}

Numerical simulation of fluids enables improved prediction and design of a wide range of natural and engineered systems. Fluid systems often involve dynamics that occur on disparate length and time scales due to variations in inertial and viscous forces. In order to numerically simulate these dynamics, advanced mathematical modeling and scalable computational methods are required. The principal goal of this project is to develop fast numerical methods for conducting high resolution fluid flow simulations.

In numerical fluid simulations, the majority of time is spent by linear system solvers that reside within the inner loop of the simulation. Reducing the cost of these linear system solves is a challenging problem in computational mathematics. We are developing preconditioners to accelerate the convergence of iterative linear system solvers for linear systems that arise from spectral element discretizations. Preconditioners are chosen as approximations of the linear system that mimic system properties, but provide an efficient means of applying the inverse operation. We take advantage of underlying structure, multiscale features and parallelism present in the discrete system and use domain decomposition coupled with a fast diagonalization procedure to efficiently apply the preconditioner. These techniques have resulted in significant speedup in simulating steady flow models including convection-diffusion systems [1] and incompressible fluids [2]. We are working to develop improved preconditioning strategies that will enable more challenging flow problems to be solved at higher resolution and on modern supercomputers.

We are also extending the use of our preconditioning strategies to unsteady flow models including thermosolutal convection and the shallow-water equations. Many numerical methods for fluid simulation are based on explicit and semi-implicit time integration schemes. However, in order for these methods to be numerically stable, the maximum time step is required to be bounded by the minimum grid space divided by the maximum flow velocity. This causes time steps to become prohibitively small when simulating fast moving flows at high resolution. As modern computing facilities are increasing the ability to perform higher resolution studies, numerical methods must be developed to provide a scalable means of performing these simulations. Fully implicit methods would allow for significantly larger time steps that are independent of the grid resolution. Similar to steady state fluid simulations, the cost of fully implicit time integration is dependent on the expense of subsidiary linear system solves. By developing efficient preconditioning strategies, we are working to lower the cost of these linear system solves to enable scalable high-resolution flow studies.

[1] P. A. Lott and H. C. Elman, "Fast Iterative Solvers for Convection-diffusion Systems with Spectral Elements," Numerical Methods in Partial Differential Equations, accepted.

[2] P. A. Lott and H. C. Elman, "Fast Iterative Solvers for Incompressible Navier-Stokes Equations with Spectral Elements," in preparation.

\section{Parallel Adaptive Refinement and Multigrid Finite Element Methods}

\author{
William F. Mitchell \\ Marjorie A. McClain \\ Eite Tiesinga (NIST PL) \\ Thomas Hanna (NIST PL)
}

http://math.nist.gov/phaml

Finite element methods using adaptive refinement and multigrid techniques have been shown to be very efficient for solving partial differential equations. Adaptive refinement reduces the number of grid points by concentrating the grid in the areas where the action is, and multigrid methods solve the resulting linear 
systems in an optimal number of operations. Recent research has focused on $h p$-adaptive methods where adaptivity is in both the grid size and the polynomial order of approximation, resulting in exponential rates of convergence. W. Mitchell has been developing a code, PHAML, to apply these methods on parallel computers. The expertise and software developed in this project are useful for many NIST laboratory programs, including material design, semiconductor device simulation, and the quantum physics of matter.

This year the effort was focused on two areas. The first is preparation for an experiment to study the effectiveness of several competing strategies for $h p$-adaptive refinement, which involves implementing these methods in PHAML and defining a set of test problems. The second is the application of PHAML to solve the time-dependent Schrödinger Equation for eigenvalues and eigenstates relevant to optical traps for neutral atoms, in collaboration with the Quantum Processes group of NIST's Atomic Physics Division. Understanding the interactions of adjacent atoms corresponding to qubits of a quantum gate involves computing multiple eigenvalues in the middle of the spectrum, with eigenstates that have sharp gradients, which is a very challenging computation. Major accomplishments for FY 2009 include the following.

- Implementation of the final three $h p$-adaptive strategies in PHAML.

- Completion of preliminary experiments to determine parameters to use in the $h p$-adaptive strategies comparative experiment.

- Completion of the definition and implementation of a set of test problems.
- Improvement of the MPI communication layer of PHAML, to improve parallel performance.

- Extension of the class of problems solved by PHAML to include first order and cross derivative terms, and many other minor improvements. Two releases of the code occurred as the code evolved.

- Design and implementation of a method to solve the time-dependent Schrödinger equation with PHAML.

Future work will continue to enhance PHAML with additional capabilities and robustness, complete the experiment to compare the effectiveness of $h p$-adaptive strategies, add OpenMP directives to PHAML for a hybrid MPI/OpenMP parallel implementation for clusters of multi-core computers, apply PHAML to a timedependent model of two dipolar molecules held in a cylindrically symmetric trap, and perform further physics experiments using the code.

[1] W. F. Mitchell, "The hp-Multigrid Method Applied to hp-Adaptive Refinement of Triangular Grids," Numerical Linear Algebra with Applications, to appear.

[2] W. F. Mitchell and M. A. McClain, "A Survey of hpAdaptive Strategies for Elliptic Partial Differential Equations," Annals of the European Academy of Sciences, invited paper, submitted.

[3] W.F. Mitchell, "hp-Adaptive Finite Elements for the Schrödinger Equation," University of Nevada at Reno, April 2009, invited talk.

[4] W. F. Mitchell, "hp-Adaptive Finite Elements in a Parallel PDE Solver," SIAM Conference on Computational Science and Engineering, March 2009, invited.

[5] W. F. Mitchell, "Application of a Parallel Adaptive Finite Element Code to Confined Interacting Atoms," SIAM Annual Meeting, July 2008. 


\section{Information Discovery, Use and Sharing}

Society is awash in data - our ability to amass data has outpaced our ability to use it. Extracting knowledge, information, and relationships from this data is one of the greatest challenges faced by the scientists in the twenty-first century. The data can be as diverse as biological research data, medical images, automated newswire, speech, or video. The Information Discovery, Use, and Sharing Program fosters innovation throughout the information life cycle by developing the measurement infrastructure to enhance knowledge discovery, information exchange, and information usability. The Program enables novel computational approaches to data collection and analysis to be combined with improved interoperability techniques to effectively extract needed information from the wealth of available data. - Mary Brady, Program Manager.

\section{Mathematical Knowledge Manage- ment}

Bruce Miller

Abdou Youssef

Michael Kohlhase (Jacobs University, Bremen)

Heinrich Stamerjohanns (Jacobs University, Bremen)

See feature article, page 37.

\section{A Quality Pre-Processor for Biologi- cal Cell Images}

Adele Peskin

Karen Kafadar (Indiana University)

Alden Dima (NIST ITL)

Anne Plant (NIST CSTL)

John Elliot (NIST CSTL)

Michael Halter (NIST CSTL)

See feature article, page 31.

\section{Estimating Volumes of Simulated Lung Cancer Nodules}

\author{
David Gilsinn \\ Amelia Tebbe \\ Bruce Borchardt (NIST MEL)
}

Lung cancer is a disease of uncontrolled cell growth in tissues of the lung. It is the most common cause of cancer related deaths in men and second most in women and is responsible for an estimated 560,000 deaths in the US in 2007. Lung cancer lesions may be seen in Computerized Tomography (CT) images. CT is a medical imaging method that employs digital image processing techniques to generate three dimensional images from a large sequence of two-dimensional Xrays made around a single axis.

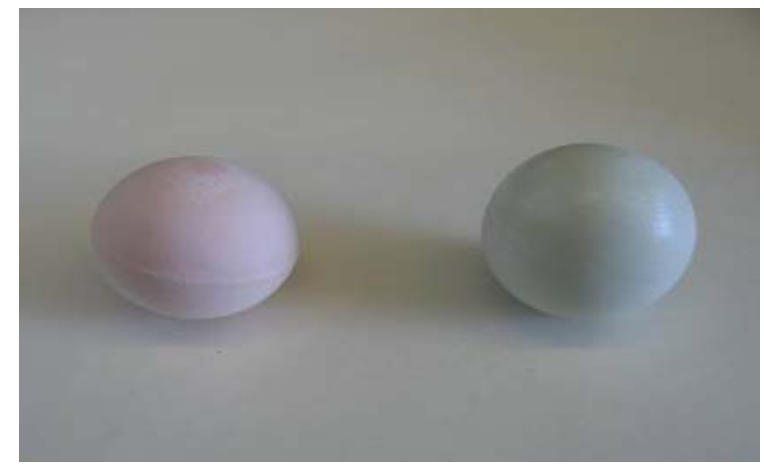

Figure 26. Physical phantoms used to simulate presence of lung cancers. Left: Pink phantom. Right: Green phantom.

The Food and Drug Administration (FDA) is conducting research on the development of reference cancer lesions, called phantoms, to test CTs and their underlying proprietary software. Two sample phantoms were loaned to NIST to estimate their volumes. The material of which the phantoms were composed simulates lung cancer material. The FDA can insert these phantoms into a simulated body torso for testing CT scanning and image reconstruction. The two phantoms are shown in Figure 26. Although they seem spherical they are slightly non-spherical. The phantom on the right in Figure 26 is referred to as Green and the one on the left is referred to as Pink.

The approach chosen to determine the volumes of the phantoms was based on a fundamental theorem in calculus, the Divergence Theorem. In this theorem a volume integral is shown to be equal to a particular surface integral. In order to develop a surface model of the phantoms, surface data was measured by a coordinate measuring machine (CMM) in the Manufacturing Engineering Laboratory (MEL) at NIST; see Figure 27. This machine produced a set of precisely measured $(x$, $y, z$ ) points on the surface of each phantom. As a reference, surface data was obtained on a well calibrated metal sphere in order to test the surface modeling process. In all cases, two sets of data points were measured, which we refer to as coarse (122 points) and dense (181 points). 


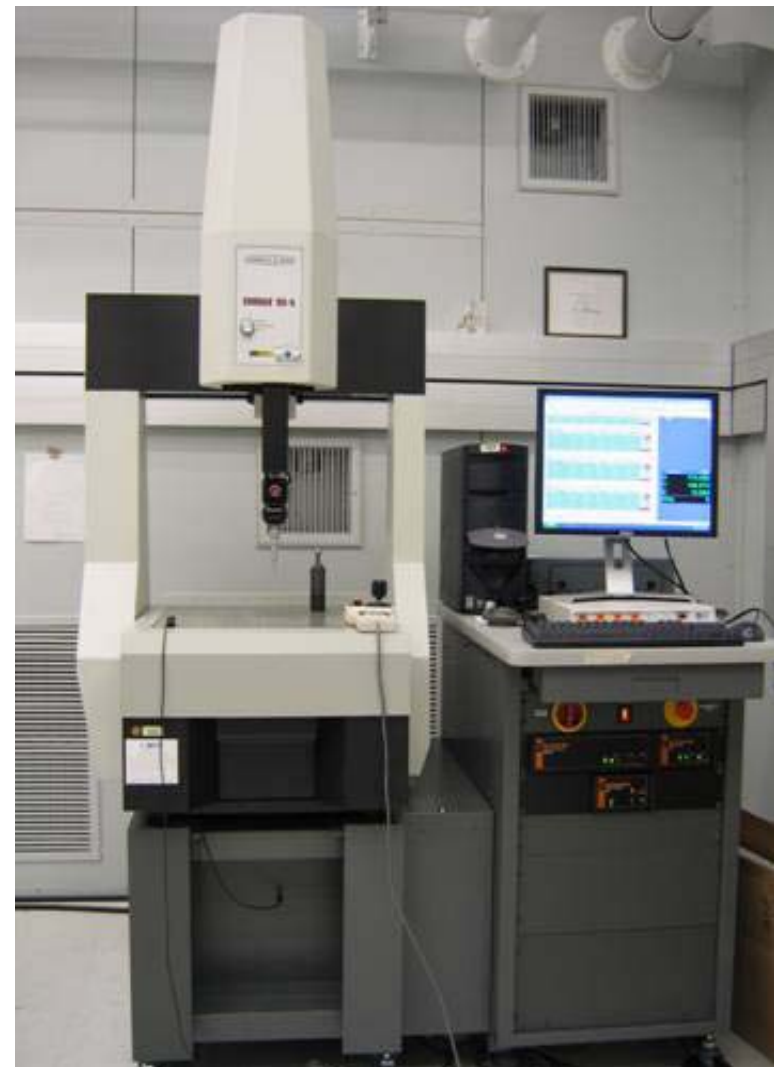

Figure 27. NIST Coordinate measureing machine.

The data were then transformed to spherical coordinates and modeled using a set of basis functions, called B-splines. The initial best fit, in the sense of least squares, of linear combinations of bivariate forms of the B-splines exhibited undesirable oscillations when the models were used to extrapolate values to points on the phantom surfaces. The least squares objective function was modified to include smoothing terms. This process, called regularization, produced very satisfactory extrapolation values at triangle vertices on the phantom surfaces. The surface triangulation was then used to compute the necessary surface integrals and finally the volumes using the Divergence Theorem.

In order to test the B-spline modeling process two approaches were taken to model the surface data from the calibrated metallic sphere. A spherical model was fit to the calibrated sphere by least squares parameter fitting and the B-spline model was fit to the sphere data using the regularization form of the objective function. The predicted volumes differed by approximately $0.01 \%$. This confirmed that the B-spline modeling process, using regularization, was a valid process and volume estimates of the phantoms would be acceptable.

As a check on the potential non-spherical shape of the phantoms a spherical model was fit to both of the phantoms. These produced volume estimates for the coarse and dense data from the Green phantom of $4331.3 \mathrm{~mm}^{3}$ and $4296.5 \mathrm{~mm}^{3}$ respectively. The expanded uncertainties for the center point and radius were estimated as order $10^{-1}$ and $10^{-2}$ respectively. For the Pink phantom the volumes were estimated as $3892.1 \mathrm{~mm}^{3}$ for the coarse data and $3912.5 \mathrm{~mm}^{3}$ for the dense data. The expanded uncertainties for the center point and radius were of order 1 and $10^{-1}$ respectively. This suggested a potentially spherical shape for the Green phantom and a slight non-spherical shape for the Pink phantom. When these results were estimated using the B-spline model, the volumes for the Green phantom were computed as $4331.8 \mathrm{~mm}^{3}$ and 4296.8 $\mathrm{mm}^{3}$ for the coarse and dense data respectively. Notice the closeness to the spherical model fit results. The expanded volume uncertainties were estimated as approximately $12 \mathrm{~mm}^{3}$ for the coarse data and $8 \mathrm{~mm}^{3}$ for the dense data. For the Pink phantom the volume results were $3854.7 \mathrm{~mm}^{3}$ and $3906.5 \mathrm{~mm}^{3}$ with expanded uncertainties of approximately $28 \mathrm{~mm}^{3}$ and $18 \mathrm{~mm}^{3}$. These differed slightly from the spherical model fit, again indicating a potential non-spherical form for the Pink phantom. The final report of this project has been submitted for publication in the Journal of Research of NIST.

[1] D. E. Gilsinn, B. R. Borchardt, and A. Tebbe, "Estimating Volumes of Simulated Lung Cancer Nodules," NISTIR 7571, July 2009.

[2] D. E. Gilsinn, B. R. Borchardt, and A. Tebbe, "Volume Estimation of Molded Artifacts by B-Splines," Proceedings of the SIAM Conference on Mathematics for Industry, 2009.

\section{Standardization of Lung Cancer Growth Measurements}

\author{
Adele Peskin \\ Javier Bernal \\ David Gilsinn \\ Alden Dima (NIST ITL) \\ Karen Kafadar (Indiana University)
}

Lung tumor malignancy is assessed by measuring the increase in tumor size over time in computed tomography (CT) scans. Current methods for estimating tumor size from CT scans are inaccurate, with results varying greatly from lab to lab. Standardized and accurate methods for estimating tumor volumes are needed. In order to assess the quality of volume measurements, we are preparing synthetic lung tumors of known volume for insertion into clinical CT data. In this regard, it is important that we reconstruct the complex geometries of real tumors that make them challenging to measure. 

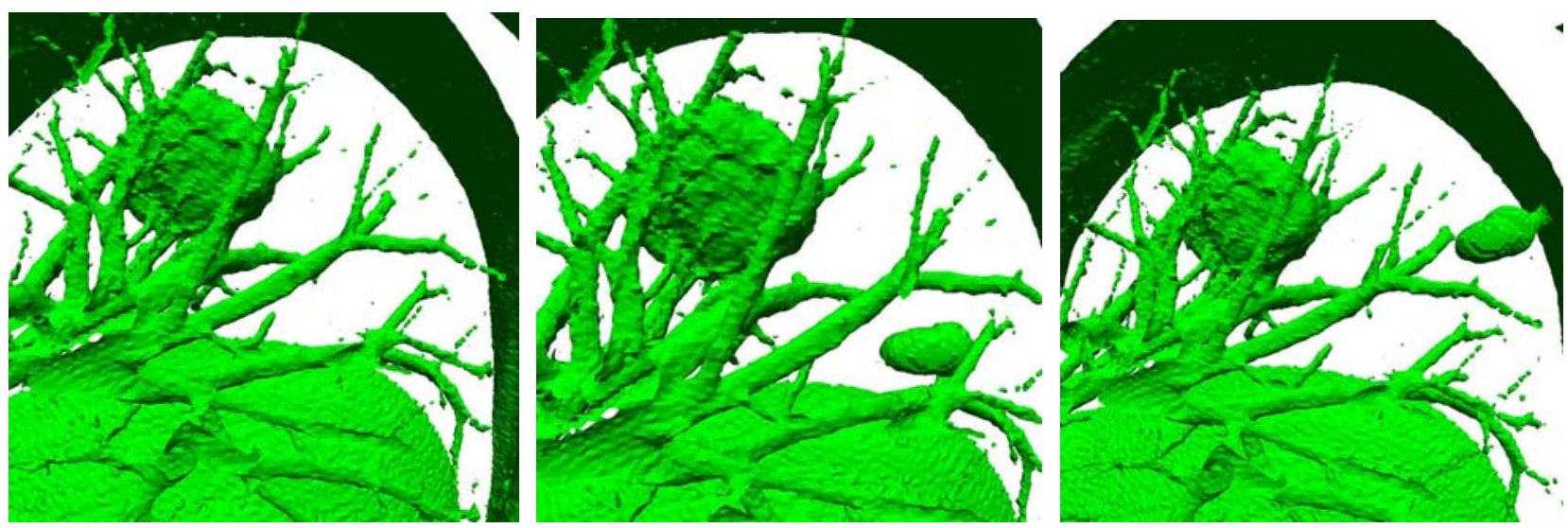

Figure 28. Isosurfaces of lung CT data taken at 400 Hounsfield units. From left to right: (a) The original lung CT data. (b) Synthetic tumor added at right. (c) Same synthetic tumor attached to the pleural lining of the lung using a cylindrical attachment.

Lung tumors are difficult to measure for a number of reasons. They are often small compared to the discretization of the CT data, so that large portions of the tumors only fill a fraction of the each voxel of the 3D data set, In addition, there are no standard methods to measure the partial volumes. Finally, the tumors are hard to separate from blood vessels that often surround them and from the pleural lining of the lung. By combining different geometric shapes, such as spheres, ellipsoids, cylinders, and cones, of different sizes and at different angles with respect to one another, we can recreate common shapes of lung tumor nodules inside clinical data at positions in the data that give rise to challenging volumetric issues. Several different tumor geometries are shown in Figure 28 that shows 3D isosurfaces of a region of lung data that contains both a clinical tumor (left) and several different synthetic tumors (right).

[1] A. Peskin, K. Kafadar, A. Dima, J. Bernal, and D. Gilsinn, "Synthetic Lung Tumor Data Sets for Comparison of Volumetric Algorithms," in Proceedings of the 2009 International Conference on Image Processing, Las Vegas, NV, July 13-16, 2009. 


\section{Complex Systems}

Complex Systems are composed of large interrelated, interacting entities which taken together, exhibit a macroscopic behavior which is not predictable by examination of the individual entities. The Complex Systems Program seeks to understand the fundamental science of these systems and develop rigorous descriptions (analytic, statistical, or semantic) that enable prediction and control of their behavior. Initially focused on the Internet and Grid Computing, this Program will facilitate predictability and reliability in these areas and other complex systems such as biotechnology, nanotechnology, semiconductors, and complex engineering. - Sandy Ressler, Program Manager

\section{Methods for Characterizing Com- plex Networks}

\section{Isabel Beichl \\ Brian Cloteaux \\ David Harris (NSA) \\ Francis Sullivan (IDA Center for Computing Sci.)}

Over the past decade, it has been realized that many of the networks inherent to natural and engineered systems share similar characteristics. These complex networks have been shown in systems such as the power grid, the Internet, communications and transportation networks, and software components. Since these networks are at the heart of our commercial infrastructure, it is important to develop a measurement science for such objects based on sound mathematical methods. Our project looks at both the modeling and measurement of complex networks of these types.

The development of predictive models of network characteristics is an important prerequisite. There have been a number of recent papers proposing possible mechanisms for explaining how complex networks could evolve. Unfortunately, the resulting models have not been able to realistically capture many of the structural characteristics that are observed in real life networks. Thus, we are currently unable to accurately model the time progression of complex networks. As an alternative, we have proposed a method for building random models through a completely structural approach [2].

This structural approach does not try to mimic how the network evolves, but rather it generates random networks with a series of characteristics that we choose as being important to our model. In order for us to produce dynamic models of these complex networks we have the additional constraint that our models must contain a core that reflects the network from an earlier time period. In other words, our method produces models that simulate the evolution of a network over time while maintaining certain critical characteristics, but without having to specify the exact generating mechanism. This approach has shown considerable promise in tests to predict the evolution of the autonomous system topology of the Internet.

A second research direction has been in the measuring of characteristics of these networks. One result has been in the area of computing the reliability polynomial of a network [3]. The reliability polynomial computes the probability that a network stays connected given that each edge in the network has an identical and independent chance of failure. Since it has been shown that this problem is computationally difficult, we have produced a new algorithm for approximating this polynomial. When compared to the best known published algorithm, our method has shown a significant speedup in its computation time.

We have also proposed an enhanced algorithm for computing reliability using a new importance function. A new technique for reducing variance is also being investigated. This technique involves adding a term to the estimator whose expected value is zero.

[1] I. Beichl and B. Cloteaux, "Measuring The Effectiveness of the s-Metric to Produce Better Network Models," in Proceedings of the Winter Simulation Conference (2008), Miami, FL, December 7-10, 2008, pp. $1020-1028$

[2] I. Beichl and B. Cloteaux, "A Structural Approach to the Temporal Modeling of Networks," in Proceedings of the 2009 Winter Simulation Conference.

[3] I. Beichl and B. Cloteaux, "An Approximation Algorithm for the Coefficients of the Reliability Polynomial," Congressus Numerantium, accepted.

\section{Standard Reference Data for Com- plex Network Research}

\author{
Roldan Pozo \\ http://math.nist.gov/ RPozo/complex_datasets.html
}

The study of complex or self-organizing networks is a relatively young, yet active research area. Much of the focus is to determine how graphs from real phenomena (such as protein interactions, social networks, structure of the World Wide Web, etc.) differ from synthetic or 
engineered graphs that are constructed by design. Clearly they are different, but how? Fundamental metrics, such as the clustering coefficient, graph diameter, and degree distributions, are commonly used in the literature, and these have served as encouraging first steps, but further research is needed to yield more meaningful distinctions and truly understand the nature of complex networks.

One important question to ask is: what are the precise real graphs that are being used to drive these theories? Clearly, the conclusions being reached can be only as meaningful as the quality and availability of the original data. Most papers provide little such information, making comparisons or verification of approaches difficult. For example, a commonly studied collaboration network in the literature is the Movie Actor graph, where two actors are connected if they have worked together on the same movie. While, this information is usually culled from the Internet Movie Database, there is no single Movie Actor graph because this database is a changing snapshot of the entertainment industry at any given time. Furthermore, what one chooses to include in the search (e.g., foreign films, documentaries, TV movies, specific epochs or genres) will generate different graphs. In fact, there are several versions of the Movie Actor graph cited in the literature, so a citation is not meaningful unless it contains a time-stamp and a record of the specific queries used. A similar argument can be made for other association networks, such as co-author or citation graphs.

Another example is networks based on the structure of the World Wide Web. In this case, each web page is a node and two pages are connected if there a hypertext link from one to the other. These are typically generated by web crawlers and can vary greatly depending on where they crawl and what decisions they make about what constitutes a link. For example: Do different anchors to the same page count as separate links? How are non-static pages handled? What file types are processed? How robust it is with broken links and sloppy HTML pages? This list can be quite long, and each decision path will yield a different graph. Aside from this, the Web is, of course, not a static entity. Many of the Web graphs cited provide little of these details and usually present only the topological structure (i.e., URLs removed) so it is difficult to confirm or validate them independently.

To address these issues, we have developed and deployed our own set of web crawlers that report not only topological structure, but also record the node URLs for verification and validation, as well as gener- ate a unique checksum to ensure that pages which contain identical material (e.g., aliased, or mirrored pages) are uniquely identified. Furthermore, these web crawls can be fined-tuned to record and characterize data along a precise decision path, thus allowing us to see what differences are generated when we alter the definition of what is a link and what is a node.

Similar concerns exist for graphs taken from other application domains. Often the data is incomplete, poorly documented, or have missing metadata crucial to its characterization. There are ongoing debates about whether particular networks are really scale-free or not, or whether the conclusions of some papers are actually valid. Usually these concerns lie at the heart of how data was collected and analyzed.

To help improve this situation we are developing standard reference data sets for complex networks. This collection of public and well-documented "realworld" graphs will serve as a test bed of reasonably validated data which researchers can utilize to

1) verify and compare the algorithms and analysis of various approaches in the literature,

2) ensure that everyone is using the same reference data in their studies,

3) have one convenient location where one can browse and search for network graphs with various characteristics,

4) provide a focal point for the research community to contribute and exchange network graphs from various application domains, and

5) provide a testbed for development of software analysis tools (e.g., viewers, partitioners, clustering algorithms) that will aid in the further development and research of complex networks.

In further aiding the understanding of such network systems, we have developed a set of software tools for working with mathematical graphs (Ngraph++) and processing and transforming data collections into various formats (e.g., Matrix Market, GraphViz, Matlab) for output and analysis. Ngraph++ computes clustering coefficients and degree distributions for directed/undirected graphs. Although a small and simple library, it provides intuitive graph operations (subgraphs, unions, differences) and complex-network features not commonly found in more complex packages. Together with various Python and scripting tools, and a reference dataset of network graphs, this forms a kernel package for analyzing complex network data in a portable and universal fashion. 


\section{Community Detection in Network Graphs}

\author{
Roldan Pozo \\ Clement Rey
}

Detecting community structure in large graphs is one of the fundamental challenges in network science. This line of research probes at the underlying structure of complex systems, and is necessary in understanding how such systems behave over time. A community is loosely defined as a subset of nodes which are highly connected to each other, but less connected to the remaining graph. Identifying such node groups is important in understanding graph evolution, synchronization, and the dynamics of networks. In various application contexts, community detection can classify customers with similar purchasing habits, determine graph layouts for visualization, model the spreading of infectious diseases, and help in identifying crime cells.

Extracting such structure reliably from large-scale networks, however, is a difficult problem. First, there must be some mathematical metric for what constitutes a graph community; secondly we need an algorithm for finding such communities (related solutions are NPcomplete); lastly, we have to have some way of verifying that the computed groups are indeed meaningful. Various metrics have been proposed and several optimization algorithms introduced in the literature.

The fundamental question remains: How well do these approaches work in practice? So far, there has been little work in verifying and validating the results on a wide array of application domains. To aid this study, we are currently investigating eigenvalue-based methods and fast greedy algorithm approaches which run in $O\left(n \log ^{2} n\right)$ for sparse graphs, and studying their behavior on specific data sets for which we have ground truth community information available. Initial results suggest that current state-of-the-art algorithms provide only mixed results and further research is needed to provide truly reliable solutions.

\section{Visualization of Large Complex Networks}

\section{Roldan Pozo \\ Clement Rey \\ Terence Griffin}

One of the fundamental challenges in complex systems is to understand the nature and structure of large-scale networks. These can be information networks (such as the World Wide Web) biological networks (like protein-protein interactions, food webs) social networks

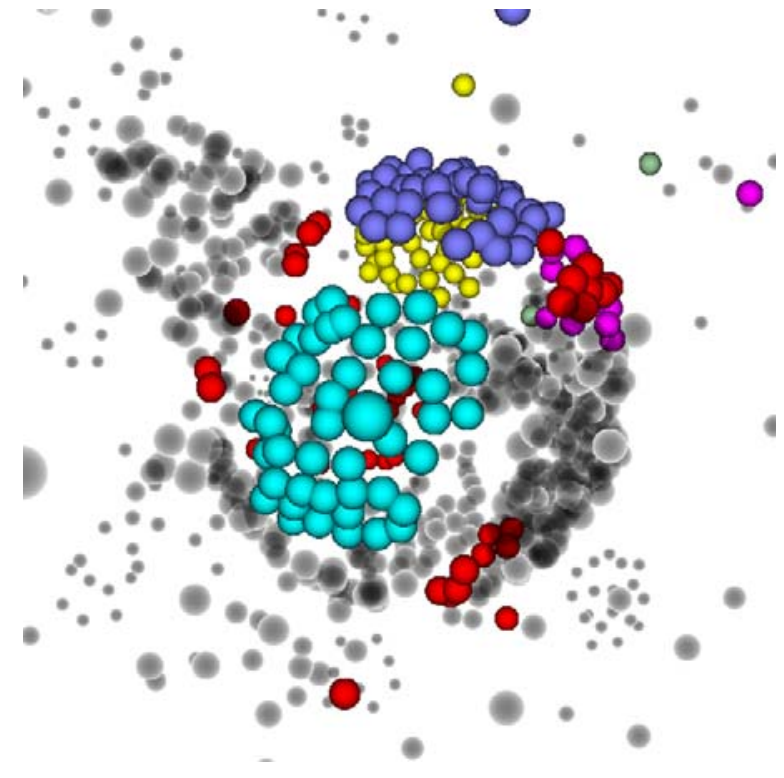

Figure 29. $3 D$ visualization of community structure of the World Wide Web graph of the George Washington University Computer Science Department (www.cs.gwu.edu). Each node is a separate web page and colors indicate different community groups. The transparent grey color indicates the subset of very small groups.

(e.g., online social sites, personal interactions, criminal rings, terrorist cells) and engineered networks. Such networks typically exhibit thousands to millions of nodes and about 10 times as many edges. Given the rapid growth of electronic data, networks with billions of nodes soon will be readily available for analysis, providing greater insight into the behavior of largescale systems.

To the aid the understanding of such systems, we have developed visualization techniques that utilize the latest immersive and 3D environments, together with state-of-the-art graph layout algorithms, so that researchers can walk around and examine these structures interactively. The visualizations can also be run on independent desktops.

For example, Figure 29 is a visualization of a web graph, were nodes are individual web pages of George Washington University. In this instance, we have highlighted the community structure by using a fatpoint shader (with an interactive size control) to render the nodes of the graph. The largest groups ( 7 in this case) are colored individually. The set of small groups (179 in this case) is colored a transparent grey. An individual node can be selected using a cross-hair glyph and its URL displayed in a box tethered to the node with lines. In addition, the web page that the node represents can be brought up, so researchers can investigate its content. 


\section{Fluid-level Modeling of TCP}

\section{Vladimir Marbukh \\ Daniel Genin (NIST ITL)}

While modeling of Transmission Control Protocol (TCP) at the packet level yields accurate results, its computational complexity makes packet-level modeling infeasible even for moderate size networks. Flowlevel models allow for significant reduction in computational and simulation complexity at the cost of loss in accuracy. Conventional flow-level models [1], which assume that at equilibrium each flow has fixed rate, do not take into account TCP flow burstiness. This simplifying assumption results in underestimation of the packet loss probability and thus overestimation of the TCP throughput which is inversely related to the packet losses. Quantification and mitigation of this inaccuracy is an open issue of significant practical and theoretical importance due to the central role played by TCP in the Internet.

In [2] we assess that conventional fluid models [1] result in unacceptably high underestimation of the numbers of flows in progress and file transfer times in TCP-controlled network under arriving/departing flows. To alleviate this deficiency [2] proposes modeling TCP at flow-level by an on-off fluid process [3]. Model [3], often named the "AMS model" after authors D. Anick, D. Mitra and M. M. Sondhi, accounts for the flow burstiness while retaining the computational tractability. Our extensive $n s 2$ simulations [2] suggest that the AMS flow-level model of TCP may have substantially better accuracy than the conventional fluid model with fixed rate [1], potentially leading to faster yet accurate Internet simulation. More efforts are needed to improve accuracy of the proposed AMS model under high load by accounting for the TCP slow start phase.

[1] R. Srikant, Mathematics of Internet Congestion Control, Willey, 2001.

[2] D. Genin and V. Marbukh, "Bursty Fluid Approximation of TCP for Modeling Internet Congestion at the Flow Level," Forty Seventh Annual Allerton Conference on Communication, Control, and Computing, Monticello, Illinois, USA, 2009.

[3] M.M. Sondhi D. Anick, D. Mitra. Stochastic theory of a data handling system with multiple sources. ICC'80; International Conference on Communications, Seattle, USA, 1980.

\section{Analysis of a Distributed Protocol for Network Control}

Fern Y. Hunt

Vladimir Marbukh

This year we made progress in studying the properties of a discrete dynamical system that models TCP congestion protocol with multipath routing. Here, traffic is split over several routes according to a probability distribution that is constrained by an entropy parameter. This parameter measures the robustness or path diversity of the splitting scheme. F. Kelly and others have observed that many protocols currently in use can be viewed as distributed algorithms for solving an optimization problem. In two sample network topologies we show that the corresponding discrete system does tend to a stable equilibrium that is the solution of a network utility optimization problem when the entropy of the route distribution is greater than a critical value. This value is a function of the network link capacities and network topology. For our model we show that there is no stable equilibrium for entropy values less than the critical value. A NIST Internal Report describing this research has been released [1].

The existence of an explicit parameter measuring path diversity allows us to evaluate the trade-off between path robustness and network utility. We looked again at the two sample networks which in both cases have a single source and destination. We computed the the long term average utility as a function of entropy. Starting with the critical value, as the entropy increases, the network utility (which we show depends on the minimum average or mean cost) decreases. Thus increased robustness leads to decreased utility. This work is also described in the internal report [1] and in a later paper that focuses on the tradeoff between utility and stability [2].

[1] F. Hunt and V. Marbukh, "A Model of Joint Congestion Control and Routing Through Random Assignment of Paths," NIST Interagency Report 7590.

[2] F. Hunt and V. Marbukh, "Measuring the Utility/Path Diversity Trade-Off in Multipath Protocols," Proceedings of the Fourth International ICST Conference on Performance Evaluation Methodologies and Tools, ValueTools09, Pisa, Italy. 


\section{Visualization of Possible Route Probability Distributions}

\section{Fern Y. Hunt}

Terence Griffin

In order to control congestion and maximize network utility, a variety of routing protocols can be used to allocate network traffic. When multiple paths linking a source and destination are available, but only a single path, e.g., a shortest path or path of minimum cost, is selected, we have the Open Shortest Path First (OSPF) protocol. On the other hand, traffic could be allocated uniformly when the paths have the same cost. A spectrum of path specification strategies lies in between these extremes. We visualize these possibilities in the case of a single source destination pair with 3 possible connecting paths. If $\beta_{1}, \beta_{2}$, and $\beta_{3}$ are the fractions (probabilities) of traffic assigned to paths 1,2 , and 3 respectively, then each allocation can be associated with a point in or on the equilateral triangle with an altitude height of 1 . Here, $\beta_{1}, \beta_{2}$, and $\beta_{3}$ are the lengths of perpendiculars from points on edges of the triangle. The entropy associated with an allocation is a function of $\beta_{1}, \beta_{2}$, and $\beta_{3}$, and provides an important quantitative description of its degree of randomness. A point in the triangle is colored according to the value of the entropy of the allocation represented by that point. The surface above the triangle was created by plotting a point above the data point in the triangle where the height is equal to the Entropy. OSPF is associated with $\mathrm{H}=0$, and traffic is allocated according to one of the vertices of the triangle. This is the least robust allocation. Equal cost multipath allocation, assigned to the center of the triangle has the maximum value of $\mathrm{H}=\log (3)$ is the most robust but it has reduced network utility. (See the magenta dot in the center of the triangle). Bands of points with the same value of $\mathrm{H}$ (up to accuracy .001) are given the same color. As $\mathrm{H}$ decreases, an oval emerges from the center and grows until it touches the sides of the triangle at the maximum entropy for a network with 2 paths (depicted by the magenta oval in the triangle and band in the surface above it). Theoretical studies predict a change in network dynamics for $\mathrm{H}$ smaller than this critical value.

Our visualization runs immersively and on the desktop. Entropy is displayed as the third dimension and uses an RGB color ramp on entropy. The visualization allows selection of entropy values, and groups of related points. The structure can also be examined with clipping planes of various types, and also by selection of two dimensional or three dimensional representations. The data can be displayed in three ways: points with fatpoint shaders with adjustable point size; polygonal surface; and wireframe surface.
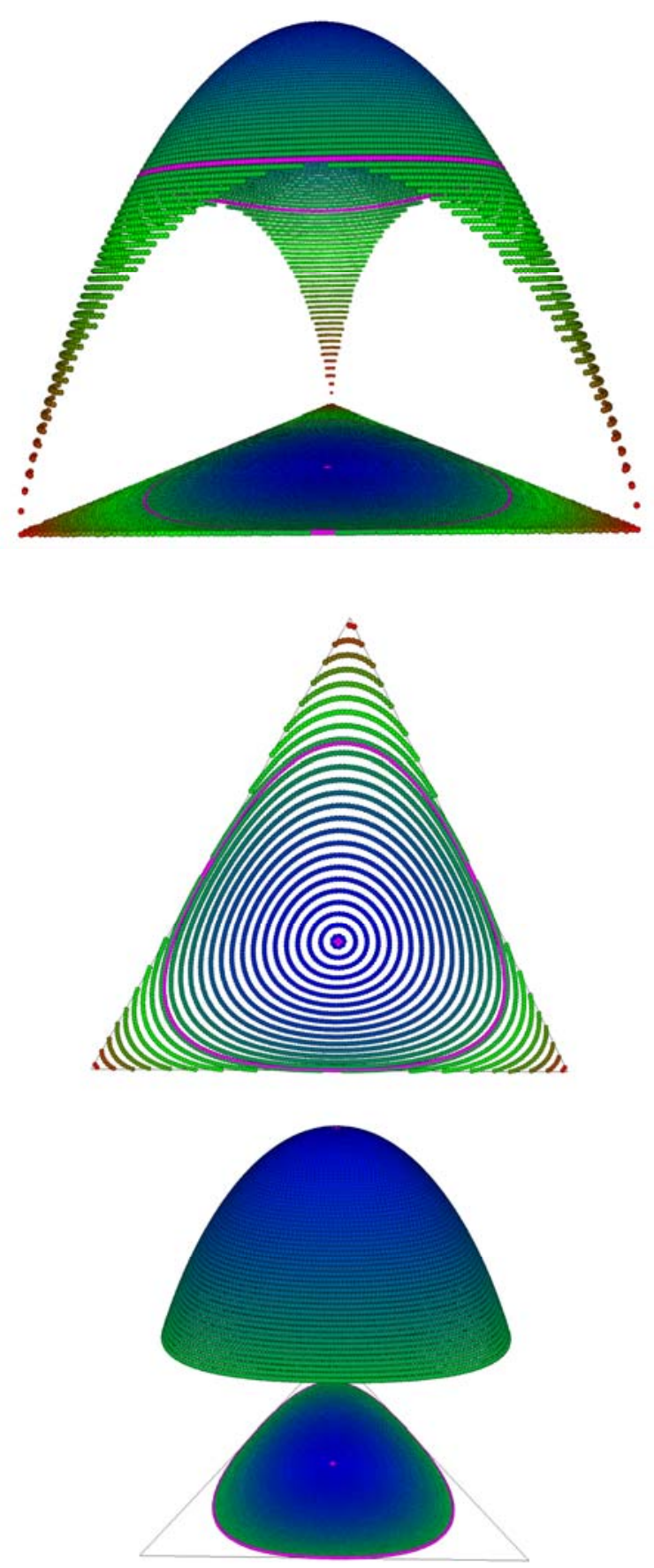

Figure 30. This figure shows the surface over the triangle defined by the equation: $H\left(a^{1}, a^{2}, a^{3}\right)=-a^{1} * \log \left(a^{1}\right)-a^{2} \log \left(a^{2}\right)$ $a^{3} \log \left(a^{3}\right)$, where the height of a point on the surface above the triangle is the value of $H$ for the point in the triangle representing $\left(a^{1}, a^{2}, a^{3}\right) . H$ is the entropy function. The top of the surface is the maximum value of the entropy, i.e. $\log 3$ which corresponds to the point $(1 / 3,1 / 3,1 / 3)$ whose image in the triangle is the pink dot in the center. The surface illustrates the changes in the level curves of $H$ (i.e. points in the triangle with a constant entropy value) as $H$ decreases from $\log 3$ to 0 . Colors indicate different values of entropy. On the left is the fatpoint shader representation. On the right (top) are two dimensional and polygonal (bottom) representations. 


\section{Grid Network Simulation Using Piece-Wise Homogeneous Markov Chains}

Fern Y. Hunt

Christopher A. Dabrowski (NIST ITL)

The growing use of services provided by cloud and grid computing networks underlines the need for analytical and computational tools for understanding and predicting the behavior of such complex systems. Our work centers on the analysis of grid networks. Here the challenge is that under decentralized control, the interactions of many service providers and customers could result in emergent behaviors resulting in unpredictable and undesirable outcomes. A straightforward approach is to perform a discrete event simulation to model customers and service providers entering into service level agreements for a variety of computer services. Simulations that seek to accurately reproduce system structure and component behavior are computationally expensive however. Even simulating an entire day takes many hours of CPU time (see [4]). As analysts seek to identify system execution paths and scenarios that lead to system breakdown and other anomalous behavior, there is a critical need for models that can provide rapid scalable simulation of large scale grid systems. One should also be able to quickly perturb these models in a search for such scenarios.

In the previous year, [2,3], we developed a piecewise homogeneous Markov chain model of a grid network system that compared very favorably with discrete event simulations (i.e., large-scale simulation) including several failure scenarios arising from the perturbation of transfer rates in the grid system. Moreover, the Markov chain simulation produced these results in hours while the large-scale simulation required days of processing.
This year, we took another step in our goal, introducing an analytical approximation of the Markov chain that eliminated the need for simulation when computing the task completion rate. These computations are much faster than Markov chain simulation. Secondly we introduced a method for finding failure scenarios in the Markov chain model based on identifying cut point sets in the graph of the Markov chain. Identifying the most critical cut sets is an alternative to the brute force perturbation method used in [2,3], and in a forthcoming paper, we demonstrate that a heuristic node contraction algorithm can very quickly identify many of these sets in some sample large graphs. The results of this algorithm compare quite favorably with the algorithm developed by Tsukiyama et al. that performs a complete enumeration of all cutsets. The speed of the node contraction algorithm relative to the algorithm of Tsukiyama et al. depends on the connectivity of the graph. In the examples tested, node contraction is faster on graphs with moderate connectivity but it is dramatically slower when graph connectivity is high. We are currently developing a report on our work this year [1].

[1] C. Dabrowski, K. Morrison, and F. Hunt, "A Fast and Scalable Representation of Grid Network Failure," in preparation.

[2] C. Dabrowski and F. Hunt, "Markov Chain Analysis for Large-Scale Grid Systems," NIST Interagency Report 7566.

[3] C. Dabrowski and F. Hunt, "Using Markov Chain Analysis to Study Dynamic Behavior in Large-Scale Grid Systems," in Proceedings Seventh Australasian Symposium on Grid Computing and e-Research (Ausgrid2009), Wellington, New Zealand, CRPIT,99, P. Roe and W. Kelly, Eds. ACS, pp.29-40.

[4] K. Mills and C. Dabrowski, "Investigating Global Behavior in Computing Grids," in Self Organizing Systems, Lecture Notes in Computer Science, volume 4124, Springer-Verlag, pp. 120-136. 


\section{Quantum Information}

An emerging new discipline at the intersection of quantum mechanics and computer science, quantum information science $(Q I S)$ is likely to revolutionize science and technology in much the same way that lasers, transistors, electronics, and computing did in the $20^{\text {th }}$ century. At the very least, QIS has the potential to provide phenomenal increases in information storage and processing speed and communication channels with extremely high levels of security guaranteed by the laws of physics. However, the needed manipulation and control of quantum states remains a grand challenge. We are developing theoretical underpinning to support the multi-laboratory experimental program in quantum information at NIST. This work is aimed at the development of a measurement science for quantum information processing systems. - Ronald Boisvert, Program Coordinator

\section{Restrictions on Transversal Encoded Quantum Gate Sets}

\author{
Bryan Eastin \\ Emanuel Knill
}

See feature article, page 21.

\section{Quantum Computing Theory}

\author{
Bryan Eastin \\ Scott Glancy \\ Emanuel Knill \\ Adam Meier \\ Mike Mullan \\ Yanbao Zhang \\ R. Somma (Perimeter Institute and LANL) \\ S. Boixo (California Institute of Technology) \\ G. Ortiz (University of Indiana)
}

Quantum computing involves the exploitation of quantum mechanical effects to improve our ability to solve computational problems such as factoring and physics simulation. Our contributions to quantum computing theory include work on better understanding the limitations as well as the power of quantum computers.

To improve our ability to exploit the advantages of quantum computing, we are working on better understanding and applying adiabatic processes in algorithms. An adiabatic process normally requires slowly varying the parameters of the Hamiltonian determining the evolution of a quantum system. An initial ground state is thus induced to follow the ground states of the changing Hamiltonian. In this way one can prepare complex states from simple initial states. The conditions for this following are quite subtle, but we found that by randomizing the evolution and making energy measurements, the desired state preparation can be performed more efficiently, with the conditions having a simpler dependence on the parameters.
The advantages of quantum computing are easiest to see in the context of so-called oracle problems. In these problems, we are given an oracle or "black box" that we can apply to a quantum register. The task is to infer a property of the oracle, such as which computational basis state's phase it changed (this is the "search" problem). For the purpose of proving advantages, one must determine upper bounds on the probabilities of success of classical algorithms and compare them to probabilities of success of quantum algorithms. Perhaps the most powerful general technique for this purpose is called the "adversary method." An exact version of this method can be based on semidefinite programming, and we have been investigating its application to determine exact optimal success probabilities of both classical and quantum algorithms for small instances of oracle problems. An important generalization that we are developing involves oracles that interpolate between quantum and classical by containing varying amounts of decoherence. We intend to apply this to characterize the power of experimentally available quantum registers.

One of the often-mentioned features of spatially separated quantum systems is that they have states that cannot be described with so-called local realistic models in terms of independent states of each system, as one might expect to be able to do classically. Violation of local realism can be detected from the statistics of independent measurements of the systems. By making such measurements, one can obtain very strong security guarantees for protocols such as secret key exchange. Experiments that demonstrate such violations have been performed, but have one or more loopholes, the best known of which are the "detection loophole" (introduced by the inefficiencies of typical quantum measurements) and the "locality loophole" (associated with the possibility of hidden communication between the quantum systems consistent with relativistic causality). To help close the detection loophole, we previously initiated a study of an experimental configuration involving photon sources to determine tradeoffs between detector inefficiency, source parameters, and the rate at which confidence in 
violation is gained. We have now completed this work. A possible loophole not often considered is introduced by possible dependencies of each data point on what happened before in an experiment. This is called the memory loophole. Previously, several researchers have pointed out that it can be closed by a more sophisticated statistical analysis of the data based on martingale inequalities from probability theory and statistics. We found that a better and asymptotically optimal approach can be derived from prediction-based likelihood ratios. This is insensitive to experimental stability, possibly simplifying experimental control.

Violations of local realism, in the sense just described, appear not to be necessary for quantum speedups over classical computation. We are investigating whether a different kind of non-classicality can be shown to be necessary for the efficient solution of any classically intractable problem. We have demonstrated that this is indeed the case for quantum computations composed of two-qubit gates, though a similar result may not hold for more general gate sets.

[1] S. Boixo, E. Knill, and R. D. Somma, "Quantum State Preparation by Phase Randomization," arXiv: 0903.1652, Journal of Quantum Information and Computation 9 (2009), pp. 833-855.

[2] E. Knill, "Quantum Computing Q\&A," in review.

[3] R. Somma, S. Boixo, and E. Knill, "Measurement-Based Quantum Computation," in preparation.

[4] Y. Zhang, E. Knill, and S. Glancy, "The Statistical Strength of Experiments to Reject Local Realism with Photon Pairs and Inefficient Detectors," in review.

\section{Fault-tolerant Quantum Computa- tion}

\section{Bryan Eastin \\ Emanuel Knill \\ Adam Meier}

Advances in the theory of fault-tolerant quantum computation are important for the development of quantum computers as the hardware employed in the first generation of such devices is likely to be highly prone to error. The theory of fault tolerance addresses the problem of implementing encoded gates and error correction in a way that precludes a catastrophic failure from resulting from the malfunction of a few isolated components. This can be achieved by preventing errors from spreading within individual blocks, i.e., within independent error-correcting units, of a quantum code.

Previously, we settled a long-standing question in quantum information theory, namely, whether there exist nontrivial quantum codes for which all logical gates can be implemented transversally. Transversal encoded gates never cause subsystems within a code block to be coupled and are, consequently, fault tolerant. Their simplicity and robustness makes transversal encoded gates valuable tools for quantum computation, but, for all known codes, such gates are inadequate for achieving full computational universality. We showed that no nontrivial transversal encoded gate set could be universal.

We have also begun investigating new procedures for magic-state distillation, a technique for improving the quality of prepared magic states. At present, the most commonly considered method of achieving universal quantum computation is through (magic) state injection and distillation. Magic states are those that, given the ability to perform Clifford gates (a class of operations that are frequently amenable to being made fault tolerant), can be both distilled and used to perform universal quantum computation. Though it is a widely known technique, many interesting questions regarding magic state distillation remain. We hope to begin to address some of these by developing a general theory of magic state distillation. In addition, we plan to calculate the output error probability for a novel distillation procedure that we have already developed and believe to have some superior qualities with regard to resource usage.

Finally, we have made some progress on extending the techniques of DiVincenzo and Aliferis for using unverified ancillae in quantum error correction and measurement. They showed that a fault-tolerant scheme based on concatenating the 7-qubit Steane code could be implemented, with little additional overhead, using unverified ancillae and extremely slow measurements. We have extended their result to faulttolerant schemes based on a variety of other codes and hope to further expand the range of applicability of this intriguing technique.

In addition to continuing our work on state preparation, we plan, in the coming year, to investigate techniques for passive quantum error correction. Specifically, we plan to study the efficacy of proposed passive quantum error correcting architectures and to seek new architectures supporting passive correction. The success of any of these research projects could contribute to the goal of constructing a quantum computer by reducing the overhead and/or precision required.

[1] B. Eastin and E. Knill, "Restrictions on Transversal Encoded Quantum Gate Sets," arXiv: 0811.4262, Physical Review Letters 102 (2009), 110502. 


\section{Quantum Computing Benchmarks}

\author{
Emanuel Knill \\ Bryan Eastin \\ Adam Meier \\ Michael Mullan \\ Dietrich Leibfried (NIST PL) \\ David Wineland (NIST PL)
}

Quantum computers promise to greatly speed up the solutions to some problems in mathematics and physics. However, there are substantial obstacles to our building quantum computers. Today, we are able to see quantum effects with up to 8 quantum bits (qubits), but barely able to properly compute with two. We are working on ways to consistently determine the amount and quality of experimentally available quantum resources by developing standard, device-independent benchmarks and participating in experiments to realize them, particularly in ion-trap quantum information processing systems.

In previous years we developed and implemented randomized benchmarking strategies for one qubit, a strategy that has now been tried with nuclear magnetic resonance and superconducting qubits. We are currently working on extending this strategy to more qubits. The basic idea is to perform experiments with many random sequences of so-called Clifford gates and compare the measurement outcomes with the ones predicted from simulations. The results are processed to infer an effective probability of errors for each step of a typical computation. Clifford gates allow efficient prediction of measurement outcomes while being sufficiently powerful to enable universal quantum computing given the additional capability to prepare a special but robust state. We have the necessary simulation capabilities and developed principles for randomizing multi-qubit gates and inferring not only overall error probabilities, but given enough data and a simplifying assumption, the error behavior of each type of gate. Verification of the principles are in progress and possible ion-trap experiments are in the planning stages.

Part of this project is to develop measurement strategies for quantifying the amount of quantum resources contained in a given experimental quantum information processing device. One approach is to obtain standard criteria for when one can claim to have a one, two or more qubit quantum register. One of the criteria should involve demonstration of a quantum advantage, namely that the device can do better than any quantum register of smaller size. Randomized oracle problems, such as the search problem, can be used for this purpose, but to do so requires a rigorous way of comparing not only classical and quantum algorithms, but algorithms that use restricted quantum resources. We anticipate using the semidefinite programming techniques developed in the course of the Quantum Computing Theory project for this purpose.

\section{Quantum State Estimation Theory and Applications}

\author{
Scott Glancy \\ Emanuel Knill \\ Bryce Calkins (NIST EEEL) \\ Tracy Clement (NIST EEEL) \\ Thomas Gerrits (NIST EEEL) \\ Adriana Lita (NIST EEEL) \\ Alan Migdall (NIST PL) \\ Richard Mirin (NIST EEEL) \\ Sae Woo Nam (NIST EEEL) \\ Gene Hilton (NIST EEEL) \\ Kent Irwin (NIST EEEL) \\ Konrad Lehnert (NIST PL) \\ Leila Vale (NIST EEEL)
}

Many emerging technologies will exploit quantum mechanical effects to enhance metrology, computation, and communication. Developing these technologies requires improved methods to measure the state of quantum systems. Quantum state estimation is a statistical problem of estimating an underlying quantum state using a collection of measurements made on independently prepared copies of the state. Accurate quantum state estimation allows experimentalists to answer the questions "What quantum state does my device prepare?" and "How confident am I that it has prepared that state?" In the last year we have made several improvements to the algorithms used to answer these questions. These include a criterion for stopping iterations of the estimation algorithm and a new iterative algorithm with faster convergence. We have also implemented a parametric bootstrap method to assign uncertainties in the quantum state estimate and discovered that conventional algorithms for quantum state estimation are biased toward states of lower purity.

We have been applying quantum state estimation in two experiments. The first of these is an experiment to prepare Schrodinger cat states of light, which is part of the IMS project "Quantum Optical Metrology with NPhotons." This experiment uses high efficiency transition edge sensors to subtract three photons from a pulse of squeezed vacuum light. The photon subtraction prepares the Schrodinger cat state, which is measured by homodyne detection. Our quantum state estimation software is used to reconstruct the state from the measurements. Our state estimation algorithm corrects for the photon loss and electronic noise of the homodyne detector to infer the originally created state. The state 
created in the laboratory has a fidelity of $0.59_{-0.14}^{+0.04}$ with an ideal Schrodinger cat state with a mean of 3.08 photons.

The second experiment where we have been applying quantum state estimation is the DARPA funded collaboration with JILA titled "Analog Quantum Integrated Circuits." The goal of this project is to create entangled states in superconducting microwave circuits and to use that entanglement for quantum teleportation. We have been developing methods to calibrate the amplifiers used to measure the quantum states' of microwaves. We have also used our quantum state estimation software for preliminary analysis of squeezed states of microwaves.

[1] S. Glancy, E. Knill, T. Gerrits, T. Clement, B. Calkins, A. Lita, A. Miller, A. Migdall, S. W. Nam, and R. Mirin, "Innovations in Maximum Likelihood Quantum State Tomography," Perimeter Institute for Theoretical Physics Quantum Information Seminar, Perimeter Institute, Waterloo, Canada, September 9, 2009.

[2] T. Gerrits, S. Glancy, T. Clement. B. Calkins, A. Lita, A. Miller, A. Migdall, S. W. Nam, and R. Mirin, "Generation of Optical Schrodinger Cat States by Numberresolved Photon Subtraction from Squeezed Vacuum," in preparation. 


\section{Virtual Measurement Systems}

A virtual measurement is a quantitative result and its uncertainty, obtained primarily by a nontrivial computer simulation or computer-assisted measurements. Examples of virtual measurements include computational models of physical systems and visualizations of the results. The Virtual Measurement Systems Program introduces metrology constructs - standard references, uncertainty characterization and propagation, and traceability - into scientific computation and computer-assisted measurement technologies. As with physical measurement systems, development of a virtual metrology infrastructure will result in predictive computing with quantified reliability. In turn, this will enable improved decision making contingent on virtual measurement systems. - Andrew Dienstfrey, Program Manager

\section{High Precision Calculations of Fundamental Properties of Few- electron Systems}

\author{
James Sims \\ Stanley Hagstrom (Indiana University)
}

See feature article, page 25.

\section{Micromagnetic Modeling}

\author{
Michael Donahue \\ Donald Porter \\ Robert McMichael (NIST CNST) \\ June Lau (NIST MSEL)
}

\section{http://math.nist.gov/oommf/}

Advances in magnetic devices such as recording heads, field sensors, magnetic nonvolatile memory (MRAM), and magnetic logic devices are dependent on an understanding of magnetization processes in magnetic materials at the nanometer level. Micromagnetics, a mathematical model used to simulate magnetic behavior, is needed to interpret measurements at this scale. MCSD is working with industrial and academic partners, as well as with colleagues in NIST CNST, MSEL, and EEEL, to improve the stateof-the-art in micromagnetic modeling.

Michael Donahue and Donald Porter have developed a widely used public domain computer code for doing computational micromagnetics, the ObjectOriented Micromagnetic Modeling Framework (OOMMF). OOMMF serves as an open, welldocumented environment in which algorithms can be evaluated on benchmark problems. OOMMF has a modular structure that allows independent developers to contribute extensions that add to the basic functionality of OOMMF. OOMMF also provides a fully functional micromagnetic modeling system, handling both two and three-dimensional problems, with so- phisticated extensible input and output mechanisms. In fiscal year 2009 alone, the software was downloaded more than 5,200 times, and use of OOMMF was acknowledged in more than 120 peerreviewed journal articles. OOMMF has become an invaluable tool in the magnetics research community.

Key developments in OOMMF last year include:

1) Addition of multi-threaded computation in the modules of OOMMF for use on multiprocessor, multi-core computers, enabling faster (observed speed-up of four to seven times) and larger simulations, and more efficient utility of common computing platforms [2].

2) Creation of additional modules tailored for the simulation of polycrystalline materials, common in practical device designs [3].

OOMMF is part of a larger activity, the Micromagnetic Modeling Activity Group ( $\mu \mathrm{MAG})$, formed to address fundamental issues in micromagnetic modeling through two activities: the development of public domain reference software, and the definition and dissemination of standard problems for testing modeling software. MCSD staff members are involved in development of the standard problem suite as well. There are currently four standard problems in the suite, testing both static and dynamic magnetization properties. A new standard problem proposed jointly by several authors [1] aims at validation of simulations of spin momentum transfer in the "current parallel to plane" geometry. We are in the process of publishing this proposed problem as the fifth $\mu \mathrm{MAG}$ standard problem. Work continues to develop additional standard problems to address the spin momentum transfer phenomenon in other geometries.

[1] M. Najafi, et al., "Proposal for a Standard Problem for Micromagnetic Simulations Including Spin-transfer Torque,"' Journal of Applied Physics 105 (2009), pp. 113914-113921.

[2] M. J. Donahue, "Parallelizing a Micromagnetic Program for Use on Multi-processor Shared Memory 
Computers," IEEE Transactions on Magnetics 45 (2009), pp. 3923-3925.

[3] J. W. Lau, R. D. McMichael, and M. J. Donahue, "Implementation of Two-dimensional Polycrystalline Grains in Object Oriented Micromagnetic Framework," Journal of Research of the National Institute of Standards and Technology 114(Jan 2009), pp. 57-67.

\section{Uncertainty in Virtual Measure- ments from Quantum Chemistry Models}

\author{
Raghu Kacker \\ Rüdiger Kessel \\ Karl Irikura (NIST CSTL) \\ Russell Johnson (NIST CSTL)
}

By a virtual measurement we mean a prediction along with its associated uncertainty for the value of a measurand determined from a computational model as an alternative to a physical measurement. An important application is quantum chemistry, where carefully determined uncertainties have not been reported. As the technology improves, the need and importance of reliable uncertainties in virtual measurements is being recognized. This project is focused on developing and applying methods for quantifying the uncertainty associated with a virtual measurement in quantum chemistry. The benefits accrue to research and development of chemical processes, materials development, and drug discovery.

Predictions from computational quantum chemistry models seldom agree with the corresponding high-quality physical measurements. The differences are not random but systematic. Therefore, a common practice is to apply an empirical scaling factor to computational predictions to bring them closer to the true quantity values. The empirical scaling factor carries uncertainty. We have developed a methodol- ogy to quantify the uncertainty associated with a scaling factor. This approach is based on the Guide to the Expression of Uncertainty in Measurement, which is an international standard. The uncertainties for scaling factors lead to the corresponding uncertainties for virtual predictions. In 2005 we reported uncertainties in the scaling factors for $a b$ initio vibrational frequencies from 40 models (combinations of theory and basis sets) intended for predicting fundamental frequencies from computed harmonic frequencies. The uncertainties were larger than generally acknowledged. Subsequently in 2006-2007, we determined a new reference data set of 60 diatomic and 15 polyatomic experimental vibrational zeropoint energies (ZPEs) that includes estimated uncertainties. In 2008 we refined the methodology for calculating uncertainties and reported uncertainties for 32 models intended for predicting vibrational ZPEs form computed harmonic frequencies. The ZPE paper appeared this year in the Journal of Chemical Physics [1]. An erratum was published to correct an insignificant error in one measurement.

In FY 2009 we determined uncertainties for virtual measurements from computed an-harmonic vibrational frequencies. A paper on this topic has been submitted for publication [2]. Subsequently, some small changes were implemented in the latest version of a research-software (Gaussian) used in our calculations. So we are recalculating to verify our results.

[1] K. Irikura, R. Johnson, R. Kacker, and R. Kessel, "Uncertainties in Scaling Factors for ab-initio Vibrational Zero-Point Energies," Journal of Chemical Physics 130 (11)(2009), pp. 114102-114111.

[2] R. Johnson III, K. Irikura, R. Kacker and R. Kessel, "Scaling factors and uncertainties for ab-initio anharmonic vibrational frequencies," in review. 


\section{Pervasive Information Technology}

Pervasive information technology is the trend towards increasingly ubiquitous connected computing sensors, devices, and networks that monitor and respond transparently to human needs. The Pervasive Information Technologies Program facilitates the creation of standards for sensor communication, networking interoperability, and sensor information security. The Program enables the use of pervasive information technologies to enhance personal and professional productivity and quality of life. - Kamran Sayrafian-Pour, Program Manager

\section{Visualization of Radio Frequency Propagation for Body Area Net- works}

\author{
John Hagedorn \\ Judith Terrill \\ Adele Peskin \\ Kamran Sayrafian-Pour (NIST/ITL) \\ Wen-Bin Yang (NIST/ITL)
}

See feature article, page 35.

\section{Self-organization of Mobile Sensor Networks}

\section{Vladimir Marbukh \\ Kamran Sayrafian-Pour (NIST ITL)}

Mobile sensor networks are envisioned for detecting and tracking potential targets and events for civilian as well as military purposes. Sensor locations in a mobile network affect both the sensor's ability to acquire information on the intended target(s) and event(s) as well as the sensor's ability to communicate this information to the intended recipient(s). Information acquisition needs, which require proximity to the target(s), often compete with communication needs, which require proximity to the recipient(s) of the sensor information. Communication ability can be improved if sensors are capable of forming and optimizing a multi-hop Mobile Ad-hoc NETwork (MANET) with some sensors relaying the other sensors' information possibly in addition to transmitting their own information. The Network Utility Maximization (NUM) framework for crosslayer network optimization has been successfully applied in various networking domains from wire-line network to MANET [1]. NUM optimizes trade-offs between demands of different users in a networked system by a distributed, price-based algorithm.

In [2] we propose NUM extension intended to balance competing mobile sensor network information acquisition and communication goals. Since achieving both these goals depends on the sensor locations relatively to the target(s), to the access point(s), and to each other, the system utility to be maximized is a complex function of sensor rates and locations. The appeal of this unified view of mobile sensor network optimization is that while system utility maximization over sensor information flow rates in "fast" time scale yields the optimal cross-layer design of the sensor MANET, system utility maximization over sensor locations in "slow" time scale guides sensor repositioning. Realization of this vision, however, requires ability to quantify the sensor rate and location dependent system utility. Due to possible redundancy of information acquired by different sensors, the aggregate utility of sensor information streams may not be the sum of utilities of information streams from different sensors.

It is natural to assume that the sensor information recipient, who fuses all sensor information into a coherent picture, is in the best position to estimate the marginal utility of each sensor information stream. On the other hand, each mobile sensor has direct knowledge of its remaining battery power supply and surrounding terrain which affect sensor ability to communicate and relocate. In [3] we propose to resolve this information asymmetry by assuming that mobile sensor network self-organization is guided by the sensor information fusion center. The proposed framework for self-organization of mobile sensor networks includes cross-layer network optimization as well as controlled sensor mobility. Given sensor locations, cross-layer network optimization allocates resources and configures protocols to ensure the delivery of the highest utility of the sensor information to the access point taking into account battery energy conservation needs. This framework overcomes deficiencies of numerous existing phenomenological approaches to sensor mobility control based on phenomenologically defined "potential" fields and corresponding virtual forces, which do not account for the specific sensor information acquisition and communication needs, e.g., different "power costs" of mobile sensors with different remaining battery energy levels.

[1] X. Lin, N. B. Shroff, R. Srikant, "A tutorial on crosslayer optimization in wireless networks," IEEE Journal of Selected Areas of Communications, Vol. 24, Issue 8, pp. 1452-1463, 2006. 
[2] V. Marbukh and K. Sayrafian-Pour, "A Framework for Joint Cross-Layer and Node Location Optimization in Mobile Sensor Networks," in Proceedings of AdhocNow'09, Nice, France, 2008.

[3] V. Marbukh and K. Sayrafian-Pour, "Mobile Sensor Network Self-organization for System Utility
Maximization: Work in Progress," in Proceedings of the International Conference on Wireless and Mobile Communications 2009 (ICWMC'09), Cannes, France, 2009. 


\section{Trustworthy Information Systems}

A trustworthy system is one that performs as intended for a specific purpose, when needed, with operational resiliency and without unwanted side effects, behaviors, or exploitable vulnerabilities. The Trustworthy Information Systems (TIS) Program conducts research, development, and testing to improve the ability to model, build, test, measure, and assess information system trustworthiness through the development and application of new and innovative technologies, models, measurement methods, and tools. The aim of the TIS program is to reduce the risk and uncertainty associated with information systems that must be trusted, and to improve the ability to build in and evaluate trustworthiness in information applications and systems. Tom Rhodes, Program Manager

\section{Automated Combinatorial Testing for Software-systems (ACTS)}

\author{
Raghu Kacker \\ Rüdiger Kessel \\ James Lawrence \\ Stephen Langer \\ Andrew Reid \\ Jeff Yu Lei (U. Texas, Arlington) \\ Rick Kuhn (NIST ITL) \\ Vincent Hu (NIST ITL) \\ Thomas Rhodes (NIST ITL) \\ Renee Bryce (Utah State University) \\ Sreedevi Sampath (U. Maryland Baltimore County) \\ Tao Xie (North Carolina State University)
}

A combinatorial interaction fault is a defect that may cause a complex software system to fail when particular values of certain parameters combine with particular values of other parameters. Combinatorial interaction faults often remain dormant until the unfortunate combination of values is encountered in practice. Many critical system failures in recent times have been traced to interaction faults in the underlying software. Combinatorial testing is an approach to detect such interaction faults. It is a critical technology for ensuring trustworthy software in business, industrial, medical, scientific, and transport systems.

It is often difficult or impossible to characterize all possible combinatorial interactions among software input parameters, let alone test them. Combinatorial testing is based on the observation that most interaction faults involve only a few parameters. Several studies of actual faults show that most involved a single parameter, a smaller proportion resulted from interactions between values of two parameters, progressively fewer interaction faults involved $3,4,5$, and 6 parameters. So far a fault involving more than six parameters has not been seen. Most combinatorial testing has so far been limited to pair-wise (2-way) testing in which all interactions involving two parameters are detected because development of efficient test methods to detect higher order interaction faults have not been available. The goal of this project is to advance the technology from pair-wise to higher order (strength) testing and to demonstrate successful applications.

In 2008 we issued version 1 of a publicly available research tool, FireEye, to generate test suites for high strength testing. The name subsequently was changed to ACTS (Advanced Combinatorial Test Suites) because FireEye was trademarked. In September 2009 we released version 9. Major improvements include the following.

1) Base-Choice Testing allows the user to designate a base value for each parameter (which is typically the default or most frequently used value), and then it combines other values with these base values in a well-defined manner.

2) Test Output Specification allows the user to specify the output parameters (in addition to input parameters) and their expected values for each test case. Expected outcome is needed for test evaluation.

3) New Interface for Constraint Support allows the user to free-type constraints, which is much more user-friendly than button-clicks. The new release also adds constraint support to the command line interface. Significant validation support has also been added in the backend to ensure the legality of constraints.

4) Miscellaneous Improvements have been continually made in response to user feedback. Examples include better error handling, additional export formats, better mixed-strength support, and various GUI enhancements such as in-/out-synchronization (between system configurations and test sets) indicators, new relation/constraint display format in the tree view, and buttons that are made more contextsensitive, and so on.

We have distributed over 265 copies of ACTS tool to US government agencies and global corporations including Microsoft, Lockheed Martin, Cisco, SAP, AT\&T, Intel, and SAIC. The Mid-Atlantic section of the Federal Laboratory Consortium recognized ACTS 
tool with its Regional Excellence in Technology Transfer Award on September 17, 2009.

We have published eight papers in refereed publications and three magazine articles. We have ongoing collaborations with the Food and Drug Administration and the Applied Physics Lab of Johns Hopkins University. We are investigating applications in computer security, health care, GUI based applications, webapplications, and for optimization and testing of simulations of complex systems.

[1] R. Kuhn, R. Kacker, Y. Lei, and J. Hunter, "Combinatorial Software Testing," Computer, August 2009, pp. 9496.

[2] W. Wang, S. Sampath, Y. Lei, and R. Kacker, "An Interaction-based Test Sequence Generation Approach for Testing Web-applications," in Proceedings of 11-th IEEE High Assurance Systems Engineering Symposium, Nanjing, China, December 3-5, 2008.
[3] B. Johnasson, R. Kacker, R. Kessel, C. McLean, and R. Sriram, "Utilizing Combinatorial Testing to Detect Interactions and Optimize a Discrete Event Simulation Model for Sustainable Manufacturing," in Proceedings of the $14^{\text {th }}$ Design for Manufacturing and the Life Cycle Conference, San Diego, CA, August 30-September 2, 2009.

[4] R. Bryce, Y. Lei, D. R. Kuhn, and R. Kacker, "Combinatorial Testing" in Handbook of Research on Software Engineering and Productivity Technologies: Implications of Globalization, to appear.

[5] W. Wang, Y. Lei, S. Sampath, R. Kacker, R. Kuhn and J. Lawrence, "A Combinatorial Approach to Building Navigation Graphs for Dynamic Web Applications," Proceedings of 25th IEEE International Conference on Software Maintenance, to appear.

[6] J. Lawrence, R. Kacker, Y. Lei, D. R. Kuhn, and M. Forbes. "Binary Covering Arrays," in review. 


\section{Division Programs}

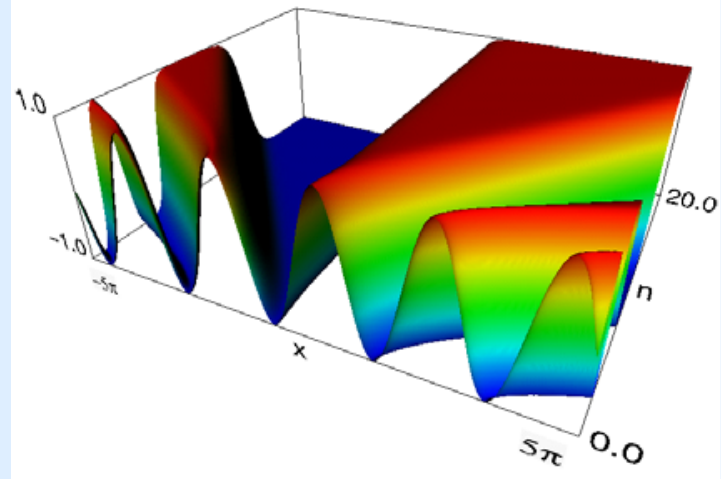

Digital

Library of

Mathematical

Functions

Mathematics of

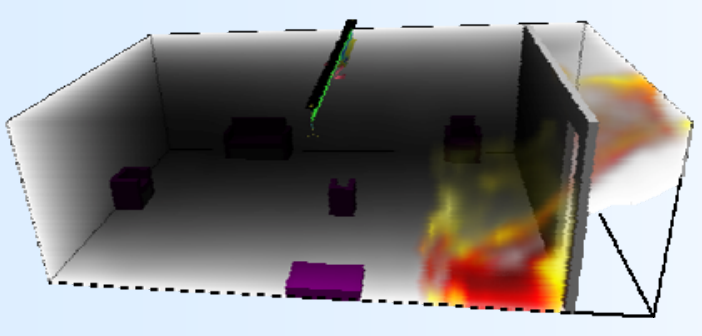

Metrology

High

Performance

Computing and

Visualization

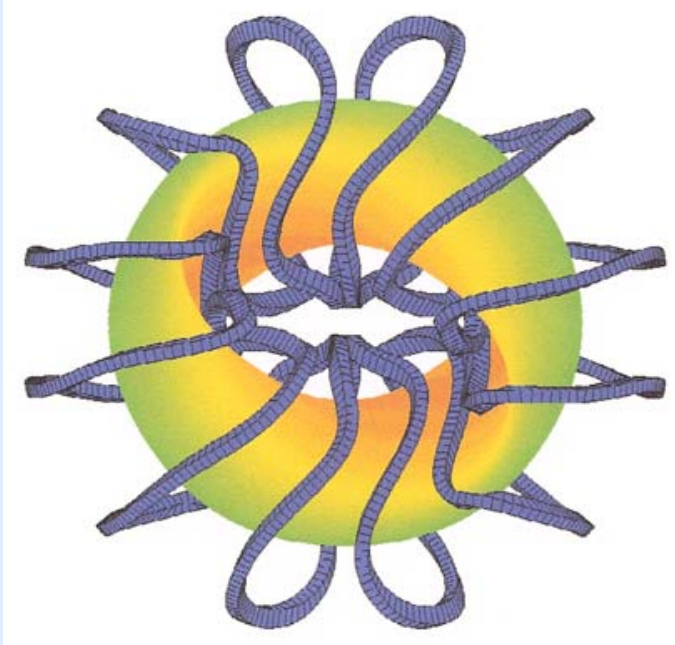

Fundamental Mathematical Software Development 


\section{Digital Library of Mathematical Functions}

The special functions of applied mathematics are fundamental tools enabling modeling and analysis in all areas of science and engineering. To make effective use of such functions, practitioners must have ready access to a reliable source of information on their properties. The goal of this work is the development and dissemination of definitive reference data on the special functions of applied mathematics. The DLMF will be a freely available interactive and richly linked online resource. - Daniel Lozier, Program Coordinator

\section{Digital Library of Mathematical Functions}

\author{
Daniel Lozier \\ Ronald Boisvert \\ Peter Ketcham \\ Marjorie McClain \\ Bruce Miller \\ Frank Olver \\ Bonita Saunders \\ Abdou Youssef \\ Brian Antonishek (NIST ITL) \\ Charles Clark (NIST PL)
}

Eight associate editors, 28 authors, and 23 validators at universities and research institutions

\section{http://dlmf.nist.gov/}

Mathematics and science go hand in hand. Purely mathematical developments find application in the description of scientific phenomena, seemingly by serendipity; in the other direction, cutting-edge science is a major motivator and driver of mathematical research. Often the mathematical objects at the intersection of math and science are mathematical functions. Thus there has always been an interest in, and a practical need for, accurate and comprehensive compilations of the many intricate properties of mathematical functions.

Traditionally, massive published handbooks have been the accepted method of supplying compilations of scientific reference data. An outstanding example for mathematical reference data is the 1,000-page NBS Handbook of Mathematical Functions, created in 1964 under the editorship of Milton Abramowitz and Irene Stegun. The impact of this handbook was tremendous and attracted immediate attention, especially among physical scientists and engineers. It remains a standard reference today, even though it was never updated.

The first goal of the current project is to produce a new handbook that serves the same readership and takes into account the many advances of the last halfcentury in the theory and computation of mathematical functions. The second goal is to develop a novel Web service that takes advantage of, and builds upon, the powerful capabilities of the Web to disseminate essentially the same information as is contained in the new handbook. The new handbook is called the NIST Handbook of Mathematical Functions (HMF), and the Web service is called the NIST Digital Library of Mathematical Functions (DLMF).

After a decade of effort by the project leaders, assisted by NIST personnel and many external experts, the full HMF and DLMF will be publicly available early in 2010. External authors under contract to NIST produced the 36 chapters of the HMF and transferred their copyrights for retention by NIST. These chapters were independently checked by validators, and they were also checked and edited for consistent style and format by the NIST editors (Olver, Lozier, Boisvert, and Clark). Highly qualified NIST experts (Miller, Saunders, and Youssef) led the development of the DLMF, taking advantage of the latest Web technology and making significant research contributions to mathematical representation and semantics, mathematical graphics, and mathematical search.

The HMF is licensed for production, marketing, and distribution by Cambridge University Press, which has already begun its sales campaign; see Figure 31. The DLMF will be a Web service ${ }^{5}$ operated by NIST.

The HMF contains

o Three methodology chapters covering parts of algebra, real and complex analysis, asymptotics, and numerical analysis which are important for work with mathematical functions.

o Thirty-three chapters covering the elementary functions and selected higher, or special, functions.

o Definitions, notations, and properties useful in current or potential applications of mathematical functions in scientific and related fields.

o Graphics, including many line graphs and surface plots for real and complex arguments and parameters.

o Methods of computation and approximation.

o Examples of special functions applications in physics, other scientific and engineering fields, and applied mathematics.

\footnotetext{
${ }^{5}$ http://dlmf.nist.gov/
} 


\section{Cambridge | Catalogue}

Home > Catalogue > NIST Handbook of Mathematical Functions

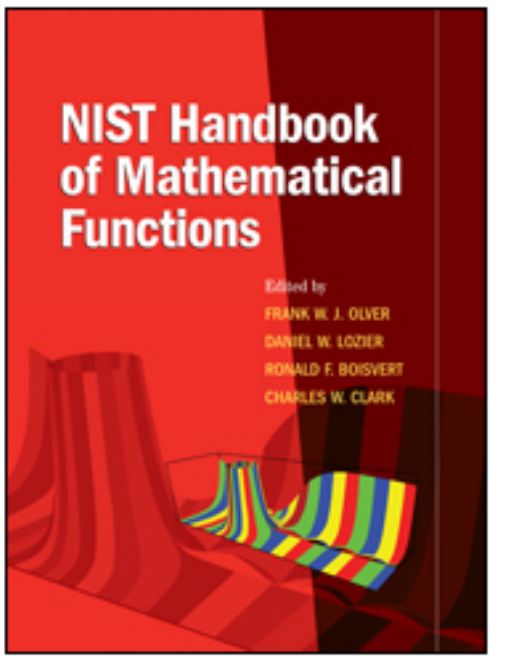

\section{NIST Handbook of Mathematical Functions}

Companion to the Digital Library of Mathematical Functions

Edited by F. Olver

National Institute for Standards \& Technology

D. Lozier

National Institute for Standards \& Technology

R. Boisvert

National Institute for Standards \& Technology

C. Clark

National Institute for Standards \& Technology

View list of contributors...

Figure 31. Announcement of the NIST Handbook in the Cambridge University Press online catalog.

o Large bibliography and index.

The DLMF provides for

o Delivery of the full content of the HMF in a carefully designed Web-oriented format, that contains links to external reviews and full texts of bibliographic items, links to external sources of mathematical software, and links to the HMF definitions of symbols and HMF cross-references.

o Delivery of text and math in XML and MathML to Web browsers that support these technologies, or of text in HTML and math in GIF images to less capable Web browsers, and detection of browser capabilities automatically at the Web server.

o Zoomable and rotatable color visualizations of surfaces of real or complex functions of two real variables in browsers that support VRML or X3D.

o Search based on mathematical queries as well as text queries (for example, the query " $\sin ^{\wedge} 2+$ $\cos ^{\wedge} 2$ " locates many identities that contain the sum of squares of sines and cosines).

Several future developments related to the DLMF project are planned or under consideration. One is to further the semantic capabilities of the XML tools and techniques developed for the DLMF. Another is to develop tools and techniques for generating validated numerical values and graphs on demand. A third is to extend the power and applicability of the DLMF search tools and techniques. Also, resumed development of chapters on Probability Functions and Computer Algebra is planned.

\section{Visualization of Complex Function Data}

\author{
Bonita Saunders \\ Qiming Wang \\ Brian Antonishek (NIST ITL) \\ Sandy Ressler (NIST ITL) \\ Daniel Lozier \\ Frank Olver
}

http://dlmf.nist.gov

Understanding of high level mathematical functions arising from the solution of complex mathematical or physical problems can be greatly enhanced by manipulating accurate visualizations of those functions. Consequently, we have put a substantial effort into finding ways to create precise and informative visualizations of complex function surfaces that can be manipulated inside a web environment. While we originally thought this to be a somewhat minor task, we quickly discovered that our options for creating such visualizations were limited both in number and quality. Using techniques from numerical grid generation and web-based visualization technologies such as the Virtual Reality Modeling Language (VRML) and X3D, we have successfully created accurate visualizations that capture key function features such as zeros, poles, and branch cuts. We have produced over 200 interactive 3D visualizations for the NIST Digital Library of Mathematical Functions (DLMF). 


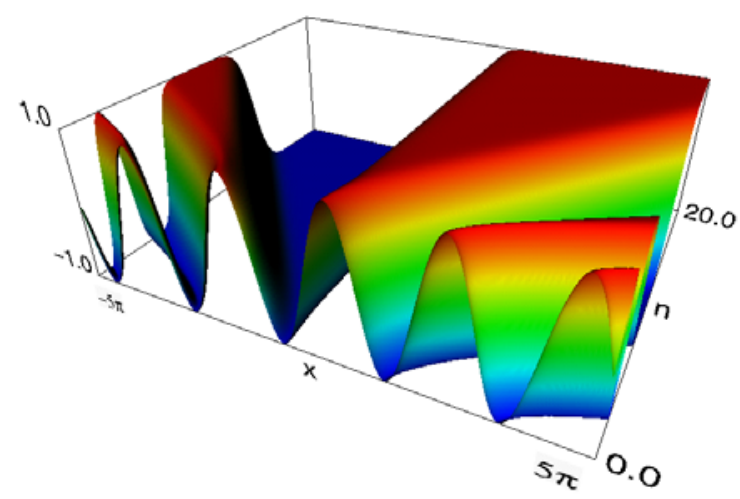

Figure 32. Plot of Jacobian elliptic function $\operatorname{sn}(x, k)$ where $k=$ $1-\exp (-n)$.

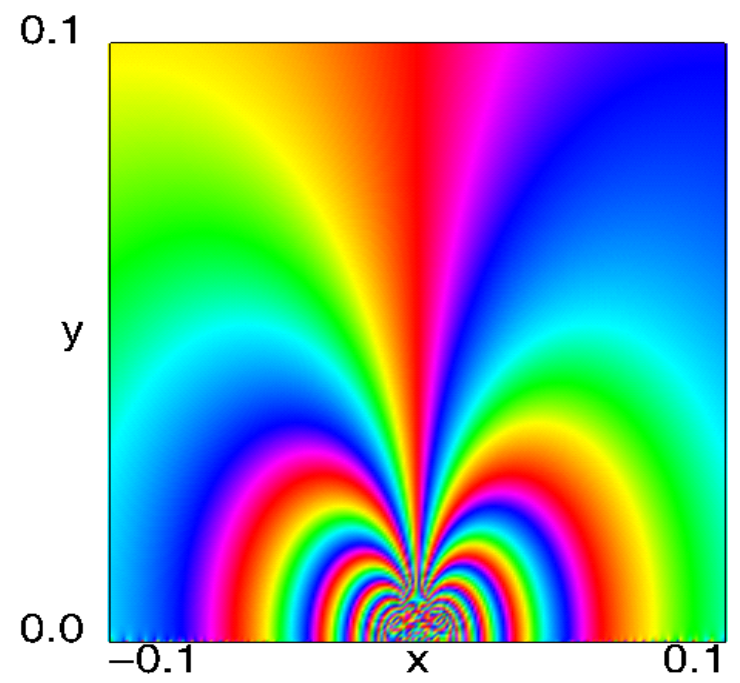

Figure 33. Phase density plot of Dedekind's eta function $\eta(x+i y)$.

Having completed all the visualizations for the first release of the DLMF, our primary focus this year was on improving the presentation quality of the visualizations for the website, validating plot data, and making the visualizations accessible on multiple platforms. The validation of the data has been completed and substantial progress has been made in refining the underlying computational grids in order to improve surface color maps.

Ensuring that our visualizations are accessible on multiple platforms has been one of the more problematic issues. While the DLMF VRML files work well on Windows systems, where most VRML browsers are provided as browser plugins, problems appear in Linux and MacOS where VRML browsers are usually standalone applications. Such applications can be made to operate like plugins by connecting them to the browser as helper applications, but they may not be able to de- tect the location of support routines located outside the main VRML file. Fortunately, we found a way to imbed all the routines and images needed by a particular figure into its main VRML file. This key breakthrough made it possible to view the DLMF VRML files on Mac systems and helped us make substantial progress on Linux systems. We have been able to view a stripped down VRML file, containing only surface geometry information without our customized cutting plane, color map, and scaling features, on a Linux system. We plan to provide this type of file for users whose systems cannot handle the full featured DLMF VRML files.

Also, in record time we successfully converted the DLMF VRML files into X3D (Extensible 3D) using a VRML to X3D translator, with minor post-processing, designed by Qiming Wang several years ago. X3D, which uses an XML type format, is becoming more prevalent for the manipulation and viewing of 3D graphics on the web. Access to these files will also be put on the website shortly.

As our visualization work for the current phase of the NIST DLMF winds down, we will concentrate more on the on-demand generation of mathematical graphs and tables. This may be a stand-alone capability or ultimately be incorporated as part of the DLMF. We are researching websites that offer some of these capabilities and looking at the possibility of extending work on the live generation of tables started by Marjorie McClain, Joyce Conlon and former NIST postdoc and guest worker Bruce Fabijonas.

[1] B. Saunders and Q. Wang, "Tensor Product Grid Generation for Complex Surface Visualizations in a Digital Library of Mathematical Functions," in Proceedings of the $11^{\text {th }}$ International Society for Grid Generation (ISGG) Conference, Montreal, Canada, May 25-28, 2009, p. SV.3.

[2] B. Saunders and Q. Wang, "Tensor Product B-Spline Mesh Generation for Accurate Surface Visualizations in the NIST Digital Library of Mathematical Functions," in Proceedings of the Seventh International Conference on Mathematical Methods for Curves and Surfaces, Tonsberg, Norway, June 26-July 1, 2008, to appear.

[3] Q. Wang, B. Saunders, and S. Ressler, "Dissemination of 3D Visualizations of Complex Function Data for the NIST Digital Library of Mathematical Functions," $\mathrm{CO}$ DATA Data Science Journal 6 (2007) (Supplement on the Proceedings of the $20^{\text {th }}$ International CODATA Conference, Beijing, China, October 2006), pp. S146- S154.

[4] B. Saunders and Q. Wang, "From B-Spline Mesh Generation to Effective Visualizations for the NIST Digital Library of Mathematical Functions," in Curve and Surface Design: Avignon 2006, Sixth International Conference on Curves and Surfaces, Avignon, France, Neshkoro Press, 2007, pp. 235-243. 


\section{Mathematics of Metrology}

Mathematics plays an important role in the science of metrology. Mathematical models are needed to understand how to design effective measurement systems, and to analyze the results they produce. Mathematical techniques are used to develop and analyze idealized models of physical phenomena to be measured, and mathematical algorithms are necessary to find optimal system parameters. Finally, mathematical and statistical techniques are needed to transform the resulting data into useful information. Our goal is to develop fundamental mathematical methods and analytical tools necessary for NIST to continue as a world-class metrology institute, and to apply them critical measurement science applications.

\section{Multihazard Structural Optimiza- tion}

\section{Florian Potra \\ Emil Simiu (NIST BFRL)}

The objective of multihazard structural engineering is to develop methodologies for achieving designs that are safe and cost-effective under multiple hazards. Optimization is a natural tool for achieving such designs. In general, its aim is to determine a vector of design variables subjected to a given set of constraints, such that an objective function of those variables is minimized. In the particular case of structural design, the design variables may be member sizes; the constraints pertain to structural strength and serviceability (e.g., keeping the load-induced stresses and deflections below specified thresholds); and the objective function is the structure's cost or weight. In a multihazard context, the design variables are subjected to the constraints imposed by all the hazards to which the structure is exposed. In particular, the design variables should be determined in such a way that the loadinduced stresses and deflections are kept below specified thresholds at all points of the resulting structure. Since there are infinitely many such points, the optimization problem becomes a semi-infinite programming problem.

In this project, we formulated the multihazard structural design problem in nonlinear programming terms, and presented some simple illustrative examples involving two hazards: earthquake and strong winds. We have also developed efficient bootstrap strategies for solving the semi-infinite programming problem. Our numerical experiments show that interior-point methods are significantly more efficient than classical optimization methods in solving the nonlinear programming problem associated with our illustrative example.

The results concerning the semidefinite programming aspect were presented at the Eleventh International Conference of Optimum Design of Structures and Materials in Engineering (OPTI 2009), Algarwe, Portugal, June 8-10, 2009, and published in the proceedings in that conference [1]. Other results were published in [2] and [3].

[1] F. A. Potra and E. Simiu, "Semi-infinite Programming in Multi-hazard Structural Design," Computer Aided Optimum Design in Engineering XI, in Proceedings of the Eleventh International Conference of Optimum Design of Structures and Materials in Engineering (OPTI 2009), Editors: S. Hernandez and C.A. Brebbia, WIT Press, 2009, pp. 13-19.

[2] F. A. Potra and E. Simiu, "Optimization and Multihazard Structural Design," Journal of Engineering Mechanics 135 (1)2 (2009), pp. 1472-1475.

[3] F. A. Potra and E. Simiu, "Multihazard Design: Structural Optimization Approach," Journal of Optimization Theory and Applications, 2009, Online First, DOI 10.1007/s10957-009-9586-4.

\section{A Mathematical Model of Atmos- pheric Retention of Man-made $\mathrm{CO}_{2}$ Emissions}

\section{Bert Rust}

Climate change skeptics have now mostly conceded that the Earth is indeed warming, but they still strongly deny that the warming is caused by man's activities. In 2008, Rust and Thijsse showed that from 1856 to the present, global annual average temperatures have varied linearly with atmospheric $\mathrm{CO}_{2}$ concentrations. That relationship is somewhat obscured by a 71 year cycle in the temperature data which, though quite visible (see the lower plot in Figure 36), accounts for only about $8 \%$ of the total variance in the measured record. By contrast, the accelerating baseline obtained from the $\mathrm{CO}_{2}$ relationship accounts for about $77 \%$ of that variance.

The present study shows how the increases in atmospheric $\mathrm{CO}_{2}$ concentration can be derived from increases in man-made $\mathrm{CO}_{2}$ emissions, thus completing the connection between man's activities and global warming. This relationship is established without using a complicated global climate model, but rather by using simple mathematical modeling techniques to 
connect and synthesize six different measured data sets containing 5 different kinds of measured quantities.

Figure 34 shows that man-made $\mathrm{CO}_{2}$ emissions to the atmosphere have increased quasi-exponentially since 1850 . The estimated fraction remaining in the atmosphere was calculated by solving a linear inverse problem. Estimates obtained by two different approaches are shown in Figure 35. The forward problem assumed a harmonic expansion for the unknown function, using a basic period of 150 years. The number of harmonics was chosen by optimizing a fit of the emissions remaining in the atmosphere to the record of atmospheric $\mathrm{CO}_{2}$ concentrations shown in the top plot in Figure 36. The inverse problem approach was more difficult because the regression model was highly underdetermined, with an $83 \times 153$ matrix. Estimation was possible only because the solution is constrained to be between 0 and 1 . There is good agreement between the two estimates in spite of the high uncertainties in the first half of the record. The similarity of the two dashed curves in Figure 36 strongly suggests that manmade $\mathrm{CO}_{2}$ emissions are driving the observed global warming.

[1] B. W. Rust, "Atmospheric Retention of Man-made $\mathrm{CO}_{2}$ Emissions," in MAMERN'09: Proceedings of the 3rd International Conference on Approximation Methods and Numerical Modeling in Environment and Natural Resources, B. Amaziane, D. Barrera, M.A. Fortes, et. al., (eds.), Universidad de Granada (June 2009), pp. 813818.

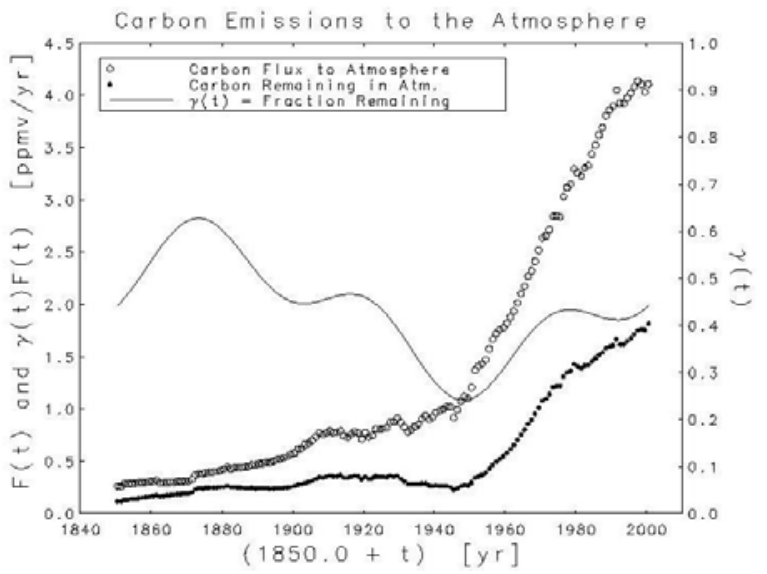

Figure 34. The uppermost plot gives yearly total $\mathrm{CO}_{2}$ emissions to the atmosphere. The smooth curve, scaled on the right, is an estimate of the fraction of those emissions that remain in the atmosphere. The lower plot, the product of the other two, estimates the emissions remaining in the atmosphere.

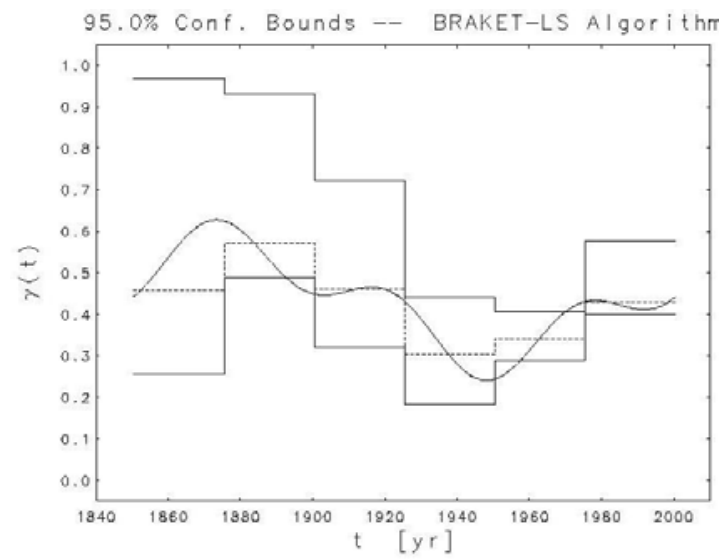

Figure 35. Two estimates of the fraction of $\mathrm{CO}_{2}$ emissions remaining in the atmosphere. The smooth curve is the forward problem estimate, and the dashed piecewise constant curve is an inverse problem estimate for 6 nonoverlapping 25-year averages. The solid piecewise constant curves are 95\% confidence bounds for those averages.

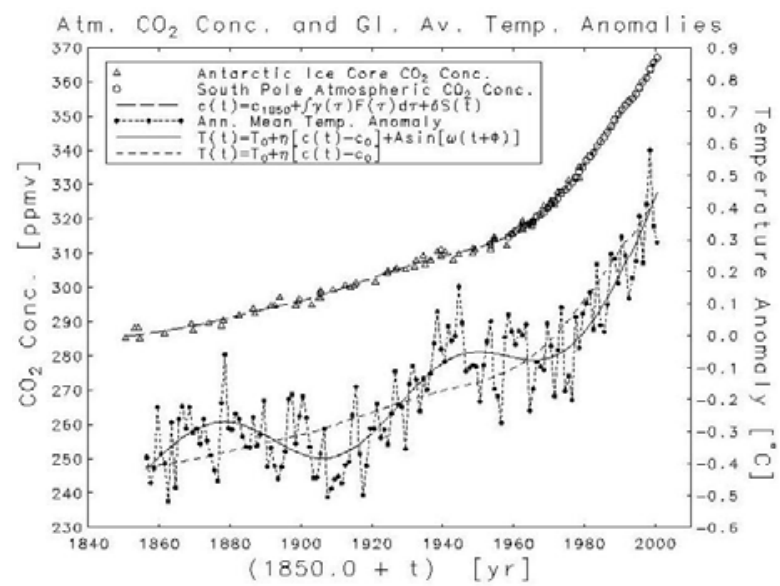

Figure 36. The relationship between atmospheric carbon dioxide and global temperatures. The dashed curve in the upper plot is the estimate of atmospheric concentrations of $\mathrm{CO}_{2}$ given by the retention fraction plotted in Figure 34. The dashed curve in the lower plot is the temperature response to those concentrations. 


\section{Simulation of Bioregulatory Net- works Involved in Cell Cycle Control}

Geoffrey McFadden

S. Kim (National Institutes of Health)

Augustin Luna (NIH and Boston University)

Michael Sharpnack (New York University)

Mirit Aladjem (National Institutes of Health)

Kurt Kohn (National Institutes of Health)

G. McFadden of MCSD is a co-advisor to S. Kim, a postdoctoral fellow, and A. Luna, a graduate student from Boston University, who are both doing research at the Laboratory of Molecular Pharmacology in the National Cancer Institute at $\mathrm{NIH}$; their co-advisors are NIH researchers M. Aladjem and K. Kohn. The team is developing models of bioregulatory networks that are involved in cell cycle control. The models consist of systems of nonlinear ordinary differential equations or delay differential equations that typically exhibit switching behavior, limit cycles, and other types of bifurcations.

Proper cell growth depends on a network of interacting molecules that monitors cellular metabolism and environmental signals. This network ensures that cells halt their growth in response to unfavorable conditions such as the absence of sufficient nutrients or the presence of potentially damaging agents. When cells escape these controls, the results are developmental abnormalities, genomic instability, and cancer. Much current work focuses on a protein known as p53, a tumor suppressor that causes cell cycle arrest or programmed cell death in response to stress signals such as DNA damage. Regulating the appropriate levels of p53 is essential for cell survival. Two associated proteins, Mdm2 and Mdmx, are known regulators of $\mathrm{p} 53: \mathrm{Mdm} 2$ can facilitate degradation of $\mathrm{p} 53$ whereas the mechanism of the regulatory interaction of Mdmx with p53 is not clear. It is also not obvious how those three molecules will operate together under various conditions.

To address those questions a mathematical model has been developed [1] to investigate the interactions of these three partner molecules by numerical simulations. An interesting feature of this system is the experimental observation of time-periodic behavior of the measured amounts of p53 and Mdm2 in the system under certain conditions. These results show the stabilizing effect that Mdmx has on the system at long times: for large enough amounts of Mdmx in the system the entire branch of steady state solutions is found to be linearly stable. This modeling work is intended to guide experimental investigations of the role of Mdmx in the cell cycle that are being performed by Dr. Kim at NIH.

A. Luna has recently joined the team to conduct his $\mathrm{Ph} . \mathrm{D}$. research though a combination of stochastic

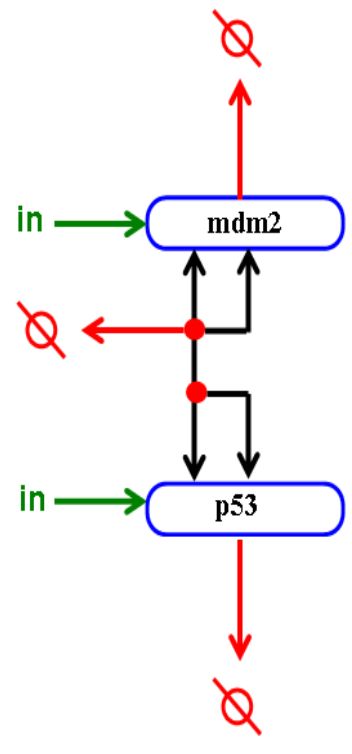

Figure 37. A molecular interaction map showing the reactions between p53 and mdm2 in a simple model. The red dots indicate formation of a complex p53:mdm2, which can degrade to form products indicated by the red null set symbols; the reactants ('in') forming p53 and mdm 2 are also indicated. Each arrow denotes a reaction taking place at a specified rate.

and deterministic modeling of cell cycle regulation. M. Sharpnack, an undergraduate at New York University, was a SURF student in MCSD during the summer of 2009, and worked on a project entitled "A mathematical analysis of the switch-like and oscillatory behaviors of the tumor suppressor p53 feedback loop." An example of one of the simplified networks considered by Mr. Sharpnack in shown in Figure 37.

[1] S. Kim, M. I. Aladjem, G. B. McFadden, and K. W. Kohn, "Predicted Functions of MdmX in Fine Tuning the Response of p53 to DNA Damage," PLoS Computational Biology, in press.

\section{Modeling the Fluid Dynamics of Tear Films on the Cornea}

\author{
Geoffrey B. McFadden \\ Richard J. Braun (University of Delaware) \\ R. Usha (Indian Institute of Technology) \\ P. E. King-Smith (Ohio State University)
}

Dry eye disorders refer to eye problems associated with insufficient amounts of fluids coating the surface of the cornea. They can be caused by reduced production of aqueous tears, and/or increased evaporation due to a deficient lipid layer in the fluid. In both of these conditions, tear film breakup is more rapid than normal. Breakup can be observed either as dark spots in fluorescein-stained tear films, or, noninvasively, by 
distortions in the reflected image of a grid. In a recent study [1] we evaluated the contributions of evaporation and tangential flow to thinning of the pre-corneal tear film between blinks. It was argued that tangential flow of the aqueous tears is largely determined by the movement of the lipid layer. The results indicated that the observed movement of the lipid layer was too slow to explain the observed thinning rate of the tear film, suggesting that most of the observed tear thinning between blinks is due to evaporation.

In order to better understand the dynamics of the spreading of tear films on the human eye we have gone on to develop in more detail two hydrodynamic models based on lubrication theory [2]. The eye is modeled by an ellipsoid of revolution, using a prolate spheroidal coordinate system. One model is a self-consistent leading-order hyperbolic PDE for relatively large substrate curvatures; the other retains another order resulting in a fourth order parabolic PDE for the film dynamics. We consider both Newtonian viscous flow models and nonNewtonian models that take into account shearthinning of the tear fluid. We explore a wide parameter range of shear thinning and find a significant effect on finite-time singularities present in the model, representing discontinuous solutions allowed by the underlying hyperbolic PDE. Approximate results illustrating the nature of the flows are obtained both numerically and analytically. We are able to use this approach to evaluate the influence of the cornea's shape in providing a driving force for fluid motion through the LaplaceYoung boundary condition that relates pressure and interface curvature, an effect that has been neglected in previous studies.

[1] P. E. King-Smith, B. A. Fink, J. J. Nichols, K. K. Nichols, R. J. Braun, and G. B. McFadden, "The Contribution of Lipid Layer Movement to Tear Film Thinning and Breakup," Investigative Ophthalmology \& Visual Science 50 (2009), pp. 2747-2756.

[2] R. J. Braun, R. Usha, G. B. McFadden, T. A. Driscoll, L. P. Cook, and P. E. King-Smith, "Thin Film Dynamics on a Prolate Ellipsoid with Application to the Cornea," submitted to AIMS Journal on Discrete and Continuous Dynamical Systems A (2009).

\section{Modeling Equilibrium, Stability and Transport during Magnetic Fusion}

\section{Geoffrey B. McFadden \\ Paul R. Garabedian (Courant Institute, NYU)}

A hot plasma of hydrogen isotopes can be confined in a strong magnetic field with toroidal geometry so the ions fuse to form helium and release energetic neutrons. Models of such magnetic fusion devices developed in computational science have been imple-

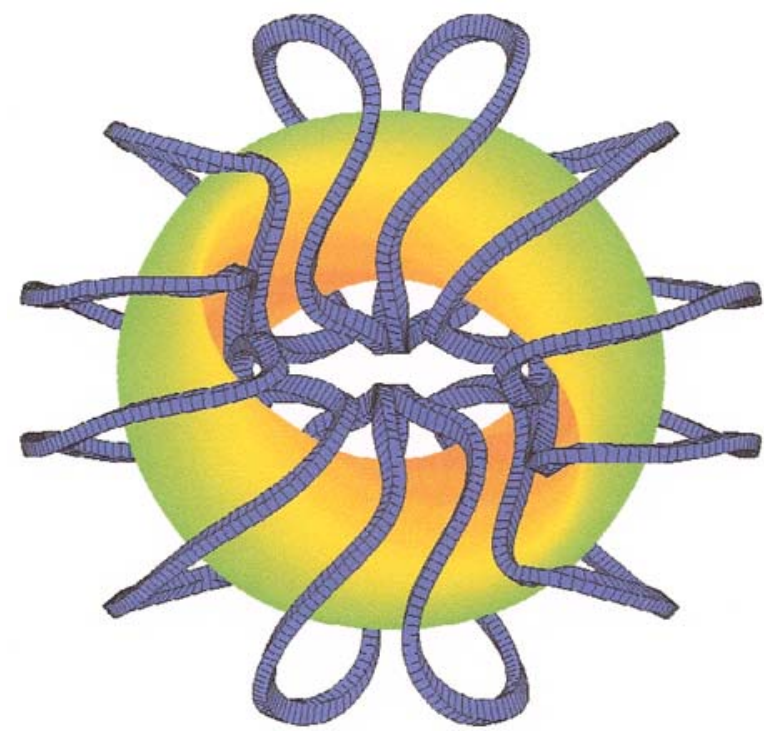

Figure 38. Magnetic fusion of hydrogen forms helium and emits energetic neutrons from a torus of plasma shaped to optimize confinement. Modular coils generate a field keeping the ions from hitting walls.

mented previously by P. Garabedian and his students and colleagues at New York University in codes that comprise an effective numerical simulation of the most essential features of modern tokamak and stellarator experiments. This has led to the discovery of advanced concepts that make fusion reactors a future prospect for a commercial source of energy. In recent work [1] we have applied computer codes [2,3] to issues of equilibrium, stability and transport that provide an example of the theory.

The equilibrium code employs a divergence-free representation of the magnetic field that minimizes a discretization of the energy using a combination of spectral techniques in the toroidal and poloidal directions, and finite differences in the radial direction (outward from the plasma's magnetic axis). The stability of the nonlinear equilibrium states is assessed by applying appropriate perturbations to the system and determining whether they lead to multiple steady states that indicate the occurrence of bifurcated solutions. Transport properties of the charged species are estimated by following the orbits of test particles that track the magnetic field lines between particle collisions. The codes have been used to evaluate possible stellarator designs as candidates for follow-up ("DEMO") experiments that may eventually be performed after the completion of planned international experiments ("ITER") that are based on tokamak configurations.

Tokamaks have axisymmetric symmetry, which is an advantage for purposes of particle confinement, but they rely on strong toroidal currents in the plasma to generate the magnetic fields that can lead to stability issues. The magnetic fields for stellarators are generated by external coils which can alleviate many of the 
stability problems of tokamaks, but generally lack the high symmetry of tokamaks associated with enhanced confinement. The stellarator designs reported recently $[1,4]$ offer the advantages of a type of quasiaxisymmetry (see Figure 38 ) that combines the best features of each, with the possibility of both good stability and transport properties.

[1] P. R. Garabedian and G. B. McFadden, "Design of the DEMO Fusion Reactor following ITER," Journal of Research of the NIST 114 (2009), pp. 229-236.

[2] M. Taylor, "A High Performance Spectral Code for Nonlinear MHD Stability," Journal of Computational Physics 110 (1994), pp. 407-418.

[3] N. Kuhl, "Monte Carlo Simulation of Transport," Journal of Computational Physics 129 (1996), pp. 170-180.

[4] G. B. McFadden, "QAS Design of the DEMO Reactor," $17^{\text {th }}$ International Stellarator/Heliotron Workshop, Princeton Plasma Physics Laboratory, 12-16 October 2009; Communications in Plasma Physics, submitted.

\section{Modeling Type Ia Supernova Light Curves}

\author{
Bert Rust \\ Dianne O'Leary \\ Katharine Mullen (NIST MSEL)
}

The regularity and uniformity of their light curves make Type Ia supernovae the best available candidates for the role of "standard candle" in estimating extragalactic distances. An empirically established correlation between their peak absolute magnitudes and the rates of decline of their light curves provides a means of estimating the peak absolute magnitude of a distant supernova from measurements of its rate of decline in brightness. The estimate thus obtained can then be combined with a measurement of its peak apparent magnitude to give an estimate of its distance. We have developed a mathematical model for the luminosity which, using only six adjustable parameters, gives superior fits to measured light curves and should prove useful in calibrating the correlation described above. An example is given in Figure 39 where the model explains $99.79 \%$ of the variance in the record.

Type Ia supernova luminosity is thought to be powered by the radioactive decay chain $\mathrm{Ni} \rightarrow \mathrm{Co} \rightarrow$ $\mathrm{Fe}$, even though observed luminosity decline rates do not match the terrestrial decay rates of $\mathrm{Ni}$ and $\mathrm{Co}$. In 1976 Rust, Leventhal and McCall [1] presented evidence supporting the hypothesis that those decay rates are accelerated in a supernova because the decays occur in the interior of a carbon-oxygen white dwarf star at extremely high temperatures and densities. Our model [2] revives this idea and uses a three-parameter Weibull probability density function to model the

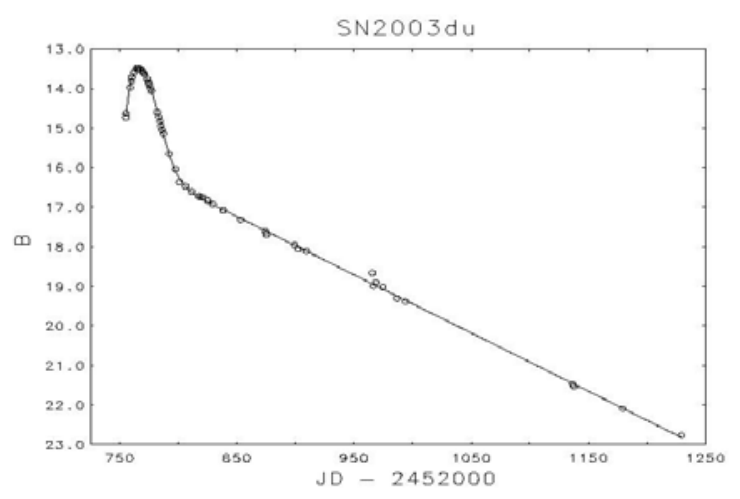

Figure 39. The B-magnitude light curve for the supernova SN2003du. The time variable is measured in Julian Day units. The discrete data points are the observed B-magnitudes and the smooth curve is the model fit.

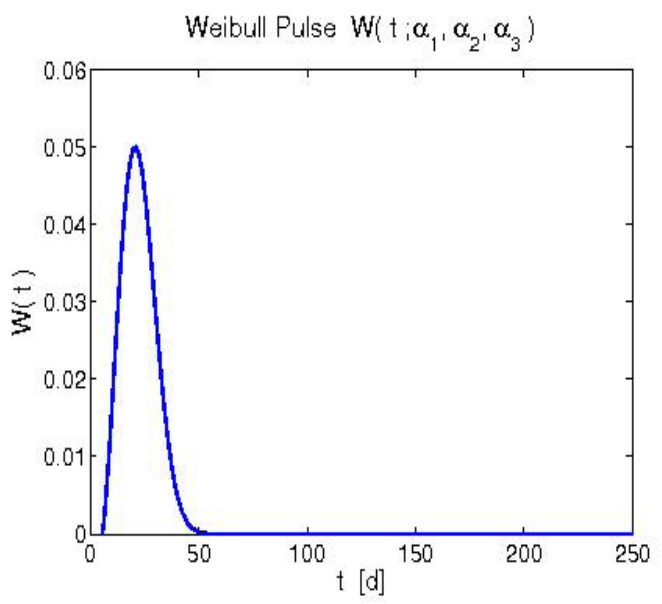

Figure 40. The Weibull pulse powering the Ni deposition in SN1999dq. Here it is normalized to subtend unit area, but it would have to be multiplied by a scaling constant to model the amount of Ni that is actually deposited. The time variable is days since the beginning of the fusion reactions that produced the nickel.

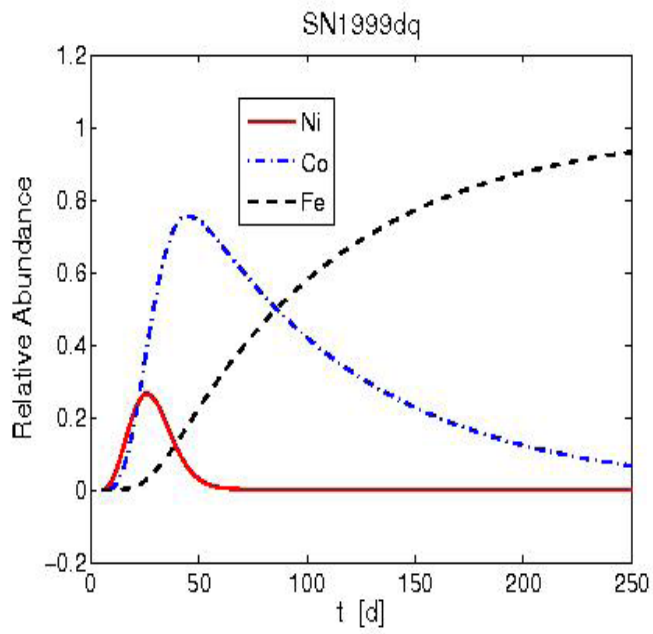

Figure 41. The relative abundances of $\mathrm{Ni}, \mathrm{Co}$, and $\mathrm{Fe}$ during the evolution of the light curve for SN1999dq. The time scale here is the same as in Figure 40. 
deposition of the Ni which is the product of fusion reactions between the carbon, oxygen and lighter elements. An example of this Weibull pulse is plotted in Figure 40.

Plots of the corresponding relative abundances of $\mathrm{Ni}, \mathrm{Co}$, and $\mathrm{Fe}$ are given in Figure 41. These abundances are modeled by a system of three coupled ordinary differential equations involving four adjustable parameters. Three of those parameters specify the Weibull pulse, and the fourth is the common acceleration that must be applied to the terrestrial decay rates of $\mathrm{Ni}$ and Co. Two more adjustable fitting parameters are required to convert the relative abundances to observed luminosities. The final six-parameter model gives much better fits to observed light curves than any previous theoretical model. The previous models have all posited a complete disruption of the star by the supernova explosion, but this model requires the star to remain intact to maintain the high densities needed to produce the stable accelerated decay rate observed in the long tail of the light curve. None of the observed luminosity is produced by the fusion reactions that deposit the Ni.

[1] B. W. Rust, M. Leventhal and S. L. McCall. "Evidence for a radioactive decay hypothesis for supernova luminosity," Nature 262 (1976), pp. 118-120

[2] B. W. Rust, D. P. O'Leary, and K. M. Mullen, "Modeling Type Ia Supernova Light Curves," Exponential Data Fitting and its Applications, Victor Pereyra and Godela Scherer, Eds., to appear. 


\section{High Performance Computing and Visualization}

Computational capability is advancing rapidly. This means that modeling and simulation can be done with greatly increased fidelity (e.g. higher resolution, more complex physics). However, developing large-scale parallel applications remains highly challenging, requiring expertise that application scientists rarely have. In addition, the hardware landscape is changing rapidly so new algorithmic techniques must constantly be developed. We are developing and applying facilities and expertise of this type for application to NIST problems. Large scale computations and laboratory experiments invariably produce large volumes of scientific data, which cannot be readily comprehended without some form of visual analysis. We are developing the infrastructure necessary for advanced visualization of scientific data, including the use of $3 D$ immersive environments and applying this to NIST problems. One of our goals is to develop the 3D immersive environment into a true interactive measurement laboratory.

\section{Visualizing Fire and the Resulting Deformation of Structures}

William Hess

Marc Olano

Judith Terrill

Dilip Banerjee (NIST BFRL)

Researchers in the NIST Building and Fire Research Laboratory (BFRL) are currently studying the effects of fire on building structures to improve structural engineering and building safety. We are using a sequential process in which the BFRL Fire Dynamics Simulator (FDS) is used to simulate the start and propagation of fire in a room, after which a second code calculates how the heat computed by FDS diffuses into the structural members. A third program takes the output of the second code and computes how the structure deforms over time due to combined ef- fects of thermal and mechanical loads. We use a test case with a single beam in a room. We link the fire, thermal, and structural data with a separate real-time visualization capability that can be run on the desktop and in the NIST 3D immersive environment. This enables interactive analysis of the resulting data.

This year we applied volume visualization to the beam in the fire, and created measurement tools for it. We handle all preprocessing of data with a Python script simplifying data and program management. Another Python program reads FDS input files and automatically creates the geometry for the visualization environment. We implemented a Python script for interpolation, simplifying the transformation of output between programs. This script is very general, with the ability to handle any number of datasets relating to beam. We modified the open source physics engine ODE, to get windows to blow out of their frames. We created a multi-channel volume visualization capability and applied it to the fire and smoke. This visualization

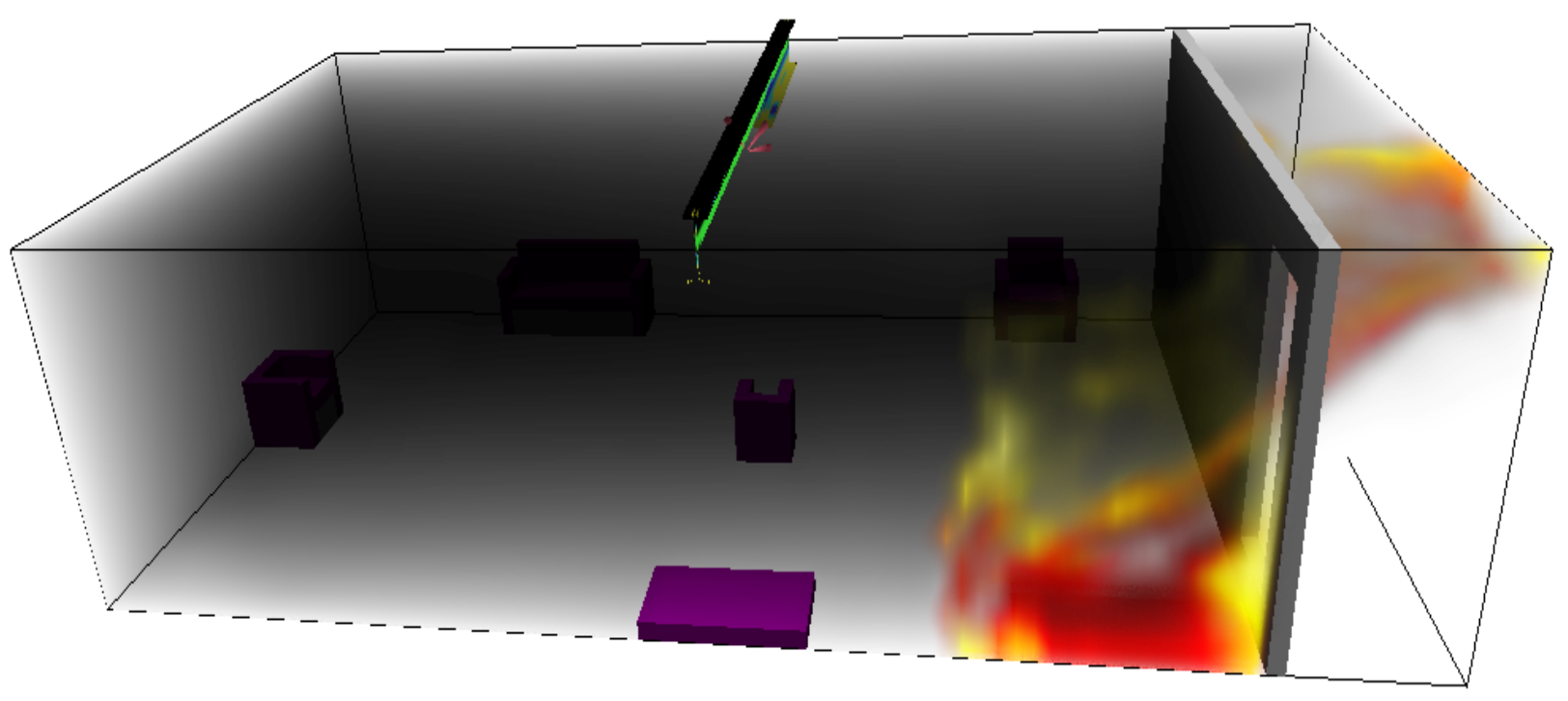

Figure 42. Multichannel volume visualization of fire and smoke. 


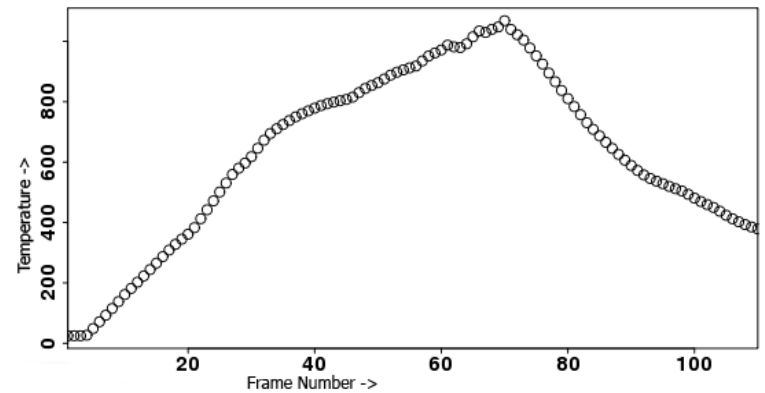

Figure 43. Graph of Temperature Vs Frame number for a selected point on beam

technique draws the bounding box of the volume and any clipping planes using a ray-casting shader. The shader integrates the ray through the volume until it exits the volume through the box, a clipping plane, or by hitting one of the solid objects. The colors are computed using a shader function. The color of the flame is selected from a pseudo-black body spectrum based on the fire temperature. The opacity of the flame comes from the heat release rate per unit volume (a measure of where the burning is actually happening). The smoke is black with an opacity based on the soot density. All three data values are available at each voxel in each time step, and combined to create the final color. See Figure 42. Individual points can be selected on the beam and the graph of temperature vs. time displayed. See Figure 43. We developed a specialty GUI to interact with the data that allows all of the above including cutting planes.

[1] D. Banerjee, W. Hess, M. Olano, J. Terrill, and J. Gross, "Visualization of Structural Behavior under Fire," NISTIR 7619, August 2009. 


\section{Fundamental Math Software Development and Testing}

Modern science and engineering in general, and modern measurement science in particular require a wide variety of software tools for scientific discovery, exploration, and analysis. As scientific inquiry becomes deeper and more specialized, so must the supporting software tools. The goal of this work is to develop critical software tools that support measurement science at NIST, as well as computational science and engineering at large.

\section{SciMark, a Web-based Benchmark for Numerical Computing in Java}

\section{Roldan Pozo \\ Bruce Miller}

\section{http://math.nist.gov/scimark}

The NIST SciMark benchmark continues to be one of the most widely used Java scientific benchmarks and recently became an official component of the industry standard SPECjvm2008. In December 2009, a Google search on "java benchmark" reveals SciMark as the \#1 web site out of 2.6 million results. The SciMark suite consists of computational kernels for FFTs, SOR, Monte Carlo integration, sparse matrix multiply, and dense LU factorization; these comprise a representative set of computational styles commonly found in numerical applications.

SciMark can be run interactively from Web browsers, or can be downloaded and compiled for standalone Java platforms. Full source code is provided in Java and $\mathrm{C}$ programming languages for comparison under different compilers and execution environments. The SciMark result is recorded as a series of megaflop rates for the numerical kernels, as well as an aggregate score for the complete benchmark. The current results database lists results of more than 3,000 submissions from computational platforms ranging from palm devices to high-end servers, representing nearly every operating system and virtual machine environment currently in use, including Solaris, FreeBSD, MacOS, Sun OS, IRIX, OSF1, Linux, OS/2, and Windows 95, 98, 2K, ME, NT, XP, Vista, and Windows 7.

SciMark and its kernel components have become a de facto standard in industry and academia. They have been adopted by the Java Grande Benchmark Forum, and Sun Microsystems has used SciMark 2.0 to demonstrate continued floating-point improvements to their Java Virtual Machine (JVM). SciMark is one of 10 benchmarks included in SPECjvm2008 to represent the wide range of existing Java applications. Other benchmarks included in the suite assess performance in areas such as cryptographic operations, audio decoding, graphics processing, database logic, and XML processing. SPECjvm2008 was released on May 7, 2008 by the System Performance Evaluation Corporation13 (SPEC), a non-profit open industry cooperative which develops benchmarks that ensure that the marketplace has a fair and useful set of metrics to differentiate system performance. For example, results of the well-know SPEC CPU benchmarks (such as SPECint, SPECfp, and SPECrate) are typically cited on specification sheets used in the marketing of computer processors. Members of SPEC include AMD, Dell, HP, IBM, Intel, Microsoft, and Sun.

\section{Division Software and Services}

Staff

MCSD maintains a number of significant services in support of the computational science community at large. This includes the following:

o The Guide to Available Mathematical Software ${ }^{6}$ (GAMS): a cross-index and virtual repository of mathematical software components.

o The Matrix Market ${ }^{7}$ : a database of matrices for use in testing software for linear algebra.

o The Digital Library of Mathematical Functions ${ }^{8}$ : an online handbook of the properties of the special functions of applied mathematics

o SciMark ${ }^{9}$ : an benchmark for assessing the performance of Java Virtual Machines for core numerical computing tasks

o The JavaNumerics Web Page ${ }^{10}$ : a directory of resources for numerical computing in Java

Each such resource requires attention of Division staff to add information, make corrections, and respond to user queries.

In addition, MCSD has developed a wide variety of software which is made available for public download. Among the most popular of these are the following (numbers in parenthesis indicate number of source/executable downloads for calendar 2009):

\footnotetext{
${ }^{6}$ http://gams.nist.gov/

7 http://math.nist.gov/MatrixMarket/

8 http://dlmf.nist.gov/

${ }^{9}$ http://math.nist.gov/scimark/

${ }^{10}$ http://math.nist.gov/javanumerics/
} 
o JAMA: elementary numerical linear algebra in Java (17,067).

o TNT: Template Numerical Toolkit, linear algebra components based on $\mathrm{C}++$ templates $(15,312)$.

o OOMMF: micromagnetic modeling system $(5,200)$. Under current development.

o $L A P A C K++$ : $\mathrm{C}++$ version of the popular package LAPACK for dense linear algebra $(6,307)$.

o SparseLib++: sparse linear algebra in $\mathrm{C}++$ $(4,903)$.

o F90gl: standard Fortran interface to OpenGL graphics $(3,530)$.

o REGEN: cryocooler modeling $(1,932)$.

o Spblas: sparse basic linear algebra subprograms $(1,569)$.

o LaTeXML: LaTeX to XML translator $(1,520)$. o OOF: modeling of materials with complex microstructures $(1,204)$. Under current development.

o PHAML: Parallel Hierarchical Adaptive Multilevel, adaptive multigrid solution of partial differential equations (767). Under current development.

Each such software package requires attention of Division staff to add information, make corrections, and respond to user queries, even when the software is no longer under current development.

MCSD maintains a Web server to enable distribution of its products and services. The server continues to see high usage. During calendar year 2009, the server math.nist.gov satisfied more than $7 \mathrm{M}$ requests for pages, or some 19,000 per day. More than 1.6 Gbytes of data was shipped each day. More than 445,000 distinct hosts were served. There have been more than 217M "hits" on MCSD Web servers since they went online as NIST first web servers in 1994. 


\section{Part IV}

\section{Activity Data}

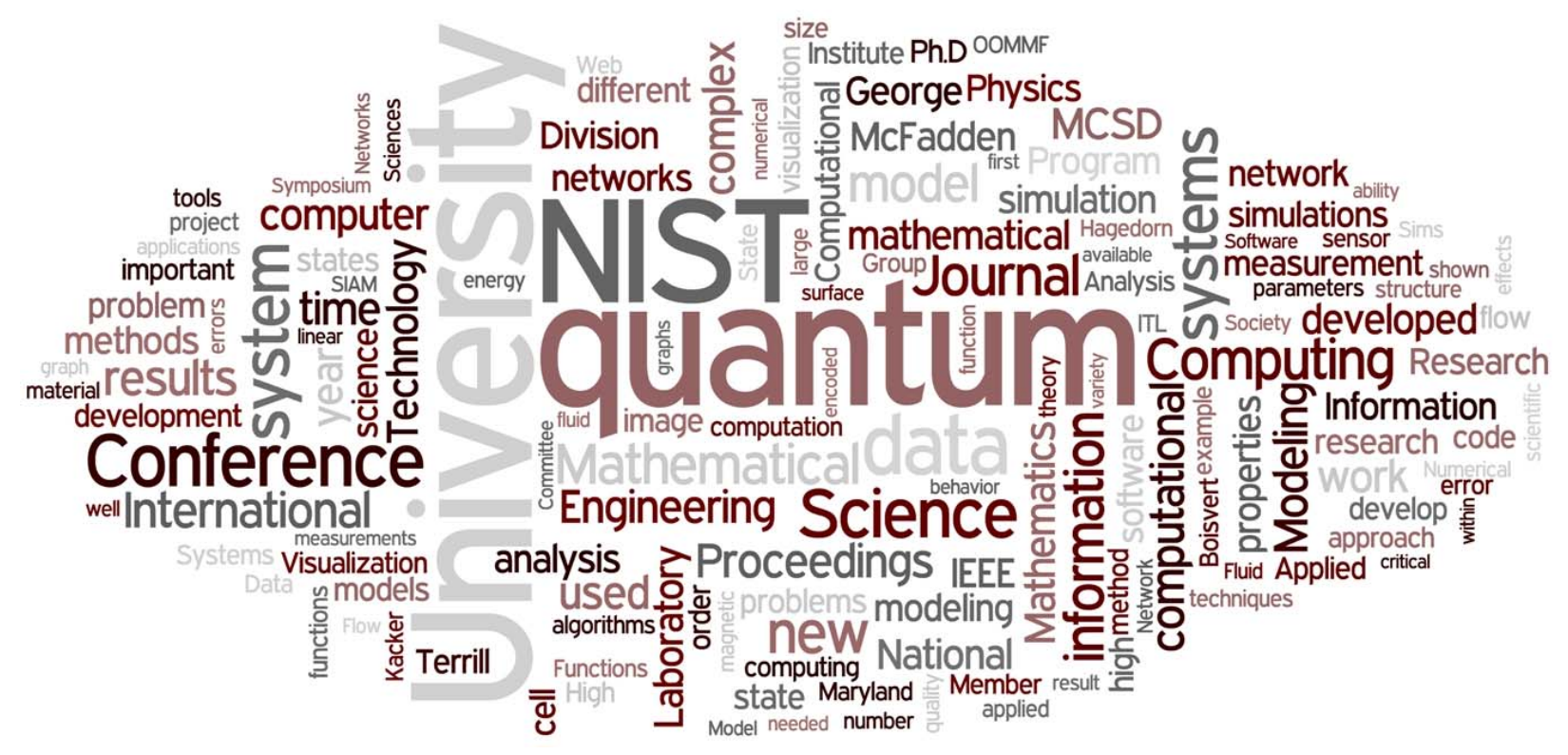




\section{Publications}

\section{Appeared}

\section{Refereed Journals}

1. S. Boixo, E. Knill, and R. D. Somma, "Quantum State Preparation by Phase Randomization," Journal of Quantum Information and Computation 9 (2009), pp. 833-855.

2. S. S. Bullock and D. P. O'Leary, "Locality Bounds on Hamiltonians for Stabilizer Codes," Quantum Information and Computation 9 (2009), pp. 04870499.

3. A. Carasso, "False Characteristic Functions and Other Pathologies in Variational Blind Deconvolution. A Method of Recovery," SIAM Journal of Applied Math 70 (4) (2009), pp. 1097-1119.

4. A. Dienstfrey, T. L. Oreskovic, L. T. Hudson, and H. S. Bennett, "Analysis of ISCD-NIST Survey for Bone Health," Journal of Clinical Densitometry 12 (2) (2009), pp. 162-169.

5. M. J. Donahue, "Parallelizing a Micromagnetic Program for Use on Multi-processor Shared Memory Computers," IEEE Transactions on Magnetics 45 (2009), pp. 3923-3925.

6. B. Eastin and E. Knill, "Restrictions on Transversal Encoded Quantum Gate Sets," Physical Review Letters 102 (11) (2009), article no. 110502 (4 pages).

7. D. E. Gilsinn, "On Algorithms for Estimating Computable Error Bounds for Approximate Periodic Solutions of an Autonomous Delay Differential Equation," Communications in Nonlinear Science and Numerical Simulation 14 (2009), pp. 1526-1550.

8. P. D. Hale, A. Dienstfrey, J. C. M. Wang, D. F. Williams, A. Lewandowski, D. A. Keenan, and T. Clement, "Traceable Waveform Calibration with a Covariance-Based Uncertainty Analysis," IEEE Transactions on Instrumentation and Measurement, 58 (10) (2009), pp. 3554-3568.

9. K. Irikura, R. Johnson, R. Kacker, and R. Kessel, "Uncertainties in Scaling Factors for ab-initio Vibrational Zero-Point Energies," Journal of Chemical Physics 130 (11)(2009), pp. 114102114111.

10. R. Kacker, A. Forbes, R. Kessel, and K.-D. Sommer, "Bayesian Posterior Predictive p-value of Statistical Consistency in Interlaboratory Evaluations," Metrologia 45 (2008), pp. 512-523.
11. R. Kacker and J. Lawrence, "Rectangular Distribution Whose Width is Not Exactly Known: Isocurvilinear Trapezoidal Distribution," Metrologia 46 (2009), pp. 254-260.

12. R. Kessel and R. Kacker, "A Test of Linearity Using Covering Arrays for Evaluating Uncertainty in Measurement," Advanced Mathematical and Computational Tools in Metrology and Testing VIII, Series on Advances in Mathematics for Applied Sciences; 78 (2009), Editors F. Pavese, M. Bdr, A. B. Forbes, J.-M. Linares, C. Perruchet, N. F. Zhang World Scientific, Singapore, pp. 194202.

13. P. E. King-Smith, B. A. Fink, J. J. Nichols, K. K. Nichols, R. J. Braun, and G. B. McFadden, "The Contribution of Lipid Layer Movement to Tear Film Thinning and Breakup," Investigations in Ophthalmology and Visual Science 50 (2009), pp. 2747-2756.

14. R. Kuhn, R. Kacker, Y. Lei, and J. Hunter, "Combinatorial Software Testing," Computer, August 2009, pp. 94-96.

15. Z. H. Levine, M. Li, A. P. Reeves, D. F. Yankelevitz, J. J. Chen, E. L. Siegel, A. Peskin, and D. N. Zeiger, "A Low-Cost Density Reference Phantom for Computed Tomography," Medical Physics 36 (2009), pp. 286-288.

16. N. S. Martys, D. Lootens, W. L. George, and P. Hebraud, "Contact and Stress Anisotropies in Start-up Flow of Colloidal Suspensions," Physical Review E 80 (2009), article no. 031401.

17. G. B. McFadden and S. R. Coriell, "Onset of Oscillatory Convection in Two Liquid Layers with Phase Change," Physics of Fluids 21 (2009), article no. 034101 .

18. Y. Mishin, W. J. Boettinger, J. A. Warren, and G.B. McFadden, "Thermodynamics of Grain Boundary Premelting in Alloys. I. Phase-field Modeling," Acta Materialia 57 (2009), pp. 37713785.

19. M. J. O'Hara and D. P. O'Leary, "The Adiabatic Theorem in the Presence of Noise," Physical Review A 77 (2008), article no. 042319, 20 pages.

20. C. S. Pande, K. P. Cooper, and G. B. McFadden, "Grain Size Distribution in Two Dimensions in the Long Time Limit," Acta Materialia 56 (2008), pp. 5304-5311.

21. A. C. E. Reid, R. C. Lua, R. E. Garcia, V. R. Coffman, and S. A. Langer, "Modeling Microstructures with OOF2," International Journal of Materials and Product Technology 35 (2009), pp. 361-373. 
22. B. W. Rust and D. P. O'Leary, "Residual Periodograms for Choosing Regularization Parameters for Ill-Posed Problems," Inverse Problems 24 (2008), article no. 034005 (30 pages).

23. J. S. Sims and S. A. Hagstrom, "HylleraasConfiguration-Interaction Study of the $2^{2} \mathrm{~S}$ Ground State of Neutral Lithium and the First Five Excited ${ }^{2}$ S States," Physical Review A 80 (2009), article no. 052507 (8 pages).

24. W. C. Uhlig, M .J. Donahue, D. T. Pierce and J. Unguris, "Direct Imaging of Current-driven Domain Walls in Ferromagnetic Nanostripes," Journal of Applied Physics 105 (2009), article no. 103902.

\section{Journal of Research of NIST}

1. P. R. Garabedian and G. B. McFadden, "Design of the DEMO Fusion Reactor Following ITER," Journal of Research of the National Institute of Standards and Technology 114 (2009), pp. 229236.

2. J. W. Lau, R. D. McMichael, M. J. Donahue, "Implementation of Two-Dimensional Polycrystalline Grains in Object Oriented Micromagnetic Framework," Journal of Research of the National Institute of Standards and Technology 114 (2009), pp. 57-67.

\section{Books}

1. B. Balachandran, T. Kalmar-Nagy, D. E. Gilsinn (Ed.), Delay Differential Equations: Recent Advances and New Directions, Springer, New York, 2009.

2. D. P. O'Leary, "Scientific Computing with Case Studies," SIAM Press, Philadelphia, 2009.

\section{Book Chapters}

1. D. E. Gilsinn, "Bifurcations, Center Manifolds, and Periodic Solutions," in Delay Differential Equations: Recent Advances and New Directions, Balakumar Balachandran, Tamas Kalmar-Nagy and David E. Gilsinn (Ed.), Springer, New York, 2009, pp. 155-202.

2. J. Terrill, W. George, T. Griffin, J. Hagedorn, J. Kelso, M. Olano, A. Peskin, S. Satterfield, J. Sims, J. Bullard, J. Dunkers, N. Martys, A. O'Gallagher, and G. Haemer "Extending Measurement Science to Interactive Visualization Environments," in Trends in Interactive Visualization: A-State-of-the-Art Survey, pp. 287-302
(Elena Zudilova-Seinstra, Tony Adriaansen and Robert van Liere, Eds.), Springer, UK.

\section{Conference Proceedings}

1. I. Beichl and B. Cloteaux, "Measuring The Effectiveness Of The s-Metric To Produce Better Network Models," in Proceedings of the Winter Simulation Conference (2008), Miami, FL, December 7-10, 2008, pp. 1020-1028.

2. G. W. Bryant, N. Malkova, J. Sims, M. Zielinski, W. Jaskolski, and J. Aizpurua, "Re-engineering the Optics of Quantum Dots with Mechanical Strain," in Proceedings of Optics of Excitons in Confined Systems (OECS11), Madrid, Spain, Sept. 7-11, 2009.

3. T. J. Burns, S. P. Mates, R. L. Rhorer, E. P. Whitenton, D. Basak, "Dynamic Properties for Modeling and Simulation of Machining: An Update of Results from the NIST Pulse-Heated Kolsky Bar," in Proceedings of the 12th CIRP Conference on Modeling of Machining Operations, San Sebastian, Spain, May 7-8, 2009, pp. 255-262.

4. B. Cloteaux and D. Ranjan, "Lower Bounds for Accessing Information on Pure Pointer Machines," in Proceedings of the International Conference on the Foundations of Computer Science, Las Vegas, NV, July 13-16, 2009, pp. 103-107.

5. C. Dabrowski and F. Hunt, "Using Markov Chain Analysis to Study Dynamic Behavior in LargeScale Grid Systems," in Proceedings of the Seventh Australasian Symposium on Grid Computing and e-Research (AusGrid 2009), CRPIT, 99, Wellington, New Zealand, Jan. 20-23, 2009, pp. 29-40.

6. J. T. Fong, R. deWit, P. V. Marcal, J. J. Filliben, and N. A. Heckert, "A Design-of-Experiments Plug-In for Estimating Uncertainties in Finite Element Simulations," in Proceedings of 2009 International SIMULIA Conference, London, U.K., May 18-21, 2009, pp. 828-842.

7. J. T. Fong, and P. V. Marcal, "Artificial Intelligence Tools for Failure Event Data Management and Probabilistic Risk Analysis for Failure Prevention," in Proceedings of the Materials Science \& Technology 2009 Conference, Pittsburgh, PA, October 25-29, 2009, pp. 529-540.

8. F. Hunt and V. Marbukh, "Measuring the Utility/Path Diversity Trade-Off in Multipath Protocols," in Proceedings of the $4^{\text {th }}$ International Conference on Performance Evaluation, Methods and Tools (ValueTools09), Pisa, Italy, October 2022, 2009 . 
9. V. Marbukh and D. Genin, "Towards Understanding Metastability in Large-scale Loss Network with Mobile Users: Emergence and Implications for Performance," in Proceedings of the IEEE Globecom 2008 Conference, New Orleans, Nov. 30 - Dec. 4 , 2008.

10. V. Marbukh, "From Network Microeconomics to Network Infrastructure Emergence," in Proceedings of the First IEEE International Workshop on Network Science for Communication Networks (NetSciCom'09), Rio de Janeiro, Brazil, April 24, 2009.

11. V. Marbukh and K. Sayrafian-Pour, "A Framework for Joint Cross-Layer and Node Location Optimization in Mobile Sensor Networks," in Proceedings of Adhoc-Now'09, Nice, France, 2008.

12. V. Marbukh and K. Sayrafian-Pour, "Mobile Sensor Network Self-organization for System Utility Maximization: Work in Progress," in Proceedings of the International Conference on Wireless and Mobile Communications, Cannes, France, 2009.

13. P. V. Marcal, J. T. Fong, and N. Yamagata, "Artificial Intelligence (AI) Tools for Data Acquisition and Probability Risk Analysis of Nuclear Piping Failure Databases," in Proceedings of the International Symposium on PVP Inservice Inspection and Failure Event Database," Prague, Czech Republic July 26-30, 2009.

14. N. S. Martys, W. L. George, S. G. Satterfield, D. Lootens, P. Hebraud, and J. Hagedorn, "A Smoothed-particle-hydrodynamics-based Fluid Model with a Local Shear-rate Dependent Viscosity: Application to Flow of a Suspension with a Non-Newtonian Fluid Matrix," in Proceedings of the 2009 Society of Rheology Meeting, Madison, WI, October 18-22, 2009.

15. N. S. Martys, D. Lootens, W. L. George, and P. Hebraud, "Contact and Stress Anisotropies in the Start-up Flow of Colloidal Suspensions," in Proceedings of the 2009 Society of Rheology Meeting, Madison, WI, October 18-22, 2009.

16. W. F. Mitchell, "Strategies for hp-Adaptive Refinement," in Proceedings of the 2008 International Conference on Numerical Analysis and Applied Mathematics, AIP Conference Proceedings 1048, Kos, Greece, Sept. 16-20, 2008, pp. 23-25.

17. A. Peskin, K. Kafadar, A. M. Santos, and G. Haemer, "Robust Volume Calculations of Tumors of Various Sizes," in Proceedings of the 2009 International Conference on Image Processing, Computer Vision, and Pattern Recognition, Las Vegas, NV, July 13-16, 2009.
18. A. Peskin, K. Kafadar, A. Dima, J. Bernal, and D. Gilsinn, "Synthetic Lung Tumor Data Sets for Comparison of Volumetric Algorithms," in Proceedings of the 2009 International Conference on Image Processing, Las Vegas, NV, July 13-16, 2009.

19. A. Peskin, K. Kafadar, and A. Dima, "A Quality Pre-Processor for Biological Cell Images," Computational Bioimaging 2009, 5th International Symposium on Visual Computing, Las Vegas, NV, November 30-December 2, 2009.

20. B. W. Rust, “Atmospheric Retention of Man-made $\mathrm{CO}_{2}$ Emissions," in MAMERN'09: Proceedings of the 3rd International Conference on Approximation Methods and Numerical Modeling in Environment and Natural Resources, B. Amaziane, D. Barrera, M.A. Fortes, et. al., (eds.), Universidad de Granada (June 2009), pp. 813-818.

21. B. Saunders and Q. Wang, "Tensor Product Grid Generation for Complex Surface Visualizations in a Digital Library of Mathematical Functions," in Proceedings of the 11th International Society for Grid Generation (ISGG) Conference, Montreal, Canada, May 25-28, 2009.

22. K. Sayrafian, W. Yang, J. Hagedorn, J. Terrill, and K. Y. Yazdandoost, "A Statistical Path Loss Model for Implant Communication Channels," in Proceedings of the IEEE 2009 Personal, Indoor, and Mobile Radio Communications Symposium, Tokyo, Japan, Sept. 13-16, 2009.

23. X. Tang, L. Ma, A. Mink, T. Chang, H. Xu, O. Slattery, A. Nakassis, B. Hershman, D. Su, and R. F. Boisvert, "High-Speed Quantum Key Distribution System for Optical Fiber Networks in Campus and Metro Areas," in Quantum Communications and Quantum Imaging VI (R. E. Meyers, Y. Shih, and K. S. Deacon, eds.), Proceedings of the SPIE 7092, pp. 70920I-1 - 70920I-15 (2008).

24. H. M. Vasconcelos, L. Sanz, and S. Glancy, "Generation of GKP States with Optical States," in Proceedings of the XXXII Brazilian Meeting on Condensed Matter Physics, Aguas de Lindoia, Brazil, May 11 - 15, 2009.

25. W. Wang, S. Sampath, Y. Lei, and R. Kacker, “An Interaction-based Test Sequence Generation Approach for Testing Web-applications," in Proceedings of 11-th IEEE High Assurance Systems Engineering Symposium, Nanjing, China, December 3-5, 2008.

26. W. Yang, K. Sayrafian-Pour, J. Hagedorn, J. Terrill, "Simulation Study of Body Surface RF Propagation for UWB Wearable Medical Sensors," in Proceedings of the IEEE International Sympo- 
sium on Applied Sciences in Biomedical and Communication Technologies, November 2009.

\section{Other Formal Publications}

1. I. Beichl, "Begin at the Beginning," Computing in Science \& Engineering 11 (1) (2009), p. 3.

2. I. Beichl, "We'll Take Care of All Your Problems," Computing in Science \& Engineering 11 (2) (2009), pp. 3-4.

3. I. Beichl, "Where the Rubber Meets the Road," Computing in Science and Engineering 11 (5) (2009), p 3.

4. I. Beichl and F. Sullivan, "Cut It Out," Computing in Science and Engineering 11 (3) (2009), pp. 7479.

5. W. George, "What Insights Are Revealed When Modeling Concrete's Rheological Properties?," Argonne Leadership Computing Facility Brochure (2008), p. 32.

6. W. George, "Modeling the Rheological Properties of Concrete," in Transforming the World through Breakthrough Science, Department of Energy, Argonne Leadership Computing Facility Annual Report (2008), p. 21.

7. W. George, "Modeling the Rheological Properties of Concrete," in High End Computing at NASA Highlights of Science and Engineering Achievements on NASA's Supercomputers 2007-2008, pp. 112-113.

\section{Technical Reports}

1. D. Banergee, W. Hess, M. Olano, and J. Terrill, "Visualization of Structural Behavior under Fire," NISTIR 7619, August 2009.

2. C. Dabrowski and F. Hunt, "Markov Chain Analysis for Large Scale Grid Systems" NISTIR 7566, April 2009.

3. D. E. Gilsinn, B. R. Borchardt, and A. Tebbe, "Estimating Volumes of Simulated Lung Cancer Nodules," NISTIR 7571, July 2009.

4. C. Witzgall and M. Franaszek, "Sensitivities and Variances for Fitted Parameters of Spheres," NISTIR 7616, July 2009.

\section{Visualizations Published}

1. W. George, N. Martys, S. Satterfield, J. Hagedorn, M. Olano, and J. Terrill, "Simulation of NonNewtonian Suspensions: Shear Thinning Case," movie presented at the 2009 SciDAC Conference
Visualization Night., San Diego, CA, June 14-18, 2009.

2. W. George, N. Martys, S. Satterfield, J. Hagedorn, M. Olano, and J. Terrill, "Simulation of NonNewtonian Suspensions: Shear Thinning Case ${ }^{11}$," movie, in Hadley Leggett, "Best Science Visualizations Videos of $2009^{12}$," Wired Science, on-line magazine, August 19, 2009.

\section{Accepted}

1. I. Beichl and B. Cloteaux, "A Structural Approach to the Temporal Modeling of Networks," in Proceedings of the 2009 Winter Simulation Conference.

2. I. Beichl and B. Cloteaux, "An Approximation Algorithm for the Coefficients of the Reliability Polynomial," Congressus Numerantium.

3. W. J. Boettinger and G. B. McFadden, "Bending of a Bimetallic Beam Due to the Kirkendall effect," Journal of Phase Equilibria and Diffusion.

4. R. Bryce, Y. Lei, D. R. Kuhn, and R. Kacker, "Combinatorial Testing" in Handbook of Research on Software Engineering and Productivity Technologies: Implications of Globalization.

5. J. W. Bullard, E. Enjolras, W. L. George, S. G. Satterfield, and J.E. Terrill, "Validating a Scalable Parallel Computer Model of Cement Hydration and Microstructure Development," Modeling and Simulation in Materials Science and Engineering.

6. J. W. Bullard, C. F. Ferraris, E. J. Garboczi, N. S. Martys, P. E. Stutzman, and J. E. Terrill, "Virtual Cement and Concrete," in Innovations in Portland Cement Manufacturing.

7. R. Datla, R. Kessel, A. Smith, R. Kacker, and D. Pollock "Uncertainty Analysis of Remote Sensing Optical Sensor Data: Guiding Principles to Achieve Metrological Consistency," International Journal of Remote Sensing.

8. J. T. Fong, R. deWit, P. V. Marcal, J. J. Filliben, N. A. Heckert, and S. R. Gosselin, "Design of a Python-based Plug-In for Benchmarking Probabilistic Fracture Mechanics Computer Codes with Failure Event Data," Proceedings of the ASME Pressure Vessels \& Piping Conference.

9. D. E. Gilsinn, B. Borchardt, and A. Tebbe, "Volume Estimation of Molded Artifacts by B-

\footnotetext{
${ }^{11}$ http://www.wired.com/video/simulation-of-nonnewtoniansuspensions/32972797001

${ }^{12} \mathrm{http}: / /$ www.wired.com/wiredscience/2009/08/visualizations/6/
} 
Splines," SIAM Conference on Mathematics for Industry.

10. J. A. Gordon, C. L. Holloway, and A. Dienstfrey, "A Physical Explanation of Angle-Independent Reflection and Transmission Properties of Metafilms/Metasurfaces," IEEE Transactions on Antennas and Propagation.

11. C. L. Holloway, A. Dienstfrey, E. F. Kuester, J. F. O'Hara, A. E. Azad, and A. J. Taylor, "A Discussion on the Interpretation and Characterization of Metafilms/Metasurfaces: The Two-dimensional Equivalent of Metamaterials," Metamaterials.

12. B. Johnasson, R. Kacker, R. Kessel, C. McLean, and R. Sriram, "Utilizing Combinatorial Testing to Detect Interactions and Optimize a Discrete Event Simulation Model for Sustainable Manufacturing," in Proceedings of the $14^{\text {th }}$ Design for Manufacturing and the Life Cycle Conference, San Diego, CA, August 30-September 2, 2009.

13. R. N. Kacker, R. Kessel, K.-D. Sommer, and X. Bian, "Comparison of Statistical Consistency and Metrological Consistency," Proceedings of the $19^{\text {th }}$ World Congress on Fundamental and Applied Metrology.

14. R. Kessel and R. N. Kacker, "Correlation in Uncertainty of Measurement - A Discussion of State of the Art Techniques," in Proceedings of the $19^{\text {th }}$ World Congress on Fundamental and Applied Metrology.

15. S. Kim, M. I. Aladjem, G. B. McFadden, and K. W. Kohn, "Predicted Functions of MdmX in Fine Tuning the Response of p53 to DNA Damage," PLoS Computational Biology.

16. P. A. Lott and H. Elman, "Fast Iterative Solver for Convection-Diffusion Systems with Spectral Elements," Numerical Methods in Partial Differential Equations.

17. V. Marbukh and K. Sayrafian, "Mobile Sensor Networks Self-organization for System Utility Maximization: Work in Progress," The Fifth International Conference on Wireless and Mobile Communications (ICWMC'09).

18. V. Marbukh and D. Genin, "Bursty Fluid Approximation of TCP for Modeling Internet Congestion at the Flow Level," in Forty-Seventh (2009) Annual Allerton Conference on Communication, Control, and Computing.

19. W. F. Mitchell, "The $h p$-Multigrid Method Applied to hp-Adaptive Refinement of Triangular Grids," Numerical Linear Algebra with Applications.
20. J. Obrzut, A. Anopchenko, J. F. Douglas and B. W. Rust, "Relaxation and Antiplasticization Measurements in Trehalose Glycerol Mixtures -A Model for Protein Preservation," Journal of Non-Crystalline Solids.

21. R. Radebaugh, Y. Huang, A. O'Gallagher, and J. Gary, "Optimization Calculations for a $30 \mathrm{HZ}, 4 \mathrm{~K}$ Regenerator with Helium-3 Working Fluid," in Proceedings of the Cryogenic Engineering Conference, Tucson, AZ, June 28 - July 2, 2009.

22. B. W. Rust, D. P. O'Leary, and K. M. Mullen, "Modeling Type Ia Supernova Light Curves," Exponential Data Fitting and its Applications, Victor Pereyra and Godela Scherer, Eds.

23. B. Saunders and Q. Wang, "Tensor Product BSpline Mesh Generation for Accurate Surface Visualization in the NIST Digital Library of Mathematical Functions," Proceedings of the Seventh International Conference on Mathematical Methods for Curves and Surfaces, Tonsberg, Norway, June 26-July 1, 2008.

24. J. S. Sims, G. W. Bryant, W. L. George, T. G. Griffin, J. G. Hagedorn, J. T. Kelso, T. M. Olano, A. P. Peskin, S. G. Satterfield, and J. D. Terrill, "Computation and Visualization of Nanostructures and Nano-optics," Computing and Visualization in Science.

25. W. Wang, Y. Lei, S. Sampath, R. Kacker, R. Kuhn and J. Lawrence, "A Combinatorial Approach to Building Navigation Graphs for Dynamic Web Applications," Proceedings of 25th IEEE International Conference on Software Maintenance.

\section{In Review}

1. D. M. Anderson, G. B. McFadden, S. R. Coriell, and B.T. Murray, "The Solidification of an Ideal Ternary Alloy in a Mushy Layer."

2. R. Braun, R. Usha, G. McFadden, T. Driscoll, L. P. Cook and P. King-Smith, "Thin Film Dynamics on a Prolate Ellipsoid with Application to the Cornea."

3. T. Burns, S. Mates, R. Rhorer, E. Whitenton, and D. Basak, Dynamic Properties for Modeling and Simulation of Machining: Effect of Pearlite to Austenite Phase Transition on Flow Stress in AISI 1075 Steel."

4. A. Carasso, "Bochner Subordination, Logarithmic Diffusion Equations, and Blind Deconvolution of Hubble Space Telescope Imagery and Other Scientific Data." 
5. B. Cloteaux, "Approximating the Number of Bases for Almost All Matroids."

6. D. E. Gilsinn, B. R. Borchardt, and A. Tebbe, "Volume Estimation of Molded Artifacts by BSplines."

7. R. Kessel, R. Kacker, and K.-D. Sommer, "Metrological Consistency and Consensus Result of Multiple Evaluations."

8. E. Knill, "Quantum Computing Q\&A."

9. J. Lawrence, R. Kacker, Y. Lei, D. R. Kuhn, and M. Forbes. "Binary Covering Arrays."

10. H. Mahboubi, K. Moezzi, A. G. Aghdam, K. Sayrafian-Pour, and V. Marbukh, "Distributed Deployment Algorithms for Field Coverage in Mobile Sensor Networks."

11. H. Mahboubi, K. Moezzi, A. G. Aghdam, K. Sayrafian-Pour, and V. Marbukh, "Self-Deployment Algorithms in Coverage Problem for a Network of Mobile Sensors with Unidentical Sensing Range."

12. H. Mahboubi, A. Momeni, A. G. Aghdam, K. Sayrafian-Pour, and V. Marbukh, "Optimal Target Tracking Strategy with Controlled Node Mobility in Mobile Sensor Networks."

13. N. S. Martys, W. L. George, S. G. Satterfield, and M. Olano, "Spatial-temporal Correlations in Startup Flows of Colloidal Suspensions."

14. N. S. Martys, W. L. George, B.-W. Chun, and D. Lootens, "A Smoothed Particle Hydrodynamics Based Fluid Model with a Spatially Dependent Viscosity: Application to Flow of a Suspension with a Non-Newtonian Fluid Matrix."

15. G. B. McFadden, S. R. Coriell, and P. A. Lott, "Onset of Convection in Binary Liquid Layers."

16. G. B. McFadden and P. R. Garabedian, "Quasiaxisymmetric Design of the DEMO Reactor."

17. W. F. Mitchell and M. A. McClain, "A Survey of $h p$-Adaptive Strategies for Elliptic Partial Differential Equations."

18. C. S. Pande and G. B. McFadden, "Self-similar Grain Size Distribution in Three Dimensions."

19. Y. Zhang, E. Knill, and S. Glancy, "The Statistical Strength of Experiments to Reject Local Realism with Photon Pairs and Inefficient Detectors."

\section{$\underline{\text { Presentations }}$}

\section{Invited Talks}

1. R. Boisvert, "Succeeding in a Federal Lab," Future Practitioner Colloquium, INFORMS Annual Meeting, Washington, DC, October 11, 2008.

2. R. Boisvert, "A Handbook of Mathematical Functions for the Digital Age," Department of Computer Engineering, University of Palermo, Italy, July 17, 2009.

3. R. Boisvert, "A Handbook of Mathematical Functions for the Digital Age," International Federation of Information Processing Working Group 2.5 Meeting, Raleigh, NC, August 29, 2009.

4. G. W. Bryant, N. Malkova, J. Sims, M. Zielinski, W. Jaskolski, and J. Aizpurua, "Reengineering the Optics of Quantum Dots," Condensed Matter and Surface Science Seminar, Ohio University, Athens, OH, April 2009.

5. G. W. Bryant, N. Malkova, J. Sims, M. Zielinski, W. Jaskolski, and J. Aizpurua, "Reengineering the Optics of Quantum Dots," Atomic Physics Division Seminar, NIST, May 2009.

6. G. W. Bryant, N. Malkova, J. Sims, M. Zielinski, W. Jaskolski, and J. Aizpurua, "Reengineering the Optics of Quantum Dots with Mechanical Strain," Electronic Materials Conference, Penn State University, University Park, PA, June 2009.

7. B. Cloteaux, "Measurements on Networks," Computer Science Colloquium, New Mexico State University, Las Cruces, NM, April 15, 2009.

8. A. Dienstfrey, "NIST and Virtual Measurement Systems," International Federation of Information Processing Working Group 2.5, Raleigh, NC, August 31, 2009.

9. J. Fong, "Engineers in the Global Economy: Innovative and Symphonic," Mechanical Engineering Department Graduate Course Seminar on Nonlinear Mechanics, Stanford University, Stanford, CA, May 15, 2009.

10. J. Fong, "Engineers in the Global Economy: Innovative and Symphonic," Mechanical Engineering Department Undergraduate Course Seminar on Statics, Stanford University, Stanford, CA, May $15,2009$.

11. J. Fong, "An Uncertainty Estimation Plug-in for Applications in Computational Engineering and Science Modeling," Mathematics and Scientific Computing Group Seminar, National Physical 
Laboratory, Teddington, England, UK, May 20, 2009.

12. J. Fong, "Engineers in the Global Economy: Innovative and Symphonic," Mechanical Engineering Department Seminar, University of South Carolina, Columbia, SC, May 29, 2009.

13. J. Fong, "Engineers in the Global Economy: Innovative and Symphonic," Mechanical Engineering and Mechanics Department Seminar, Drexel University, Philadelphia, PA, June 5, 2009.

14. J. Fong, "Engineers in the Global Economy: Innovative and Symphonic," Civil and Environmental Engineering Department Seminar, University of British Columbia, Vancouver, BC, Canada, June 16, 2009.

15. J. Fong, "Aging Plant Risk-Informed In-Service Inspection (RI-ISI) Methods and Artificial Intelligence Tools for Probabilistic Risk Assessment," Applied Physics Division Seminar, Pacific Northwest National Laboratory, Richland, WA, Sept. $18,2009$.

16. S. Glancy, "Use and Creation of Superpositions of Optical Coherent States," JILA Atomic Molecular Optical Physics Seminar, University of Colorado, Boulder, CO, November 12, 2008.

17. S. Glancy, "Innovations in Maximum Likelihood Quantum State Tomography," Perimeter Institute Quantum Information Seminar, Perimeter Institute, Waterloo, Canada, Sept. 9, 2009.

18. F. Hunt, "The Dynamics of a Model of a Computer Protocol," Mathematical Problems in Industry Workshop 2009, University of Delaware, Newark, DE, June 15-19, 2009.

19. J. Kelso, "DIVERSE and OpenSceneGraph," Virginia Tech, Blacksburg, VA, October 28, 2008.

20. E. Knill, "How Are Your Qubits?" Time and Frequency Division Seminar, NIST Boulder, Feb. 26, 2009.

21. S. Langer, "OOF: Analyzing Material Microstructure," SIAM Conference on Computational Science and Engineering, Costa Mesa, CA, Feb 19-23, 2007.

22. W. F. Mitchell, “ $h p$-Adaptive Finite Elements for the Schrödinger Equation," University of Nevada, Reno, NV, April 24, 2009.

23. D. P. O'Leary, “A Noisy Adiabatic Theorem and Implications for Quantum Computing," AMS Joint Math Meetings, Washington, DC, January 2009.
24. D. P. O'Leary "Validating Approximate Solutions to Ill-Posed Problems," George Mason University, January 30, 2009.

25. D. P. O'Leary “Optimization Methods for Image Deblurring," NASA Goddard Space Flight Center IS\&T Colloquium, Greenbelt, MD, April, 2009.

26. D. P. O’Leary "Validating Approximate Solutions to Ill-Posed Problems," Washington-Baltimore Section of the Society for Industrial and Applied Mathematics Meeting, College Park, Maryland, May 2009.

27. R. Radebaugh, Y. Huang, A. O’Gallagher, and J. Gary, "Optimization Calculations for a $30 \mathrm{HZ}, 4 \mathrm{~K}$ Regenerator with Helium-3 Working Fluid," The Cryogenic Engineering Conference, Tucson, AZ, June 28 - July 2, 2009.

\section{Conference Presentations}

1. B. Alpert, "Compressive Sampling Reconstruction by Subspace Refinement," Institute for Mathematics and Its Applications, University of Minnesota, Workshop on Multi-Manifold Data Modeling and Applications, Minneapolis, MN, October 27-30, 2008.

2. G. W. Bryant, N. Malkova, J. Sims, M. Zielinski, W. Jaskolski and J. Aizpurua, "Re-engineering the Optics of Quantum Dots with Mechanical Strain," Electronic Materials Conference, University Park, PA, June 24-26, 2009.

3. G. W. Bryant, N. Malkova, J. Sims, M. Zielinski, W. Jaskolski, and J. Aizpurua, "Re-engineering the Optics of Quantum Dots with Mechanical Strain," Optics of Excitons in Confined Systems (OECS11), Madrid, Spain, Sept. 7-11, 2009.

4. T. J. Burns, "Dynamic Properties for Modeling and Simulation of Machining: An Update of Results from the NIST Pulse-Heated Kolsky Bar," 12th CIRP Conference on Modeling of Machining Operations, San Sebastian, Spain, May 7-8, 2009.

5. B. Cloteaux, "Measuring The Effectiveness of the s-Metric to Produce Better Network Models," Winter Simulation Conference, Miami, FL, December 9, 2008.

6. B. Cloteaux, "An Approximation Algorithm For The Coefficients Of The Reliability Polynomial," Southeastern International Conference on Combinatorics, Graph Theory, and Computing, Boca Raton, FL, March 5, 2009.

7. V. R. Coffman, "Validating Virtual Polycrystals with OOF," 10th U.S. National Congress on Com- 
putational Mechanics, Columbus, OH, July 17, 2009.

8. M. J. Donahue, "Parallelizing a Micromagnetic Program for Use on Multi-processor Shared Memory Computers," Intermag 2009, Sacramento, CA, May 5, 2009.

9. M. J. Donahue and K. M. Lebecki, "Quasi-stable Vortex Magnetization Structures in Nanowires with Perpendicular Anisotropy," $7^{\text {th }}$ International Symposium on Hysteresis Modeling and Micromagnetics (HMM 2009), Gaithersburg, MD, May 13, 2009.

10. B. Eastin, "Restrictions on Transversal Encoded Quantum Gate Sets," Quantum Information Processing (QIP) Conference, Santa Fe, NM, January 14, 2009.

11. B. Eastin, "Restrictions on Transversal Encoded Quantum Gate Sets," Southwest Quantum Information and Technology (SQuInT) Conference, Seattle, WA, February 20, 2009.

12. J. Fong, "A Design-of-Experiments Plug-In for Estimating Uncertainties in Finite Element Simulations," 2009 Simulia Customers Conference, London, UK, May 18, 2009.

13. J. Fong, “A Dataplot-Python-Anlap (DPA) Plug-in for High Temperature Mechanical Property Databases to Facilitate Stochastic Modeling of FireStructure Interactions," ASME 2009 Pressure Vessels and Piping Division Conference, Prague, The Czech Republic, July 27, 2009.

14. J. Fong, "A Web-based Uncertainty Plug-in (WUPI) for Fatigue Life Prediction based on NDE Data and Fracture Mechanics Analysis," ASME 2009 Pressure Vessels and Piping Division Conference, Prague, The Czech Republic, July 27, 2009

15. J. Fong, "Artificial Intelligence (AI) Tools for Data Acquisition and Probability Risk Analysis of Nuclear Piping Failure Databases," ASME 2009 Pressure Vessels and Piping Division Conference, Prague, The Czech Republic, July 30, 2009

16. J. Fong, "Design of a Python-based Plug-in for Benchmarking Probabilistic Fracture Mechanics Computer Codes with Failure Event Data," ASME 2009 Pressure Vessels and Piping Division Conference, Prague, The Czech Republic, July 30, 2009

17. W. George, S. Satterfield, M. Olano, and J. Terrill, "Flow of Suspensions," movie at the Argonne National Laboratory booth, Supercomputing 2008, Austin, TX, November 15-21, 2008.
18. J. Hagedorn and S. Satterfield, "An Immersive Visualization Environment to Study RF Propagation from Medical Implants," DIVERSE BOF, SIGGRAPH 2009, New Orleans, LA, August 3-7, 2009.

19. J. Kelso, "Introduction to DIVERSE," DIVERSE Birds of a Feather, SIGGRAPH 2009, New Orleans, LA, Aug. 3-7, 2009.

20. P. A. Lott, "Computing Steady Flow States using Fast Iterative Solvers," 5th Annual Symposium of the Burgers Program for Fluid Dynamics, College Park, MD, November 14, 2008.

21. P. A. Lott, "Computing Steady Flow States using Fast Iterative Solvers," 16th Annual Sigma Xi poster session, NIST, Gaithersburg, MD, Feb. 11, 2009.

22. P. A. Lott, "Fast Solver for Models of Fluid Flow," MD-DC-VA MAA Section Meeting, Fredericksburg, VA, April 18, 2009.

23. V. Marbukh, "From Network Microeconomics to Network Infrastructure Emergence," First IEEE International Workshop on Network Science for Communication Networks (NetSciCom'09), Rio de Janeiro, Brazil, April 24, 2009.

24. V. Marbukh and K. Sayrafian, "Mobile Sensor Networks Self-organization for System Utility Maximization: Work in Progress," Fifth International Conference on Wireless and Mobile Communications. (ICWMC 2009), Cannes/La Bocca, France, August 23-29, 2009.

25. N. S. Martys, W. George, S. Satterfield, D. Lootens, P. Hebraud, and J. Hagedorn, "A Smoothed-particle-hydrodynamics-based Fluid Model with a Local Shear-rate Dependent Viscosity: Application to Flow of a Suspension with a Non-Newtonian Fluid Matrix," Society for Rheology 81st Annual Meeting, Madison, WI, October 18-22, 2009.

26. N.S. Martys, D. Lootens, W.L. George, and P. Hebraud, "Contact and Stress Anisotropies in the Start-up Flow of Colloidal Suspensions," 2009 Society of Rheology Meeting, Madison, WI, October 18-22, 2009.

27. N. S. Martys, W.L. George, M. Olano, J. Kelso, J. Hagedorn, S.G. Satterfield, and J. Terrill, "VCCTL Rheology Update," VCCTL Bi-Annual Meeting, Gaithersburg, MD, December 3-4, 2008. .

28. W. F. Mitchell, " $h p$-Adaptive Finite Elements in a Parallel PDE Solver," SIAM Conference on Computational Science and Engineering, Miami, FL, March 2-6, 2009. 
29. W F. Mitchell, "The hp-Multigrid Method Applied to $h p$-Adaptive Finite Elements," Fourteenth Copper Mountain Conference on Multigrid Methods, Copper Mountain, CO, March 22-27, 2009.

30. M. Olano, "Multi-Channel Volume Visualization of Fire and Smoke," DIVERSE BOF, SIGGRAPH 2009, New Orleans, LA, Aug. 3-7, 2009.

31. A. Peskin, K. Kafadar, A. M. Santos, and G. Haemer, "Robust Volume Calculations of Tumors of Various Sizes," The 2009 International Conference on Image Processing, Computer Vision, and Pattern Recognition, Las Vegas, NV, July 13-16, 2009.

32. A. Peskin, K. Kafadar, A. Dima, J. Bernal, and D. Gilsinn, "Synthetic Lung Tumor Data Sets for Comparison of Volumetric Algorithms," The 2009 International Conference on Image Processing, Las Vegas, NV, July 13-16, 2009.

33. D. Porter and M. Donahue, "Exploiting Effective Field Time Derivative Information to Improve Accuracy of a Norm-preserving Landau-Lifshitz Solver," MMM2008, Austin, TX, November 14, 2008.

34. D. Porter (panelist), "Open Discussion with the Tcl/Tk Core Team," Fifteenth Annual Tcl/Tk Conference, Manassas, VA, October 23, 2008.

35. D. Porter and M. Donahue, "Semi-analytical LLG Solver Using Effective Field Time Derivative," $7^{\text {th }}$ International Symposium on Hysteresis Modeling and Micromagnetics (HMM 2009), Gaithersburg, Maryland, May 12, 2009.

36. B. Rust, "Atmospheric Retention of Man-made $\mathrm{CO}_{2}$ Emissions," 3rd International Conference on Approximation Methods and Numerical Modeling in Environment and Natural Resources, Pau, France, June 8-11, 2009.

37. B. Rust, "Using Confidence Ellipsoids to Choose the Regularization Parameter for Ill-Posed Problems," SIAM Conference on Applied Linear Algebra, Monterey Bay - Seaside, California, October 26-29, 2009.

38. B. Saunders, "Tensor Product Grid Generation for Complex Surface Visualizations in a Digital Library of Mathematical Functions," 11th International Society for Grid Generation (ISGG) Conference, Montreal, Canada, May 25-28, 2009.

39. K. Sayrafian, W. Yang, J. Hagedorn, J. Terrill, K. and Y. Yazdandoost, "A Statistical Path Loss Model for Implant Communication Channels," IEEE 2009 Personal, Indoor, and Mobile Radio
Communications Symposium, Tokyo, Japan, Sept 13-16, 2009.

40. J. Terrill, "Opportunities at NIST," Supercomputing 2008 Birds-of-a-Feather: Opportunities in Federal NITRD High End Computing Interagency Working Group (HEC-IWG) Agencies, Austin, TX, November 15-21, 2008.

\section{Patents}

1. Alfred S. Carasso, Singular Integral Image Deblurring Method, Patent Number 7,437,012, October 14, 2008.

\section{Software Released}

1. DiVisa ${ }^{13}$ : A multi-dimensional visualization tool developed for researchers to understand the behavior of their data. Version 8.10.30 - C. Houard and J. Hagedorn.

2. $f 90 \mathrm{gl}^{14}$ : Fortran 90 interface for the OpenGL graphics library. Version 1.2.14 (June 9, 2009). W. Mitchell.

3. NGraph $+{ }^{15}$ : a simplified $\mathrm{C}++$ graph library for analysis of complex networks. Version 1.0.1. $-R$. Pozo.

4. OOF ${ }^{16}$ : Object Oriented Finite Elements, modeling of materials with complex microstructures. Versions 2.0.2 (Feb. 16, 2007), 2.0.5a7 (Dec. 1, 2008), 2.0.5a8 (Dec. 16, 2008), and 2.0.5a9 (Jan. 26, 2009). - S. Langer.

5. Pcrawler ${ }^{17}$ : a Python-based web crawler which generates a web graph of hyperlinks, together with time stamps, SHA checksums, and annotated links so that validation of graph can be independently performed. Version $0.9-R$. Pozo.

6. PHAML ${ }^{18}$ : Parallel Hierarchical Adaptive Multi Level, general purpose software for 2D elliptic partial differential equations. Versions 1.5.0 (October 9, 2008) and 1.6.0 (August 2009). - W. Mitchell

7. Rendering Error Metrics ${ }^{19}$ : Methods for assessing geometric errors introduced by the rendering of three 3D geometric primitive forms: points, line

\footnotetext{
13 http://math.nist.gov/mcsd/savg/software/divisa/

$14 \mathrm{http} / / /$ math.nist.gov/f90gl

15 http://math.nist.gov/pozo/ngraph

${ }^{16} \mathrm{http}: / /$ www.ctcms.nist.gov/oof/

${ }^{17}$ http://math.nist.gov/ RPozo/complex_datasets.html

${ }_{18} \mathrm{http}: / /$ math.nist.gov/phaml/

${ }_{19}$ http://math.nist.gov/mcsd/savg/vis/metrology/index.html
} 
segments, and triangles. - C. Houard, J. Hagedorn, J. Terrill, A. Peskin, and J. Filliben.

8. Sparse BLAS ${ }^{20}$ : with added testing functionality for upper and lower triangular sparse matrices. Version v. 1.0.2-R. Pozo.

9. SparseLib $++^{21}$ : a $\mathrm{C}++$ library for sparse matrix computations, which included updating various modules to the latest ANSI/ISO C++ coding standards, and improved compatibility with multiplatform $\mathrm{C}++$ compilers. Version 1.7 ( ). $-R$. Pozo.

10. Tcl/Tk ${ }^{22}$ : Tool Command Language and Toolkit. Versions 8.6a3 (October 10, 2008), 8.5.5 (October 15, 2008), 8.6b1 (December 23, 2008), 8.5.6 (December 23, 2008), and 8.5.7 (April 15, 2009). - D. Porter.

\section{Conferences, Minisymposia, Lecture Series, Shortcourses}

\section{MCSD Seminar Series}

1. O. Marques (Lawrence Berkeley National Laboratory), "Eigensolvers for Large Electronic Structure Calculations," December 16, 2008.

2. J. M. Burke (Boehringer-Ingelheim Pharmaceuticals), "Mathematical and Experimental Single-cell Analysis of Caspase Amplification in the Death Receptor Network," December 23, 2008.

3. A. Patra (University of Buffalo), "Parallelism and Adaptivity - A Marriage Not Made in Heaven," February 17, 2009.

4. G. Dogan, "Fast Methods to Find Optimal Shapes in Images," April 21, 2009.

5. S. McMaster (University of Maryland), "Advances in Coverage-Based Test Suite Reduction," April 24, 2009.

6. R. Barnard (Louisiana State University), "Modeling Background Noise for Denoising in Chemical Spectroscopy," June 30, 2009.

7. J. Harlim (New York University), "Mathematical Strategies for Filtering Turbulent Systems: Sparse Observations, Model Errors, and Stochastic Parameter Estimation,” July 1, 2009.

8. R. C. Peralta (NIST/ITL), "Small Circuits for Linear Functions," August 24, 2009.

\footnotetext{
${ }^{20} \mathrm{http}: / /$ math.nist.gov/spblas

${ }^{21}$ http://math.nist.gov/sparselib

${ }^{22}$ http://tcl.sourceforge.net/
}

9. S. G. Nash (George Mason University), "Multilevel Optimization Methods for Engineering Design and PDE-Constrained Optimization," September 8, 2009.

10. J. Chung (University of Maryland), "Numerical Methods for Large-Scale Ill-Posed Inverse Problems," September 15, 2009.

\section{Local Events Organized}

1. M. Donahue, Member, Local Organizing Committee, Hysteresis and Micromagnetic Modeling Symposium, Gaithersburg, MD, May 11-14, 2009.

2. M. Donahue, Member, Program Committee, Hysteresis and Micromagnetic Modeling Symposium, Gaithersburg, MD, May 11-14, 2009.

3. D. Porter, Member, Local Organizing Committee, Hysteresis and Micromagnetic Modeling Symposium, Gaithersburg, MD, May 11-14, 2009.

\section{External Events Organization}

1. R. Boisvert, Member, Program Committee, PSE Workshop08: Innovative and Collaborative Problem Solving Environments in Distributed Resources, $4^{\text {th }}$ IEEE International Conference on e-Science, Indianapolis, December 7-12, 2008.

2. R. Boisvert, Co-Organizer, IFIP Working Group 2.5 Symposium on Advances in Numerical Computing and Problem Solving, Raleigh, NC, August 31 - September 1, 2009.

3. M. Donahue, Member, Organizing Committee for MS10, the 6th SIAM meeting on Mathematical Aspects of Materials Science.

4. J. Fong, Minisymposium Organizer, "Materials Property Databases," ASME Pressure Vessel and Piping Conference, Prague, Czech Republic, July 26-30, 2009.

5. J. Fong, Minisymposium Organizer, "PVP Inservice Inspection (ISI) and Failure Event Databases. (NDE)," ASME Pressure Vessel and Piping Conference, Prague, Czech Republic, July 26-30, 2009.

6. J. Fong, Minisymposium Organizer, "Field \& Laboratory Non-destructive Evaluation (NDE) of Residual Stresses," ASME Pressure Vessel and Piping Conference, Prague, Czech Republic, July 26-30, 2009.

7. J. Fong, Minisymposium Organizer, "Reliability of Continuous NDE Monitoring and Uncertainty Estimation of Defect Detection, Location, and 
Sizing," ASME Pressure Vessel and Piping Conference, Prague, Czech Republic, July 26-30, 2009 .

8. F. Hunt, Member, Program Planning Committee, Mathematics Association of America.

9. J. Kelso, Organizer, DIVERSE Bird's of a Feather (BOF), SIGGRAPH 2009, New Orleans, LA.

10. V. Marbukh, Member, Technical Program Committee, Advances in Learning for Networks, ACM SIGMETRICS, Seattle, WA, June 15, 2009.

11. Y. Parker and J. Terrill, NIST Exhibit and Demonstration Booth, Supercomputing 2008, Austin, TX, November 15-21, 2008.

12. Y. Parker, J. Terrill and J. Hagedorn, NIST Exhibit and Demonstration Booth, Supercomputing 2009, Portland, OR, November 16-20, 2009.

\section{Other Professional Activities}

\section{Internal}

1. I. Beichl, Member, NIST Research Advisory Committee.

2. R. Boisvert, Member, NIST Scientific Computing Steering Group.

3. R. Boisvert, Member, ITL Diversity Committee.

4. R. Kacker, Member, NIST Library Advisory Committee.

5. W. Mitchell, Member, ITL Awards Committee.

6. A. O'Gallagher, Member, ITL Diversity Committee.

7. A. Peskin, Member, Boulder Research Advisory Council.

\section{External}

\section{Editorial}

1. I. Beichl, Editor-in-Chief, IEEE Computing in Science \& Engineering.

2. R. Boisvert, Associate Editor, ACM Transactions on Mathematical Software.

3. R. Boisvert, Editor, Numerical Analysis, Mathematical Software, and Computational Engineering, Finance, and Science areas of the Computing Research Repository (CoRR) preprint service hosted by www.arXiv.org.
4. R. Boisvert, Member, Editorial Committee, Special Issue on Innovative and Collaborative Problem Solving Environments in Distributed Resources, Journal of Convergence Information Technology.

5. D. Lozier, Member, Editorial Board, Journal of Numerical Analysis, Industrial and Applied Mathematics.

6. G. McFadden, Associate Editor, Journal of Crystal Growth.

7. G. McFadden, Associate Editor, Interfaces and Free Boundaries.

8. W. Mitchell, Associate Editor, International Journal of Applied Mathematics and Computational Science.

9. W. Mitchell, Associate Editor, Journal of Numerical Analysis, Industrial and Applied Mathematics.

10. D. Porter, Editor, November 2009 issue of IEEE Transactions on Magnetics (Selected papers from the 2009 Hysteresis and Micromagnetic Modeling Symposium).

\section{Boards and Committees}

1. I. Beichl, Member, Electronic Publications and Services Committee, IEEE Computer Society.

2. I. Beichl, Member, IEEE Magazine Operations Committee.

3. R. Boisvert, Chair, International Federation for Information Processing's (IFIP) Working Group 2.5 on Numerical Software.

4. R. Boisvert, Co-chair, Publication Board, Association for Computing Machinery (ACM).

5. R. Boisvert, Ex-Officio Member, ACM Council.

6. R. Boisvert, Member, ACM Awards Committee.

7. R. Boisvert, Member, Program Review Committee, Center for Computing Sciences, Institute for Defense Analysis.

8. R. Boisvert, Member, External Review Committee, Computer Science Department, George Washington University.

9. R. Boisvert, Member, Ad-hoc External Review Panel, Faculty of Engineering, University of Palermo (Italy).

10. F. Hunt, Member, MAA Carriage House Program Committee.

11. F. Hunt, Member, Advisory Board, Georgetown University's Masters Program. 
12. F. Hunt, Emeritus Member, Board of Trustees, Bryn Mawr College.

13. D. Lozier, Secretary, Activity Group on Orthogonal Polynomials and Special Functions, Society for Industrial and Applied Mathematics (SIAM).

14. B. Miller, Member, Math Working Group, World Wide Web Consortium (W3C).

15. D. Porter, Member, Tcl Core Team.

16. B. Saunders, Member, Selection Committee, Etta Z. Falconer Lecture, Mathfest 2009.

17. J. Terrill, Member, Federal High End Computing Implementation Task Force.

18. J. Terrill, High End Computing Research and Development, and Infrastructure Interagency Working Groups, NITRD.

\section{Reviewing}

1. Division staff members referee manuscripts for a wide variety of journals and conferences including ACM Transactions on Mathematical Software; Communications in Nonlinear Science and $\mathrm{Nu}$ merical Simulations, Computing in Science and Engineering, European Physics Letters, IEEE Symposium on Information Theory, IEEE Transactions on Magnetics, International Symposium on Information Theory, Inverse Problems, Journal of Applied Physics, Journal of Computing and Information Science in Engineering, Journal of Materials in Civil Engineering, Journal of Physics A, Mathematical Reviews, Nature, Measurement Science and Technology, New Journal of Physics, Optical Engineering, Optics Communications, Physics Letters A), Physical Review A, Physical Review E, Physical Review Letters, Quantum Information and Computation, Science, SIAM Journal on Matrix Analysis and Applications, SIAM Journal on Scientific Computing.

2. MCSD staff reviewed proposals for the following agencies/competitions during FY 2009: Binational Science Foundation, Defense Threat Reduction Agency, Department of Energy, Louisiana Faculty Pilot Funding Program, National Science Foundation, NIST ARRA Measurement Science and Engineering Grants Program, NIST Technology Innovation Program, National Science and Engineering Council of Canada, NIST Construction Grant Program, U.S.-Israel Binational Science Foundation

\section{External Contacts}

MCSD staff members make contact with a wide variety of organizations in the course of their work. Examples of these follow.

\section{Industrial Labs}

Avia Training

Boehringer-Ingelheim Pharmaceuticals

ISI Foundation (Italy)

Northrop-Grumman

Numerical Algorithms Group (NAG), Ltd. (UK)

NVIDIA

Orbital Sciences Corporation

Pfizer Global R\&D

RedPrairie

SGI

2Wire, Inc.

\section{Government/Non-profit Organizations}

Air Force Research Laboratory

Army Research Laboratory

Association for Computing Machinery

Centre National de la Recherche Scientifique (France)

Centrun voor Wiskunde en Informatica (Holland)

Department of Energy

Food and Drug Administration

IEEE

Instituto Superior Tecnico (Portugal)

International Institute of IT (India)

Institute for Defense Analysis

International Federation for Information Processing

Lawrence Berkeley National Laboratory

Lawrence Livermore National Laboratory

Los Alamos National Laboratory

Mathematical Association of America

NASA Goddard Space Flight Center

National Academy of Sciences

National Institutes of Health

National Physical Laboratory (UK)

National Security Agency

National Science Foundation

Naval Research Laboratory

Oak Ridge National Laboratory

Perimeter Institute for Theoretical Physics (Canada)

Sandia National Laboratory

Society for Industrial and Applied Mathematics

SP Technical Research Institute (Sweden)

World Wide Web Consortium

\section{Universities}

American University of Sharjah (UAE)

Beloit College

Boston University 
Bryn Mawr College

California Institute of Technology

Centre College (Kentucky)

City University of Hong Kong

Collège de Polytechnique (France)

Cornell University

Courant Institute of Mathematical Sciences

Dalhousie University (Canada)

Delft University of Technology (The Netherlands)

Drexel University

George Mason University

George Washington University

Georgetown University

Giessen University (Germany)

Hampton University

Hood College

Indiana University

Indian Institute of Technology (India)

Instituto Superior Tecnico (Portugal)

Iowa State University

Johannes Kepler University (Austria)

Johns Hopkins University

Louisiana State University

Loyola College (Maryland)

Macalester College

Millersville University

North Carolina State University

New Mexico State University

New York University

North Carolina State University

The Ohio State University

Penn State University

Seoul National University (Korea)

Sichuan University (China)

Stanford University

SUNY Binghampton

Technische Universität Berlin (Germany)

Tulane University

Universidad de Cantabria (Spain)

Universidad de Valladolid (Spain)

Universidade Federal do Rio Grande do Sul (Brazil)

Universität Duisberg-Essen (Germany)
University of Albertay Dundee (UK)

University of Amsterdam (The Netherlands)

University of Bristol (UK)

University of Buffalo

University of Cambridge (UK)

University of California at Berkeley

University of California at Los Angeles

University of California at San Diego

University of Catalonia (Spain)

University of Clermont-Ferrand II (France)

University of Colorado

University of Delaware

University of the District of Columbia

University of Edinburgh (UK)

University of Groningen (Germany)

University of Illinois at Carbondale

University of Iowa

University of Karlsruhe (Germany)

University of Kent (UK)

University of Leeds

University of Linköping (Sweden)

University of Manitoba

University of Maryland

University of Maryland - Baltimore County

University of Missouri - Columbia

University of Nebraska

University of Nevada at Reno

University of New Mexico

University of Palermo (Italy)

University of Puerto Rico, Bayamon

University of Sheffield (UK)

University of St. Andrews (UK)

University of Texas at Arlington

University of Utah

University of Waikato (New Zealand)

University of Washington

University of Wisconsin

University of Wisconsin-Milwaukee

Utah State University

Vrije Universiteit Amsterdam (The Netherlands) 


\section{Part V}

\section{Appendices}

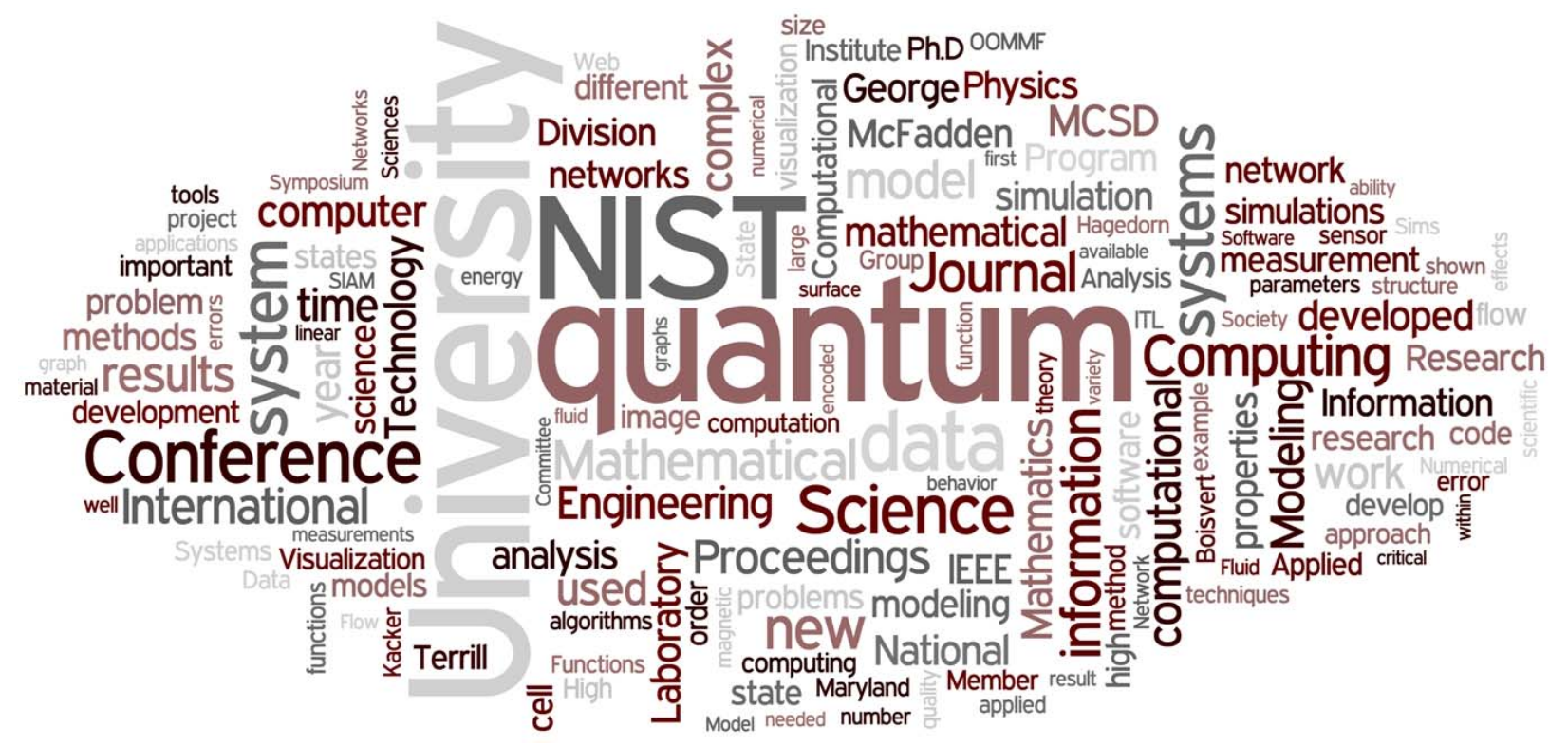




\section{$\underline{\text { Staff }}$}

MCSD consists of full time permanent staff located at NIST laboratories in Gaithersburg, MD and Boulder, CO. This is supplemented with a variety of faculty appointments, guest researchers, postdoctoral appointments, and student appointments. The following list reflects all appointments held during any portion of the reporting period. (*) Denotes staff at the NIST Boulder Laboratory.

\section{Division Staff}

Ronald Boisvert, Chief, Ph.D. (Computer Science), Purdue University, 1979

Robin Bickel, Secretary

Jeffrey Fong, Ph. D. (Applied Mechanics and Mathematics), Stanford University, 1966

Roldan Pozo, Ph.D. (Computer Science), University of Colorado at Boulder, 1991

Christopher Schanzle, B.S. (Computer Science), University of Maryland - Baltimore County, 1989

Guest Researchers

Barry Bernstein / Illinois Institute of Technology

\section{Mathematical Modeling Group}

Geoffrey McFadden, Leader, Ph.D. (Mathematics), New York University, 1979

*Bradley Alpert, Ph.D. (Computer Science), Yale University, 1990

Timothy Burns, Ph.D. (Mathematics), University of New Mexico, 1977

Alfred Carasso, Ph.D. (Mathematics), University of Wisconsin, 1968

*Andrew Dienstfrey, Ph.D. (Mathematics), New York University, 1998

Michael Donahue, Ph.D. (Mathematics), Ohio State University, 1991

Fern Hunt, Ph.D. (Mathematics), New York University, 1978

Raghu Kacker, Ph.D. (Statistics), Iowa State University, 1979

Anthony Kearsley, Ph.D. (Computational and Applied Mathematics), Rice University, 1996

Peter Ketcham. M.S. (Mathematics), University of Minnesota, 1997

Stephen Langer, Ph.D. (Physics), Cornell University, 1989

*Agnes O'Gallagher, M.S. (Applied Math), University of Colorado at Boulder, 1991

Donald Porter, Ph.D. (Electrical Engineering), Washington University, 1996

NRC Postdoctoral Associates

James Benson, Ph.D. (Mathematics), University of Missouri - Columbia, 2009

Valerie Coffman, Ph.D. (Physics), Cornell University, 2006

Aaron Lott, Ph.D. (Mathematics), University of Maryland, 2008

\section{Contractors}

Andrew C.E. Reid, Ph.D. (Physics), Queen's University, Kingston, Ontario, 1994

\section{Faculty Appointees}

Daniel Anderson / George Mason University

Dianne O’Leary / University of Maryland College Park

Florian Potra / University of Maryland Baltimore County

\section{Guest Researchers}

Mirit Aladjem / National Institutes of Health

Richard Braun / University of Delaware

David Cotrell / Lawrence Livermore National Laboratory

Gunay Dogan / Cornell University

*John Gary / NIST (retired) 
Katharine Gurski / George Washington University

Rüdiger Kessel / Germany

Sohyoung Kim / National Institutes of Health

$\mathrm{Yu}$ (Jeff) Lei / University of Texas at Arlington

Bruce Murray / SUNY Binghampton

Students

Katherine Morrison / University of Nebraska

Michael Sharpnack / NYU

\section{Mathematical Software Group}

Daniel Lozier, Leader, Ph.D. (Applied Mathematics), University of Maryland, 1979

Marjorie McClain, M.S. (Mathematics), University of Maryland College Park, 1984

Bruce Miller, Ph.D. (Physics), University of Texas at Austin, 1983

William Mitchell, Ph.D. (Computer Science), University of Illinois at Urbana-Champaign, 1988

Bert Rust, Ph.D. (Astronomy), University of Illinois at Urbana-Champaign, 1974

Bonita Saunders, PhD (Mathematics), Old Dominion University, 1985

Faculty Appointees

Frank Olver / University of Maryland College Park

G.W. Stewart / University of Maryland College Park

Abdou Youssef / George Washington University

Guest Researchers

Leonard Maximon / George Washington University

Qiming Wang / NIST (retired)

Students

Russell McFadden / St. Olaf College

Menal Modha / UMBC

\section{Discrete Mathematical Analysis Group}

Ronald Boisvert, Acting Leader

Isabel Beichl, Ph.D. (Mathematics), Cornell University, 1981

Javier Bernal, Ph.D. (Mathematics), Catholic University, 1980

Brian Cloteaux, Ph.D. (Computer Science), New Mexico State University, 2007

David Gilsinn, Ph.D. (Mathematics), Georgetown University, 1969

*Scott Glancy, Ph.D. (Physics), University of Notre Dame, 2003

*Emanuel (Manny) Knill, Ph.D., (Mathematics), University of Colorado at Boulder, 1991

Vladimir Marbukh, Ph.D. (Mathematics) Leningrad Polytechnic University, 1986

NRC Postdoctoral Associates

*Bryan Eastin, Ph.D. (Physics), University of New Mexico, 2007

Faculty Appointees

Saul Gass / University of Maryland College Park

James Lawrence / George Mason University

Guest Researchers

Stephen Bullock / IDA Center for Computing Sciences

Sita Ramamurti / Trinity University, DC

Francis Sullivan / IDA Center for Computing Sciences

Christoph Witzgall / NIST Scientist Emeritus 
Students

*Michael Mullan / University of Colorado at Boulder

*Adam Meier / University of Colorado at Boulder

Nadezhda Serova / UMBC

*Yanbao Zhang / University of Colorado at Boulder

\section{Scientific Applications and Visualization Group}

Judith Terrill, Leader, Ph.D. (Information Technology), George Mason University, 1998 Yolanda Parker, Office Manager

Robert Bohn, Ph.D. (Physical Chemistry), University of Virginia, 1991

William George, Ph.D. (Computer/Computational Science), Clemson University, 1995

Terence Griffin, B.S. (Mathematics), St. Mary's College of Maryland, 1987

John Hagedorn, M.S. (Mathematics), Rutgers University, 1980

John Kelso, M.S. (Computer Science), George Washington University, 1984

*Adele Peskin, Ph.D. (Chemical Engineering), University of Colorado at Boulder, 1985

Steven Satterfield, M.S. (Computer Science), North Carolina State University, 1975

James Sims, Ph.D. (Chemical Physics), Indiana University, 1969

Faculty Appointees

Marc Olano / University of Maryland Baltimore County

Guest Researchers

Aurelei Canale / ISIMA Campus de Clermont-Ferrand, France

Dong Yeon Cho / Seoul National University, Korea

Edith Enjolras / Université Blaise Pascal, France

Pradeep Gaddam / Core Projects and Technologies PVT LTD, India

Cedric Houard / Université Blaise Pascal Clermont Ferrand II, France

Julien Lancien / Institut National des Telecommunications, France

Audrey Lemoussu / ISIMA Campus de Clermont-Ferrand, France

Alexandre Marie / ISIMA Campus de Clermont-Ferrand, France

Jean-Loup Traore / Université Blaise Pascal Clermont Ferrand II, France

Students

Matthew Du / Thomas S. Wooton High School, Maryland

Julie Eisenberg / Damascus High School, Maryland

*Daniel Leifer / Stanford University

Sei Masouka / Winston Churchill High School, Maryland

Sanjeev Muralidharan / Montgomery Blair High School, Maryland

Joseph Owen / Montgomery Blair High School, Maryland

Jennifer Schiller / Reservoir High School

* NIST Boulder Laboratory 


\section{Glossary of Acronyms}

\begin{tabular}{|c|c|}
\hline $\mathrm{ACM}$ & Association for Computing Machinery \\
\hline ACTS & Advanced Combinatorial Test Suites (software) \\
\hline AISI & American Iron and Steele Institute \\
\hline ANSI & American National Standards Institute \\
\hline APS & American Physical Society \\
\hline ASME & American Society of Mechanical Engineers \\
\hline BAN & body area network \\
\hline BFRL & NIST Building and Fire Research Laboratory \\
\hline BLAS & Basic Linear Algebra Subprograms \\
\hline CIRP & International Academy for Production Engineering \\
\hline CiSE & Computing in Science \& Engineering \\
\hline CMM & coordinate measuring machine \\
\hline CMOS & complementary metal-oxide semiconductor \\
\hline CNST & NIST Center for Nanoscale Science and Technology \\
\hline CODATA & Committee on Data for Science and technology \\
\hline $\mathrm{CPU}$ & central processing unit \\
\hline CSTL & NIST Chemical Science and Technology Laboratory \\
\hline $\mathrm{CT}$ & computed tomography \\
\hline DARPA & DOD Defense Advanced Research Projects Agency \\
\hline DIVERSE & $\begin{array}{l}\text { Device Independent Virtual Environments - Reconfigurable, Scalable, Extensible (visualization } \\
\text { software) }\end{array}$ \\
\hline DLMF & Digital Library of Mathematical Functions \\
\hline DNA & deoxyribonucleic acid \\
\hline DOD & U.S. Department of Defense \\
\hline DOE & U.S. Department of Energy \\
\hline DPD & dissipative particle dynamics \\
\hline EEEL & NIST Electronics and Electrical Engineering Laboratory \\
\hline $\mathrm{EM}$ & electromagnetic \\
\hline $\mathrm{EM}$ & expectation maximization \\
\hline FDA & Food and Drug Administration \\
\hline FDS & Fire Dynamics Simulator \\
\hline FFT & fast Fourier transform \\
\hline FY & fiscal year \\
\hline F90gl & Fortran 90 interface to OpenGL graphics standard \\
\hline GAMS & Guide to Available Mathematical Software \\
\hline GPU & graphics processing unit \\
\hline GSTC & generalized sheet transition conditions \\
\hline GUI & graphical user interface \\
\hline HAVA & Help America Vote Act \\
\hline $\mathrm{HEC}$ & high-end computing \\
\hline HMF & Handbook of Mathematical Functions \\
\hline HTML & hypertext markup language \\
\hline Hy-CI & Hylleraas-Configuration Interaction technique \\
\hline IDA & Institute for Defense Analysis \\
\hline IEEE & Institute of Electronics and Electrical Engineers \\
\hline IFIP & International Federation for Information Processing \\
\hline IMS & Innovations in Measurement Science \\
\hline INCITE & Innovative and Novel Computational Impact on Theory and Experiment (DOE Program) \\
\hline ISO & International Standards Organization \\
\hline IT & information technology \\
\hline ITL & NIST Information Technology Laboratory \\
\hline IWG & Interagency Working Group \\
\hline JAMA & Java Matrix package \\
\hline LANL & Los Alamos National Laboratory \\
\hline
\end{tabular}




\begin{tabular}{|c|c|}
\hline LAPACK ++ & software for numerical linear algebra in $\mathrm{C}++$ \\
\hline LaTeXML & LaTeX to MathML translator \\
\hline MANET & mobile ad-hoc network \\
\hline MCSD & ITL Mathematical and Computational Sciences Division \\
\hline MEL & NIST Manufacturing Engineering Laboratory \\
\hline MICS & medical implant communication system \\
\hline MKM & mathematical knowledge management \\
\hline MMM & magnetism and magnetic materials \\
\hline MNS & minimum norm solution \\
\hline MPI & Message Passing Interface \\
\hline MRAM & magneto-resistive random access memory \\
\hline MRI & magnetic resonance imaging \\
\hline MSEL & NIST Materials Science and Engineering Laboratory \\
\hline$\mu \mathrm{mag}$ & Micromagnetics Activity Group \\
\hline NASA & National Aeronautics and Space Administration \\
\hline NBS & National Bureau of Standards \\
\hline NIH & National Institutes of Health \\
\hline NIST & National Institute of Standards and Technology \\
\hline NISTIR & NIST Internal Report \\
\hline NITRD & Networking and Information Technology Research and Development \\
\hline $\mathrm{NRC}$ & National Research Council \\
\hline NSF & National Science Foundation \\
\hline NUM & network utility maximization \\
\hline OASCR & DOE Office of Advanced Scientific Computing Research \\
\hline ODE & ordinary differential equation \\
\hline OLES & NIST Office of Law Enforcement Standards \\
\hline OOF & Object-Oriented Finite Elements \\
\hline OOMMF & Object-Oriented Micromagnetic Modeling Framework \\
\hline OSPF & open shortest path first \\
\hline PDE & partial differential equation \\
\hline PHAML & Parallel Hierarchical Adaptive Multi Level (software) \\
\hline PIMRC & Personal, Indoor and Mobile Radio Communications Symposium \\
\hline PL & NIST Physics Laboratory \\
\hline PREP & Professional Research Experience Program \\
\hline QDPD & quarternion-based dissipative particle dynamics \\
\hline QIS & quantum information science \\
\hline $\mathrm{R} \& \mathrm{D}$ & research and development \\
\hline PDE & partial differential equation \\
\hline REGEN & software for modeling of cryocoolers \\
\hline $\mathrm{RF}$ & radio frequency \\
\hline SAVG & MCSD Scientific Applications and Visualization Group \\
\hline SciDAC & Scientific Discovery Through Advanced Computing (DOE Program) \\
\hline SECB & slow evolution from the continuation boundary \\
\hline SIAM & Society for Industrial and Applied Mathematics \\
\hline SIGGRAPH & ACM Special Interest Group on Graphics \\
\hline SIMA & NIST Systems Integration for Manufacturing Applications Program \\
\hline SparseLib++ & software for spare linear algebra in $\mathrm{C}++$ \\
\hline Spblas & sparse basic linear algebra subprograms \\
\hline SPEC & System Performance Evaluation Corporation \\
\hline SPIE & International Society for Optical Engineering \\
\hline SOR & successive over-relaxation \\
\hline SUNY & State University of New York \\
\hline SURF & Student Undergraduate Research Fellowship \\
\hline TCP & transmission control protocol \\
\hline TNT & Template Numerical Toolkit \\
\hline TGDC & Technical Guidelines Development Committee \\
\hline UCLA & University of California at Los Angeles \\
\hline
\end{tabular}


UMBC University of Maryland Baltimore County

UMCP University of Maryland College Park

URL universal resource locator

VCCTL Virtual Cement and Concrete Testing Laboratory

VRML virtual reality modeling language

W3C World Wide Web Consortium

XML Extensible Markup Language

ZPE zero-point energy 